2018-09-19

\title{
Global Health Electives: Ethical Engagement in Building Global Health Capacity
}

\author{
De Visser, Adriena
}

De Visser, A. (2018). Global Health Electives: Ethical Engagement in Building Global Health Capacity (Unpublished master's thesis). University of Calgary, Calgary, AB. doi:10.11575/PRISM/33049 http://hdl.handle.net/1880/108696

Downloaded from PRISM Repository, University of Calgary 


\title{
UNIVERSITY OF CALGARY
}

Global Health Electives: Ethical Engagement in Building Global Health Capacity

by

Adriena De Visser

\begin{abstract}
A THESIS
SUBMITTED TO THE FACULTY OF GRADUATE STUDIES

IN PARTIAL FULFILMENT OF THE REQUIREMENTS FOR THE DEGREE OF MASTER OF SCIENCE
\end{abstract}

GRADUATE PROGRAM IN COMMUNITY HEALTH SCIENCES

CALGARY, ALBERTA

SEPTEMBER, 2018

(C)Adriena De Visser 2018 


\begin{abstract}
Increasingly, medical trainees are seeking global health electives (GHEs) as part of their medical education. However, little is known about the impact medical trainees undertaking global health electives have on host institutions, staff, local trainees, patients or communities in low-and middle-income countries. The goal of this study was to explore the relationship dynamics associated with global health electives as perceived by stakeholders at three sites in sub-Saharan Africa. More specifically, I examined stakeholder perspectives in Mwanza, Tanzania and Mbarara and Rugazi, Uganda where the University of Calgary Cumming School of Medicine (CSM) has long-standing institutional collaborations. In this case-based interpretive phenomenological study, thirty-four host stakeholders (health facility administrators, physicians, clinical officers, registrars, nurses, and community liaison members) participated in semistructured interviews and twenty-eight host stakeholders (medical students, residents, interns, and patients) participated in focus groups. Participant experiences were described using interpretive phenomenological analysis techniques. The findings revealed that although GHEs are well-established and a common experience for host stakeholders there are many indistinct aspects which are poorly understood by host stakeholders. Participants acknowledged that there are a variety of benefits they gain because of GHEs, but overall visiting medical trainees benefit the most from this unique learning opportunity. Despite reluctance to directly admit to harms, host stakeholders described significant challenges and burdens of GHEs and recommended ways in which GHEs could be improved to ensure that the relationships they depend on are mutually beneficial and equitable. One of the main recommendations from this study is that to work towards an equitable, effective, and ethical elective, GHEs should occur between collaborating institutions with a bidirectional focus.
\end{abstract}




\section{Acknowledgements}

This work was supported by a grant from the McLaughlin Travelling Medical Education Fund and the Canadian Queen Elizabeth II Diamond Jubilee Scholarship.

Throughout the course of my thesis I received tremendous support from a team of supervisors, advisors, mentors, colleagues, friends, and family. My utmost thankfulness to all of them for being there for me throughout this journey.

I would like to express my profound gratitude to my supervisor and mentor, Dr. Gwendolyn Hollaar. Without her guidance, encouragement, persistent help, and patience this thesis would not have been possible.

I would also like to thank Dr. Jennifer Hatfield for serving as my co-supervisor and for her valuable comments and suggestions.

Dr. Rachel Ellaway and Dr. Denise Buchner provided insights, comments, and suggestions throughout this process and I am grateful for your time, efforts, and thoughtful consideration of this work.

I was privileged to work with incredible people in Tanzania and Uganda—Dr. Jeremiah Seni, Dr. Wilfred Arubaku, Dr. Data Santorino, Josephine Najjuma, Flora Madaha, Joyce Silas, Neema Gambishi, Roze Kyarisiima, Rogers Ankunda, and Judith Owokuhaisa were instrumental in the field work and data collection process. This study would not have been possible without their assistance and I greatly appreciate their insights into local culture and context in Tanzania and Uganda.

I would like to express my sincere appreciation to the participants in this study who thoughtfully and sincerely shared their experiences. 
Finally, I would like to thank my husband, Jae, and the rest of my family for their love, support, and interest in my endeavours. They have been a blessing in so many ways and this would not have been possible without them. 


\section{Table of Contents}

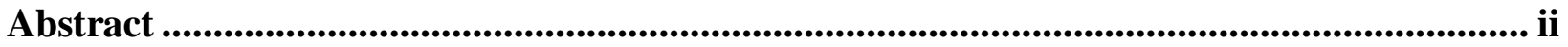

Acknowledgements................................................................................................................... iii

List of Tables and Figures.......................................................................................................... vii

Epigraph ............................................................................................................................................ viii

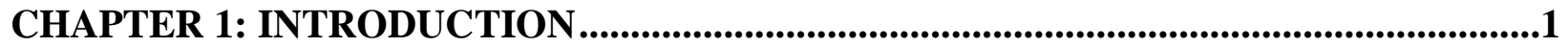

Global Health Electives.......................................................................................................................1

Cumming School of Medicine global health elective program..................................................2

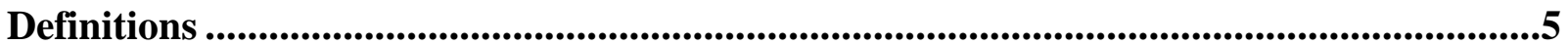

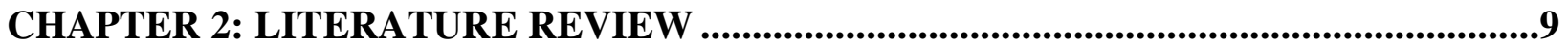

Search Strategy One: Impacts of Global Health Electives .......................................................9

Search Strategy Two: Host Stakeholder Perspectives..............................................................12

Literature Gap Regarding Host Perspectives .........................................................................19

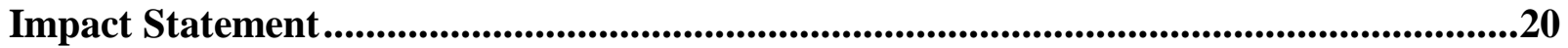

Research Question ......................................................................................................................21

CHAPTER 3: METHODOLOGY AND RESEARCH METHODS ...................................22

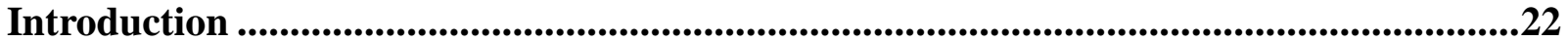

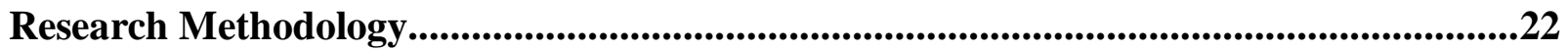

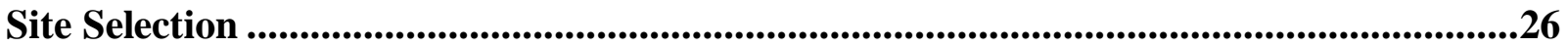

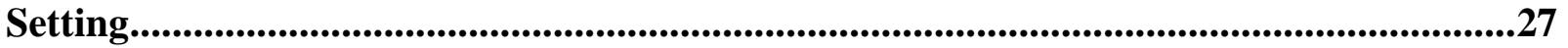

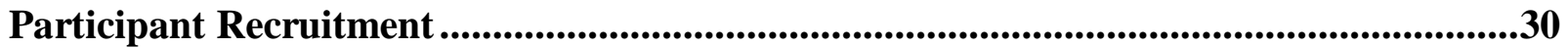

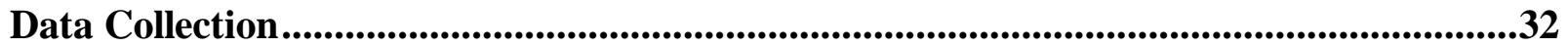

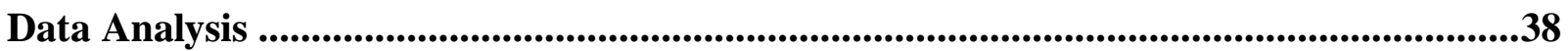

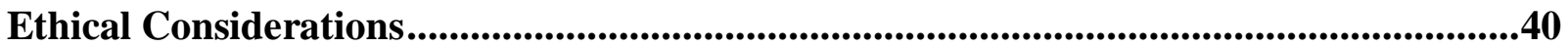

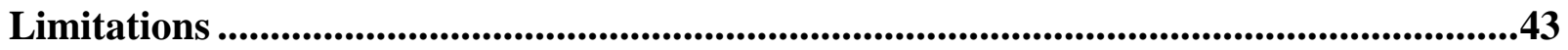

Rigour...........................................................................................................................45

Bracketing and Reflexivity ................................................................................................50

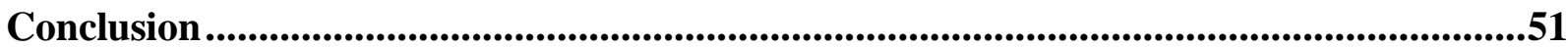

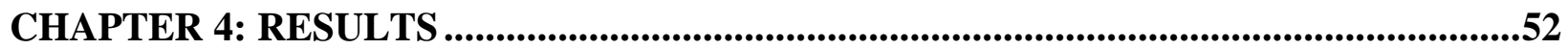

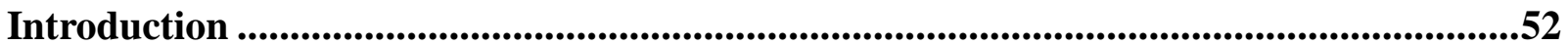

Demographics..............................................................................................................................52

Host Stakeholder Experiences with VMTs: GHEs are a common, yet indistinct phenomenon ..............................................................................................................................54

Challenges and Burdens of Global Health Electives ..................................................................77 
Recommendations for Improvement......................................................................95

Summary .....................................................................................................113

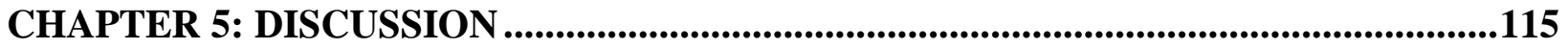

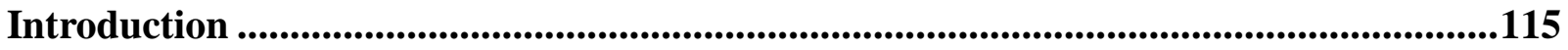

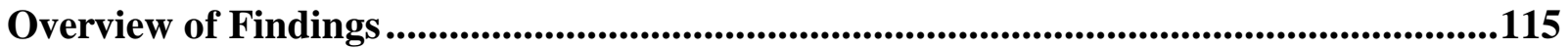

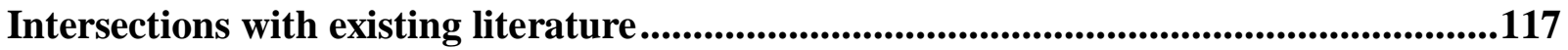

Critical appraisal of findings and how they might translate to practice.........................132

Implications for research...............................................................................................146

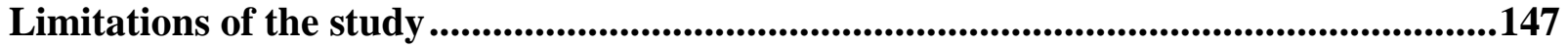

Conclusion ...............................................................................................................................149

References...................................................................................................................151

Appendix A: Guiding Principles for Funding Approval for Global Health Electives.....157 Appendix B: Focus Group Discussion Guide for University of Calgary Medical Students

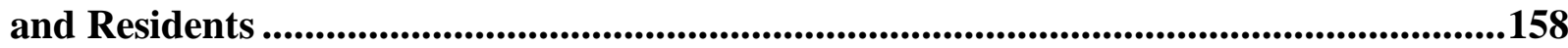

Appendix C: Interview Guide Introduction.......................................................160

Appendix C1: Interview Guide for Host Physician Preceptors ....................................161

Appendix C2: Interview Guide for Hospital/Health Facility Administrator...................163

Appendix C3: Interview Guide for Community Liaison Member....................................165

Appendix C4: Interview Guide for Nurses ............................................................166

Appendix D: Focus Group Guide Generic Introduction/Ground Rules.........................168

Appendix D1: Focus Group Discussion Guide for Medical Officers/Registrars .............169

Appendix D2: Focus Group Interview Guide for Local Residents, Interns, and Medical Students...............................................................................................................171

Appendix D3: Focus Group Discussion Guide for Patients and Family Members .........173 


\section{List of Tables and Figures}

Table 1 Number of Cumming School of Medicine medical trainees participating in global health

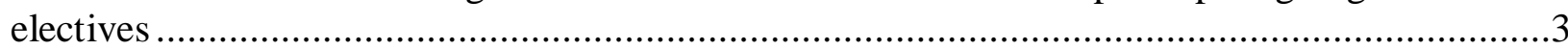

Table 2 Number of Interviews and Focus Groups Conducted at each Host Site ...........................32

Table 3 Demographics of Host Stakeholder Participants in Semi-Structured Interviews ..............53

Table 4 Demographics of Host Stakeholder Participants in Focus Groups ...................................53

Table 5 Recommendations and Practical Suggestions for Sending and Host Institutions...........139

Table 6 Recommendations and Practical Suggestions for Visiting Medical Trainees ................143

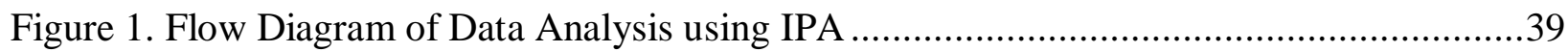

Figure 2. List of Superordinate and Emergent Themes ..........................................................116 


\section{Epigraph}

Nalukolekejaga sonda (ng’weli) walola lwala. (Sukuma)

Nilikuonyesha nyota (mwezi) na uliangalia kidole tu. (Swahili)

I pointed out to you the stars and the moon and all you saw was the tip of my finger. (English)

Tanzanian Proverb, African Proverbs, Sayings, and Stories 


\section{CHAPTER 1: INTRODUCTION}

\section{Global Health Electives}

The University of Calgary has made a commitment to creating authentic learning experiences for students, thereby preparing students to critically examine the world around them, generate new knowledge, and be part of solving societal problems. (University of Calgary, 2016) As part of the Eyes High University of Calgary International Strategy one of the targets is to have $50 \%$ of students go on an international experience before they graduate. (University of Calgary, 2013) Since the University seeks to promote meaningful international experiences for its students as part of their program of study, I argue that we need to ensure that these experiences are respectful and ethical towards host institutions.

In recent years, there has been growing medical student interest from high-income countries (HIC) in undertaking global health electives ${ }^{1}$ in low-and middle-income countries (LMIC). (Anderson et al., 2012) This has occurred within the context of growing globalization in society as a whole. (Evert, 2008) This trend is particularly evident and well-described among American and Canadian medical students, but also among residents, health science students, graduate students, and faculty. (Drain, Holmes, Skeff, Hall, \& Gardner, 2009; Liu, Zhang, Liu, \& Wang, 2015) Over time, increasing attention has been given to prepare learners for global health electives. For instance, the Liaison Committee on Medical Education (LCME), the accrediting body for medical education programs in the United States and Canada, recently required medical schools to properly prepare students that are undertaking global health experiences. (Liaison Committee on Medical Education (LCME), 2017; Purkey \& Hollaar, 2016)

\footnotetext{
${ }^{1}$ Global health elective - a clinical care or health research experience by a medical trainee from a highincome country within an underserved or resource-constrained population.
} 
Less attention has been paid to the impact of these global health electives on host institutions and host communities, whether they are causing harms or benefits. We need to better understand how global health electives are operated and the impact they have, both for practical and ethical reasons. For instance, we need to ensure that these global health electives do not perpetuate the paternalistic attitudes of colonialism and the historical legacy of unequal medical relationships between the Global North and South. (Hanson, Harms, \& Plamondon, 2011) It has been suggested that current global health electives will do little to address historically and politically rooted global health inequities unless critical consciousness is raised through improved global health curricula. (Hanson et al., 2011)

\section{Cumming School of Medicine global health elective program}

The Cumming School of Medicine at the University of Calgary (U of C) encourages its medical students, clinical clerks ${ }^{2}$, and medical residents ${ }^{3}$ to pursue global health electives. Between years one and two of the three-year MD program, $\mathrm{U}$ of $\mathrm{C}$ medical students have the opportunity to spend a month in a developing country to investigate and observe how medical care is delivered in resource-limited environments often with vulnerable or underserved populations. This opportunity (minimum of four weeks) is also available during clinical clerkship $^{2}$ and medical residency ${ }^{3}$ (minimum of two weeks). There is not a specified maximum time limit but global health electives typically range from four to six weeks as medical students and residents are limited by core academic requirements which need to be completed at the $\mathrm{U}$ of C. The following table illustrates the number of trainees participating in this program from

\footnotetext{
${ }^{2}$ Clinical clerk - a medical student who has completed two years of medical school and begins clerkship experiences in different areas of medical specialization under direct supervision.

${ }^{3}$ Medical resident - has completed a MD degree and works under direct supervision in a particular specialty of medicine for 2 to 6 years.
} 
2013-2016, with specific numbers also provided for Uganda and Tanzania, the two countries where this study was conducted.

Table 1 Number of Cumming School of Medicine medical trainees participating in global health electives

\begin{tabular}{|l|l|l|l|l|l|l|}
\hline Year & \multicolumn{2}{l|}{$\begin{array}{l}\text { Total (all LMIC } \\
\text { countries) }\end{array}$} & \multicolumn{2}{l|}{ Tanzania } & \multicolumn{2}{l|}{} \\
& $\begin{array}{l}\text { Medical } \\
\text { Student }\end{array}$ & Resident & $\begin{array}{l}\text { Medical } \\
\text { Student }\end{array}$ & Resident & $\begin{array}{l}\text { Medical } \\
\text { Student }\end{array}$ & Resident \\
\hline 2013 & 55 & 13 & 5 & 2 & 3 & 2 \\
\hline 2014 & 44 & 27 & 9 & 2 & 5 & 0 \\
\hline 2015 & 44 & 25 & 7 & 1 & 5 & 1 \\
\hline 2016 & 27 & 40 & 7 & 0 & 4 & 2 \\
\hline Total & 170 & 105 & 28 & 5 & 17 & 5 \\
\hline
\end{tabular}

For more than 12 years, the Cumming School of Medicine (CSM) has provided partial funding of $\$ 2500$ per trainee to support medical trainees to gain international medical experience. Typically, at the undergraduate level these are "medical observership" postings that do not permit students to be involved in unsupervised hands-on medical care or procedures. Medical students in these postings may observe a host physician, conduct history and physical exams with patients, and some may undertake a small research project. Resident postings involve more hands-on care and assisting with procedures. To be accepted for a global health elective a medical trainee must fill in an application form, provide a list of goals and objectives 
for the experience, and provide written proof of acceptance by an international medical professional who will act as the supervisor. These applications are approved by the Student Electives Selection Committee (a committee of faculty and a student representative). They must meet guiding principles for a global health elective that are laid out by the University of Calgary Global Health and International Partnerships Office (see Appendix A). For example, requirements include working in an underserved setting with good supervision, a safe environment, and there must be educational value for students with clearly identified learning objectives. Selected students are required to attend a faculty-led pre-departure training session that covers issues such as health and personal safety, ethical conduct, and professionalism during global health electives. Medical trainees are required to visit a travel clinic for consultation in advance of the GHE and are required to take prescribed travel medicines and vaccinations. They are also required to register with the University's Risk Management and Insurance department and Global Affairs Canada. At the time of the study, the U of C offered preceptor-led elective opportunities (where a $\mathrm{U}$ of $\mathrm{C}$ faculty member accompanies a group of medical students) in Tanzania, Uganda, Guyana, and Nicaragua but medical trainees are also encouraged to organize their own elective experiences. Over the past several years $\mathrm{U}$ of $\mathrm{C}$ medical trainees have visited more than 30 countries including countries in Africa, Asia, Eastern Europe, as well as Central and South America. Upon their return, students submit an evaluation of their global health elective along with an assignment reflecting on their experiences. The host physician preceptor must also complete an assessment of the medical trainee. 


\section{Definitions}

In the literature, the term 'global health elective' is used interchangeably with other terms such as international health experiences, international medical experiences or electives, international fieldwork placements, or short-term experiences in global health. The definition of 'global health' is still under debate with many researchers attempting to define it. (Beaglehole \& Bonita, 2010; Kickbusch, 2006; Koplan et al., 2009) It has been suggested that without a common definition of global health, important differences in philosophy, strategies, and priorities for action among physicians, researchers, funders, media, and even the general public will remain unclear. (Koplan et al., 2009)

There are different definitions of global health that focus on issues such as collaboration, shared international agendas, and calls for action. (Beaglehole \& Bonita, 2010; Kickbusch, 2006; Koplan et al., 2009) For this study, I have used the definition of global health by Koplan et al. (2009): “[an] area of study, research, and practice that places a priority on improving health and achieving equity in health for all people worldwide”, as it most closely aligns with my interest in developing global health electives which are equitable and mutually beneficial.

I have used the term 'global health elective' (GHE) to describe an international clinical care or health research experience by any visiting medical trainee from a high-income country within an underserved or resource-constrained population. (University of Calgary, 2018)

Social accountability for medical schools was defined by the World Health Organization in 1995 as, "The obligation to direct...education, research and service activities towards addressing the priority health concerns of the community, region and/or nation they have a mandate to serve. The priority health concerns are to be defined jointly by governments, healthcare organizations, health professionals and the public”. (World Health Organization, 2008) Ritz et al. (2014) described how the concept of social accountability for medical schools 
has become increasingly ambiguous in its meaning and intent. Wallace and Webb (2014) echo this concern in arguing that pre-departure training by medical schools is not enough to make global health electives socially accountable and that pre-departure training should be embedded within a focus on the larger principles of social accountability. According to the Global Consensus for the Social Accountability of Medical Schools (GCSA), socially accountable medical institutions must focus on larger principles of social accountability within global health elective programs through developing bilateral community partnerships and respecting communities as primary stakeholders in the training of medical trainees and in program evaluation. (Global Consensus for Social Accountability of Medical Schools, 2010) Therefore, there is a need to be critically reflexive when the term social accountability is used, to ensure that in the endeavor to address health inequities, local communities are engaged and involved in the dialogue, so that in serving the marginalized and disadvantaged, medical schools are not simply re-instantiating the forms of dominance that initially created the inequities. (Ritz et al., 2014) Both Ritz et al. (2014) and Wallace et al. (2014) advocate for efforts to develop a critically reflexive social accountability as a force for addressing health inequities, and one way to do this is through further studies of the community perspective on global health elective programs. In this study, I use the concept of social accountability of a medical school as: the practice of engaging with and being held to account by the communities the medical school has a mandate to serve. (Boelen \& Woollard, 2011)

Anticipating that certain participants, such as nurses and patients at the host sites, may not be able to distinguish or recall the training level of the visiting medical student or resident, I used the term 'visiting medical trainee' (VMT) to refer to all medical students and residents from 
outside the African continent, at any stage in their training who complete a global health elective there.

Low-and middle-income countries are defined by the World Bank classification system according to Gross National Income (GNI) per capita as low-income, lower-middle income, or upper-middle income countries. Both Tanzania and Uganda are classified as low-income countries by the World Bank. Another term commonly used in the global health literature in regards to global health electives is 'Western' in reference to Western medical schools or medical trainees from Western countries. However, 'Western' is an arbitrary term given that medical trainees from North America, Europe, Australia and other high-income countries participate in global health electives. Therefore, I have used the term high-income country (HIC) as also defined by the World Bank based on GNI per capita. (The World Bank)

I have used the concept of 'ethical engagement' in reference to the four main principles of ethics; autonomy or respect of persons, beneficence, non-maleficence, and justice. (Hebert, 2008) Arguably, engagement with host communities in LMIC through global health electives should be developed on these four principles. (Dwyer, 2003, 2005) Although global health elective programs are intended to be developed on the basis of justice, beneficence, and the duty to assist, there is scant literature, based on my literature review described in Chapter Two, documenting that this is actually occurring from the perspective of host stakeholders. (Crump \& Sugarman, 2008; Macfarlane, Jacobs, \& Kaaya, 2008) Crump and Sugarman (2008) argued that global health electives should be reframed to accommodate mutual and reciprocal benefit through ethical engagement by engaging host stakeholder perspectives.

Finally, my use of the term 'relationship dynamics' in my research question relates to understanding host stakeholder perspectives on their relationship with VMT global health 
electives with regards to harms, benefits and reciprocity. The Oxford Dictionary defines dynamics as "the forces or properties that stimulate growth, development, or change within a system or process: 'the dynamics of changing social relations' ”. (Oxford English Dictionary Online) This definition highlights that global health electives are a 'force' that impacts host stakeholders, something I investigate in this study. 


\section{CHAPTER 2: LITERATURE REVIEW}

In my literature review of global health electives, I did a two-step review focusing on two topics related to my project: impacts of global health electives including benefits and drawbacks, and host perspectives on global health electives. Within this chapter, I describe the search strategies and selection process of available literature regarding global health electives. Furthermore, I provide a critical appraisal of the selected literature on host stakeholder perspectives regarding global health electives and a summary of the evidence.

\section{Search Strategy One: Impacts of Global Health Electives}

I conducted an exploratory review of the literature, without using a systematic methodology, for articles related to the impacts of global health electives. My rationale for this exploratory review was to gain a general overview of the global health literature and find specific articles related to impacts of global health electives. I searched two major electronic databases, Medical Literature Analysis and Retrieval System Online (MEDLINE) and Cumulative Index to Nursing and Allied Health Literature (CINAHL) with the combination of keywords (global health* OR International Educational Exchange OR international health experience* OR global health elective* OR international medical elective*) AND (education* OR training* OR experience* OR elective*). A substantial body of literature regarding global health education or electives was identified. To obtain a broad overview of the impacts of global health electives I did a full-text review of four systematic reviews which summarized a significant amount of evidence on global health electives. For specific details on impacts, I used the review articles' reference lists to identify key articles which reported primary research on the 
impacts of global health electives.

\section{Impacts of Global Health Electives.}

I found four reviews that summarize a substantial body of literature describing educational effects of global health electives, strategies for global health training, and gaps in studies of global health education for high-income country (HIC) medical trainees. (Liu et al., 2015; Mutchnick, Moyer, \& Stern, 2003; Thompson, Huntington, Hunt, Pinsky, \& Brodie, 2003b; Walker, Campbell, \& Egede, 2014). These four systematic reviews were a critical review, literature search, systematic search, and narrative review respectively.

Liu et al. (2015) found that only 53 articles were published on global health education between 1987-2007, but there was a rapid increase in articles addressing global health education leading up to their review. However, the majority of studies (94.6\%) were from North American and European countries, with most focusing on needs assessment for global health training of HIC trainees or descriptions of global health programs. Very few studies were from low-and middle-income countries (LMIC) and none from Africa. This trend is troubling as much of global health education takes place in LMIC. In Walker et al.'s (2014) narrative review regarding the evidence on effective strategies for global health research, training, and clinical care, eight articles were reviewed regarding effective strategies for training. Six of the eight articles provided recommendations for preparation of students, four mentioned the importance of long-term commitments, four noted aspects used to increase impact, and three stated the need for mentorship. One of the articles does not mention visiting medical trainees (VMTs) but rather focuses on NGOs; however, the remaining seven articles all focus on HIC trainees and HIC global health training programs. Mutchnick et al. (2003) conducted a literature review to 
evaluate the evidence for cross-cultural exchanges and found 42 relevant studies within the nursing and medical literature. The authors reported that all studies identified positive outcomes along four domains: trainees’ professional development, trainees’ personal development, medical school benefits, and host population benefits. Only four out of 42 studies reported on host population benefits. However, as briefly mentioned by the authors, it is concerning that none of these 42 studies reported on the potential negative effects of cross-cultural experiences. Thompson et al. (2003b) performed a literature review to evaluate the educational effects of global health electives on participants and found eight relevant studies involving 522 medical students and 166 residents. Again, none of the studies in this review reported on challenges of global health electives (GHEs) or negative effects for visiting medical trainees. This may reflect that self-reported questionnaires were not designed to assess challenges or potential negative effects. Furthermore, Thompson et al. (2003b) did not identify any studies that reviewed effects of GHEs on patients or host communities.

Benefits mentioned by medical trainees in self-reported questionnaires are increased knowledge (e.g., tropical diseases, cross-cultural issues, public health, healthcare delivery) and enhanced skills (e.g., problem-solving, clinical examination, laboratory expertise, and language). (Abedini, Gruppen, Kolars, \& Kumagai, 2012; Holmes, Zayas, \& Koyfman, 2012; Thompson et al., 2003b) Multiple studies reported that medical trainees who participated in global health electives were more likely to care for underserved populations and become involved in primary care. (Godkin \& Savageau, 2003; Mutchnick et al., 2003; Thompson, Huntington, Hunt, Pinsky, \& Brodie, 2003a) These medical trainees were also more likely to be interested in humanitarianism and public health after they finished their medical program.

There is growing discussion about the ethical implications of global health electives. 
(Ackerman, 2010; Crump \& Sugarman, 2008; Pinto \& Upshur, 2009; Shah \& Wu, 2008b) Some of the ethical concerns include health risks to VMTs, negative impacts on local patients due to language and cultural barriers, medical trainees practicing beyond their training, increased time demands on host preceptors, and financial burdens to host institutions. (Crump \& Sugarman, 2008; Dell, Varpio, Petrosoniak, Gajaria, \& McCarthy, 2014; Shah \& Wu, 2008b) Studies that report on implications of global health electives derive primarily from perspectives of HIC medical trainees and sending institutions. For example, Shah and Wu (2008b) wrote an editorial based on their personal experiences working with medical students who participated in GHEs and provided one vignette as an example of an ethical challenge and negative outcome. Elit et al. (2011) conducted semi-structured interviews with twelve medical students who completed a GHE and found five themes related to ethical issues. As illustrated by these articles, rarely does the data focus on the host country perspectives.

A set of ethical guidelines for global health programs were drafted in 2010 by a Working Group on Ethics Guidelines for Global Health Training (WEIGHT) (Crump, Sugarman, \& the Working Group on Ethics Guidelines for Global Health Training, 2010) drawing on thirteen individuals involved in global health from five American universities, the National Institutes of Health, the London School of Hygiene and Tropical Medicine, and universities and medical centers from India, Tanzania, Peru, Thailand, and Uganda. One of the WEIGHT recommendations included a call for a more formal assessment of the benefits and harms to institutions, staff, trainees, and communities in host countries.

\section{Search Strategy Two: Host Stakeholder Perspectives}

In addition to my exploratory review, literature focusing on host stakeholder perspectives 
of global health electives was identified through two major electronic databases, MEDLINE and CINAHL. I searched MEDLINE on February 2, 2017, using Boolean search techniques with the combination of keywords of (global health* OR International Educational Exchange OR international health experience* OR global health elective* OR international medical elective*) AND (education* OR training* OR experience* OR elective*) AND (qualitative* OR mixed methods*), without year or language restrictions. 209 records were retrieved, and once duplicates were removed, 200 records remained. I reviewed these titles and abstracts to identify articles that discussed host perspectives resulting in 19 articles for full-text review. Final articles that were considered eligible all included primary research on host community perspectives of medical trainee’ and allied health professional trainee’ global health electives in a low-andmiddle income country. Four articles met the inclusion criteria. Examples of excluded articles were those which only assessed HIC trainees’ perceptions, those which looked at the impact of global health research projects on LMIC, and impact of fully-trained healthcare professionals’ medical volunteer work. CINAHL was searched on April 17, 2017, using the same search terms as in MEDLINE with 1088 records retrieved. Three additional articles were identified which examined host stakeholder’ perspectives.

I updated the search on March 14, 2018. 26 new records were retrieved in MEDLINE with no duplicates. 275 records were retrieved from CINAHL and one duplicate was removed. Reviewed titles and abstracts resulted in 5 articles for full-text review. Two additional articles were identified which examined host stakeholder' perspectives. One additional article was added from the review of reference lists of selected articles on host stakeholder perspectives. 


\section{Host Stakeholder Perspectives.}

I found five articles that evaluated host stakeholder perceptions on medical trainee’ global health electives (Bozinoff et al., 2014; DeCamp, Enumah, O'Neill, \& Sugarman, 2014; Kumwenda, Dowell, Daniels, \& Merrylees, 2015; Kung et al., 2016; Lukolyo et al., 2016). Two of the articles described host stakeholder perceptions on nursing students' global health electives (Smit \& Tremethick, 2014; Underwood, Gleeson, Konnert, Wong, \& Valerio, 2016), one evaluated host perspectives on occupational therapy student' global health electives (Simonelis, Njelesani, Novak, Kuzma, \& Cameron, 2011), and one evaluated host perspectives on physical therapy student' global health electives. (Hayward \& Li, 2017) One other article evaluated host stakeholder perceptions of health professional student volunteers, but the authors did not identify in which undergraduate disciplines the students were enrolled. (Evans et al., 2017) While these studies provided some insights into host stakeholder perspectives, not all stakeholders were represented, and there was an absence of in-depth data.

\section{Host Stakeholder Perspectives on Medical Trainee’ Global Health Electives.}

Five articles reported on host stakeholder perspectives in regards to medical trainee’ global health electives. Bozinoff et al. (2014) analyzed 17 self-reported questionnaires from different host physician preceptors about medical student' global health electives and identified themes related to: 1) the perception of mutual benefits, 2) a consideration of negative impacts, and 3) suggestions for future collaboration. A key limitation of this work, as mentioned by the authors, was that the study only assessed the perspective of host physician preceptors, which may not have reflected the perspectives of other staff, patients or local trainees in the host community. Similarly, Lukolyo et al. (2016) reported on a survey of 32 host physician preceptors in subSaharan Africa. Concerns regarding visiting medical trainees’ professionalism, teamwork, and 
knowledge gaps in understanding differences in health systems and epidemiology were raised in participant's responses to open-ended questions. This is despite $97 \%$ of participants stating they are satisfied in their role hosting VMTs. It is difficult to understand how host physician preceptors feel satisfied as this data is quantitative whereas the authors explored the concerns qualitatively. Both these studies used questionnaires or surveys which do not allow for the indepth exploration of perspectives that semi-structured interviews provide. Kumwenda et al. (2015) conducted 14 semi-structured interviews with host physician preceptors at seven sites in Malawi, Zambia, and Tanzania. This study concluded that electives would benefit from better organization and planning and that Western medical schools should actively consider the development of clear frameworks, including formal partnerships, with institutions hosting visiting medical trainees in resource-poor areas. However, as before, this study does not provide a holistic perspective of all stakeholders at each host site.

A greater variety of stakeholders were included in Kung et al.'s (2016) study based on 35 semi-structured interviews in Bolivia and India, with host physicians, local NGO directors, social workers, program administrators, and home-stay family members who provided accommodation for the visiting medical trainees. Their results indicated that benefits for the host stakeholders interviewed in their study included improvements in job satisfaction, local prestige, global connectedness, local networks between medical directors and community-based physician educators, leadership skills, resources and sense of efficacy within their communities. Participants felt that improvements should be made in high-income country trainee attitudes and behaviours and asked that visiting medical trainees not make promises they would not fulfill. Another drawback reported was host physician preceptor frustration with lack of parity in professional development opportunities. It is unclear what the authors mean by 'HIC trainees' 
because they do not define this term. In their introduction, they discussed medical student involvement in GHEs but based on their description of the study setting it appeared that they evaluated host perspectives on all undergraduate, graduate, and post-graduate HIC interprofessional trainees. Furthermore, interviews conducted in India were only with those participants who were fluent in English and therefore may not have been representative of the community.

DeCamp et al. (2014) conducted semi-structured interviews with care recipients of a short-term medical outreach program in the Dominican Republic. Since this outreach program involved visiting trainees, including nursing students, medical students, or other undergraduate students, the authors asked participants how they perceived the involvement of trainees in the outreach program. Participants did not express any negative views regarding trainees and described trainee involvement in three ways: as beneficial to the student's professional development, as beneficial to the communities because of the care delivered, and as part of future non-specific benefits to the community. A limitation of this study is that it is unclear whether the authors sought to gain perspectives regarding a variety of student roles within the outreach program including general helpers, translators, medical students, and nursing students or whether they only focused on perspectives related to medical students. Unique differences between student role's and possible differential impact on the community were not discussed by the participants or the authors. Furthermore, the interviews were conducted by the foreign investigator which may have led to significant courtesy bias, a limitation not discussed by the authors. Courtesy bias is a tendency for participants to understate their dissatisfaction as they do not want to offend the individual or organization seeking their opinion. (Green \& Thorogood, 2014) 
While the five studies provided insights into perspectives of some of the stakeholders at the host site regarding VMT global health electives, they did not report on the perspectives of local trainees and only one study reported on the perspectives of patients. Three of the five studies only engaged host physician preceptors, and only Kung et al.'s study included more than one type of stakeholder. Although all of these studies described the methodology used to analyze the data, none of them described the theoretical framework used to guide the study. All, except one, of the studies reported on challenges or drawbacks of GHEs for the host institution and host stakeholders with common challenges reported regarding VMTs’ professionalism and inadequate pre-departure preparation. All authors concluded that there are numerous benefits of GHEs with several challenges which need to be addressed.

\section{Host Stakeholder Perspectives on Allied Health Professional Trainee’ Global Health Electives.}

I found two studies in the field of nursing, one study in the field of occupational therapy, one study in the field of physiotherapy, and one study on health professional students that evaluated host perspectives on trainee’ global health electives. Smit et al. (2014) conducted structured interviews with Honduran nurse preceptors. The authors reported that Honduran nurse preceptors perceived supervising American nursing students as a positive experience with the only challenge identified being a language barrier. The main recommendation by the participants was that they would like more time with the nursing trainees. Unfortunately, although the authors were able to interview 15 Honduran nurse preceptors before their experiences with visiting nursing students, they only were able to interview three Honduran nurses after the nursing students’ global health elective. The small sample size makes it difficult to draw definitive conclusions. 
Underwood et al. (2016) conducted focus groups and semi-structured interviews with 23 Dominican stakeholders, including school teachers, health professionals, health committee laypersons, community members, NGO leaders, interpreters, and a host coordinator regarding nursing students' global health electives. Some of the key themes identified were the importance of pre-departure preparation, clear communication from planning to evaluation, and a need for equitable collaboration in jointly designing, implementing, and evaluating placements and health initiatives. Simonelis et al. (2011) used telephone interviews to assess host perspectives of four teachers in Trinidad on occupational therapy students' electives. The main themes identified in this study were collaborative learning, cultural negotiations because of clear cultural differences, and the need for independent thinking. Although the authors mentioned that challenging experiences were reported, the perspectives related by the Trinidad teachers were overwhelmingly positive. Both Underwood et al. and Simonelis et al.'s studies may have been subject to courtesy bias, a limitation not discussed by the authors, as the foreign investigators conducted the interviews either in person or over the phone.

Hayward and Li (2017) conducted interviews with six key global partner staff and collected surveys from 25 support staff at two orphanages in Ecuador where American physical therapy students travelled for a nine-day international service learning experience. However, the surveys did not allow for capture of in-depth data. For example, the authors described that the statement in the Likert survey that received the lowest score of 'disagree' or 'strongly disagree' was "the benefits of having physical therapy student group working at the orphanage outweighed the burden of additional workload." This is an interesting finding among the support staff but was not reflected in the key stakeholder interviews and should, therefore, be further explored. The interview data may have been subject to courtesy bias as the lead author was present during 
all of the interviews.

Evans et al. (2017) conducted interviews with 21 community members in two different rural villages in the Dominican Republic regarding their perspectives on American health professions undergraduate student volunteers. The only theme reported relates to benefits due to increased physical resources for the community and entertainment or gifts provided by the student volunteers to the community. The first question in this study asked of community members was: "If the volunteers were to permanently leave today, how would the community be affected?” This question may have led participants to fear that the volunteer teams would leave if their responses were negative or neutral. It is unclear from the methods who conducted the interviews and therefore I cannot determine whether courtesy bias may be a limitation. Although one of the allied health professional studies presented data from a wide variety of stakeholders, most studies included in this review failed to provide a holistic, in-depth perspective of host stakeholder views and none of the studies discussed courtesy bias as a potential limitation. However, a strength of these studies was that they all discussed the theoretical framework used to guide the study.

\section{Literature Gap Regarding Host Perspectives}

Although these studies comprised host stakeholder perceptions of global health electives, most studies only included host supervisor perspectives. Furthermore, the majority of studies overwhelmingly report on the benefits of GHEs, yet many of the studies may have been subject to significant courtesy bias as the foreign investigator conducted the interviews. Although I found a significant amount of research on the impacts of global health electives based on research with VMTs or HIC academic professionals, very little research in comparison has 
elicited host stakeholder perspectives. Further research is warranted to ensure a robust understanding of all host stakeholder perspectives and not only those of host preceptors. This one-sidedness of perspectives within publications on global health electives is concerning as LMIC host institutions have received VMTs since at least 1968 for international educational rotations. (Waddell, 1976) The narrative review by Walker et al. (2014) recommended that global health training experiences should be meaningful, relevant, and ethical to the local community. Ethical engagement, as one of the primary goals of this study, seeks to include or engage all relevant stakeholder perspectives, to promote ethical global health electives through equitable partnerships and shared benefits for all. Through ethical engagement, this study also seeks to build global health capacity in creating equitable partnerships.

\section{Impact Statement}

My goal with this study was to address a gap in the medical education literature related to host stakeholder perspectives on visiting medical trainee' global health electives. My literature review demonstrates that the perspective of host stakeholders, including physician preceptors, nurses, medical officers, local trainees, patients, and community perspectives related to global health electives has been under-reported in the literature. Given the number of medical trainees involved in the University of Calgary global health elective program, seeking the perspectives of partner institutions on global health electives is an important ethical and socially accountable process that our academic institution needs to attend to. This qualitative study explored host stakeholder perspectives at partner institutions within Tanzania and Uganda. I undertook this study with the hope that the information and themes gained from this study might provide valuable information to the further development of the University of Calgary global health 
elective program as well as the building of mutually beneficial partnerships with host institutions and communities in Mwanza, Tanzania and Rugazi and Mbarara, Uganda. Although this study is context-bound, it provides a holistic perspective from multiple stakeholders, and there are transferable concepts within the study results to guide other HIC and LMIC institutions in setting up effective global health electives which promote better mutual gain.

\section{Research Question}

Research Question: At sites in sub-Saharan Africa where the Cumming School of Medicine (CSM) has long-standing collaborations in support of global health electives, what are the relationship dynamics as perceived by stakeholders in regards to visiting medical trainee global health electives?

\section{Specific Objectives}

I aimed to:

1)Explore and explain perspectives regarding the harms, benefits, and other impacts of global health electives from multiple stakeholders.

2)Explore and explain perspectives on how to create equitable and mutually beneficial global health electives from multiple stakeholders.

The stakeholder perspectives that I planned to include were those at the host site in Mwanza, Tanzania and Rugazi and Mbarara, Uganda: health facility administrators, host physicians, medical officers, nurses, local trainees, patients and/or family members, and community leaders. 


\section{CHAPTER 3: METHODOLOGY AND RESEARCH METHODS}

\section{Introduction}

In this chapter, I describe the underlying reasons for the choice of research methodology which reflect the study objectives. I provide an overview of site selection and sampling, the setting of the study, recruitment of study participants, and the data collection process. Within data analysis, I outline the methods used. I examine ethical considerations during the study. Prior to data collection and analysis, I anticipated some limitations, therefore, I state how this affected the research design. I discuss principles related to determining the rigour of the study based on the trustworthiness in transferability, credibility, dependability, and confirmability of the study findings and analysis.

\section{Research Methodology}

Given the complex web of issues involved in global health electives and my desire to understand the breadth of perspectives from host stakeholders, I designed the study using a qualitative methodology. My ontological framework in approaching this study was that there are multiple social realities, and I designed the study to report these multiple realities using various forms of evidence in themes, by using the actual words of different individuals and presenting different perspectives. (Creswell, 2013) Concerning the epistemological framework, I employed an interpretative approach to better understand participant' perceptions of the 'reality' of the world. (Green \& Thorogood, 2014) My data collection process involved a combination of phenomenological and case study research methods. By gaining a deeper understanding of how host stakeholders at the selected sites in Uganda and Tanzania perceive relationship dynamics 
within global health electives, in regards to harms, benefits, and equity, the study was intended to guide policies to make University of Calgary global health electives more mutually beneficial and equitable.

Interpretative phenomenological analysis (IPA) as described by Smith et al. (Smith, 2004; Smith \& Osborn, 2003) seeks to explore in detail participants’ personal lived experience and how participants make sense of that particular experience. IPA is concerned with the individual's perception or account of the event or object, rather than to produce an objective statement about the object or event itself. It is phenomenological in that it focuses on individuals' perceptions of objects or events, and it is interpretative in that one of the tenets is the analyst making sense of that personal experience. Typically, IPA uses semi-structured interviews with a small sample size to ensure rich detail in personal accounts. However, focus groups may also be used, with caution, as long as participants can discuss their own experiences with sufficient detail. Analysis of focus group discussions, therefore, require careful separation of group patterns and personal accounts. This study used both semi-structured interviews and focus groups.

I also employed case study methodology to guide this study as described by Yin. (2012) Given that our data collection occurred at three sites, two in Uganda and one in Tanzania, these host sites can be considered bounded entities (in place and time). Case study methodology has been defined as, "the desire to derive a(n) (up)close or otherwise in-depth understanding of a single or small number of "cases," set in their real-world contexts.” (Yin, 2012) This definition highlights the objectives of this study as I sought to gain a holistic, in-depth understanding of the relationship dynamics in regards to visiting medical trainee global health electives specifically at three host sites within Uganda and Tanzania. Therefore, the context of this study is essential in 
the interpretation of stakeholder perspectives, as well as the transferability of study findings. Case study methodology also uses multiple forms of evidence. This study used focus groups and semi-structured interviews as the primary form of evidence. Informal observations and field notes by the investigator were also used to inform the study results.

This is an interpretive phenomenological study within a case study inquiry. My goal was to understand the essences of the phenomenon of global health electives at three particular sites which are bounded entities in terms of place and time. Combining these two methodologies allowed me to use space triangulation as a research strategy to ensure complete and thorough findings thereby providing breadth and depth to my investigation. (Denzin \& Lincoln, 1994) This approach also revealed the varied dimensions of the phenomenon of global health electives and helped to create a more accurate description. Space triangulation consists of collecting data at more than one site and is a type of data triangulation. (Speziale \& Carpenter, 2007) One of the strengths of this strategy is it allows assessment of data convergence and divergence within different contexts that experience the same phenomenon. However, some of the weaknesses that I had to be cautious about was difficulty in dealing with vast amounts of data and false interpretation due to the overwhelming amount of data. I addressed this weakness through independent analysis of the data by research team members and discussing the emerging themes with the entire research team including the research collaborators in Uganda and Tanzania.

I combined case study methodology and interpretive phenomenology in several ways to guide my study. During the data collection phase, I documented specific details regarding the context of the study. In the data analysis phase, I analyzed the transcripts using the process described for interpretive phenomenological analysis. Once I completed the analysis, the results were compared based on sites (space triangulation), to assess for thematic similarities and 
differences. Furthermore, multiple forms of evidence as characteristic of case study

methodology were also used to triangulate themes that were identified using IPA. As Yin (2012)

describes, there is a need to constantly check and recheck the consistency of the findings from

different, as well as the same sources. I employed this method both throughout my data

collection and data analysis. Preliminary results during my data collection were reviewed with

the interview moderators and further probed in semi-structured interviews with host stakeholders

in similar or different roles. In my data analysis, I compared results across different stakeholder

roles, focus groups and interviews. Using this combination of methodologies increased the

robustness of my study.

\section{Development of Interview Guides.}

I developed interview guides through an iterative process, in consultation with all committee members, as well as LMIC research collaborators. More specifically, I created openended questions to encourage participants to describe their experiences with VMTs, while ensuring that the interview questions aligned with the research question and objectives. A review of the literature also led to development of questions regarding gaps in our understanding of host stakeholder perspectives, for example, the perceived benefits or drawbacks of a foreign preceptor accompanying VMTs. Feedback on the interview protocol was sought from all research committee members as well as LMIC research collaborators in Tanzania and Uganda. I conducted two focus groups with medical students and residents at the University of Calgary who completed a global health elective in 2017. (see Appendix B) This took place before the fieldwork in Uganda and Tanzania, and themes from these focus group discussions were used to further develop the interview guides for use at the host sites as well as reflection for myself as I 
undertook this study. However, the themes from the $\mathrm{U}$ of $\mathrm{C}$ trainee focus groups are not included in my dissertation as these focus groups were part of the methodology, but the themes did not relate to answering the objectives of the study.

\section{Site Selection}

I was interested in sites where University of Calgary (U of C) medical trainees regularly undertake global health electives and where the $\mathrm{U}$ of $\mathrm{C}$ has a long-term relationship with local providers. I selected three host sites:

1. Catholic University of Health and Allied Sciences (CUHAS)/Bugando Medical Centre, Mwanza, Tanzania

2. Mbarara University of Science and Technology (MUST)/Mbarara Regional Referral Hospital, Mbarara, Uganda

3. Rugazi Health Centre IV, Rubirizi District, Uganda (affiliated with Mbarara University of Science and Technology)

I attempted to include a fourth site in Mwanza, Tanzania, at a private medical centre; however, due to time constraints and poor response rate from invited stakeholders at this site, I only conducted one interview there. Unfortunately, the audio recording failed for this interview. Therefore, this study included only one site in Tanzania.

I had multiple reasons for selecting the three host sites. The University of Calgary Cumming School of Medicine (CSM) has long-standing institutional collaborations with both MUST and CUHAS. Leaders within the CSM's Global Health and International Partnership's office were interested in investigating these three sites in particular to improve the quality of engagement within these collaborations. In 2014, Tanzania was confirmed as a country of focus for the Government of Canada’s international development efforts with 101 million dollars being 
provided by Canada’s government to Tanzania. (Government of Canada, 2017) To align this study with national efforts was another reason for selecting a Tanzanian host site. Logistically, given time constraints and limited funding, including more sites was beyond my means for this project. The $\mathrm{U}$ of $\mathrm{C}$ collaboration with the Ugandan and Tanzanian host sites allowed for the identification of transcriptionists and interview moderators who have experience in qualitative research before travel to the host sites by myself. Given that IPA focuses on rich, detailed accounts and typically involves smaller sample sizes I felt these three sites would provide sufficient information to answer my research question and fulfill my objectives.

\section{Setting}

\section{Bugando Medical Centre, CUHAS, Mwanza, Tanzania.}

All interviews in Tanzania were conducted at Bugando Medical Centre in Mwanza, Tanzania. Tanzania is a vast country in Eastern Africa with a population of approximately 55 million people. (The World Bank, 2017) On the Human Development Index, Tanzania ranks $151^{\text {st }}$ out of 189 countries. Swahili is the official language in Tanzania, however, over 126 different languages are spoken in the country. English is the official language used in medicine, in terms of teaching as well as patient charting and medical rounds. (United Nations Development Programme, 2018) Mwanza is the second-largest city in Tanzania located on the shores of Lake Victoria with a population of approximately 700000 based on 2012 census data. (Overseas Development Institute, 2016) Bugando Medical Centre (BMC) is a 900-bed teaching, referral, and consultant Zonal (tertiary-care) Hospital located at the top of Bugando Hill in Mwanza. (Catholic University of Health and Allied Sciences, 2018) The hospital is run in partnership with the government and the Tanzania Episcopal Conference of the Catholic Bishops 
of Tanzania. CUHAS is situated within the BMC premises and began as a college in 2003, becoming a fully-fledged university in 2011. In 2016/2017 enrolment numbers for CUHAS undergraduate programs (MD, BMLS, BPharm, BScNED, BScN) were a total of 1830, diploma programs had a total of 700 students, postgraduate programs (MMED, MPH) 128 students, and Ph.D. programs had 12 students. Multiple collaborations were established between CUHAS and the $\mathrm{U}$ of $\mathrm{C}$ including the development of a Master's in Public Health program at CUHAS with the inaugural class beginning in 2010. Another collaboration between CUHAS and the U of C is to improve teaching in mental health which started in 2015.

Both medical students and residents from the $\mathrm{U}$ of $\mathrm{C}$ have participated in global health electives at BMC for more than ten years. $\mathrm{U}$ of $\mathrm{C}$ preceptor-led electives for $\mathrm{U}$ of $\mathrm{C}$ medical students began to Tanzania in 2012. Some of these preceptor-led electives have also been to Aga Khan Medical Centre which is a smaller private hospital near BMC. Based on anecdotal data, BMC receives approximately forty to fifty visiting medical trainees every year. (J.Seni, personal communication, February 17, 2018)

\section{Mbarara Regional Referral Hospital, Mbarara, Uganda.}

Within Uganda, the majority of interviews and focus groups were conducted at the Mbarara Regional Referral Hospital (MRRH), with several interviews and a focus group held at Rugazi Health Centre IV, which is a two-hour drive from Mbarara. Uganda is a land-locked country located in sub-Saharan Africa, to the northwest of Tanzania. The official language in Uganda is English although there are over thirty-two different local languages. (Simons \& Fennig, 2018) The local language spoken in the region where I conducted my research in Uganda is Runyankole. Mbarara has a population of just over 195000 as of 2014 and is the 
fifth-largest city in Uganda. (Republic of Uganda, 2018) Mbarara is approximately three to fourhour drive from the capital city of Kampala. Within Uganda, the levels of health service delivery include four levels of health centres, general hospitals, regional referral hospitals, and the national referral hospital. Health centres are designated from I-IV based on the level of services they provide, with level IV providing the greatest amount of services, including both inpatient and outpatient services, and are ranked just below general hospitals. (The Health Infrastructure Working Group, 2011) Health Centre I ranks the lowest amongst the health centres, and this would be the Village Health Teams which provide community preventive and health promotion services. Health Centre IV ideally has a target population of approximately 100000.

MRRH is a government-owned teaching and referral hospital with 600 beds. (Ministry of Health, 2018) MRRH received approximately 140 visiting medical trainees in 2017. (S. Twinamatsiko, personal communication, February 16, 2018) University of Calgary medical students who complete a global health elective at Rugazi Health Centre IV, also spend approximately one week at MRRH. Two U of C pediatrics residents completed a global health elective at MRRH in 2017.

MRRH and Mbarara University of Science and Technology (MUST) are located on the same campus. MUST began in 1989 as Uganda's second public university to support education and research in science and technology. (Mbarara University of Science and Technology, 2018) The $\mathrm{U}$ of $\mathrm{C}$ has also established various collaborations with MUST. One of the recent collaborations is the establishment of a simulation centre at MUST for teaching undergraduate medical students and postgraduate students. The second partnership is Healthy Child Uganda whose main focus is the establishment of Village Health Teams (VHTs) to improve the health of mothers and children in southwestern Uganda. Healthy Child Uganda was established in 2002. 


\section{Rugazi Health Centre IV, Rubirizi District, Uganda.}

Rugazi Health Centre IV is a sub-district hospital located approximately two-hour drive northwest of Mbarara on the edge of Queen Elizabeth National Park, a popular tourist safari destination. Although the district medical officer managing Rugazi Health Centre IV is a physician, the primary health care professional on-site is typically a clinical officer, who has two years of basic medical training. Rugazi Health Centre IV employs approximately two to three physicians although they are not present year-round. The facility is composed of multiple onestory buildings including a maternity ward, female and male medical ward, pediatric ward, one operating theatre, outpatient clinic building, HIV clinic, administration, student dormitory, and one classroom. Since 2015, a group of approximately three to four U of C medical students completed preceptor-led global health electives at this site every year. Rugazi Health Centre IV also receives groups of health professions students from other foreign universities, including the University of Saskatchewan in Canada and Lund University in Sweden.

\section{Participant Recruitment}

My data comprised the spoken perspectives of physician preceptors, health facility administrators, medical officers (known as registrars in Tanzania), clinical officers, nurses, local trainees, patients, family members and community liaison members at the three host sites in Uganda and Tanzania. Community liaison members were individuals who were not in a formal clinical role and who had greater interaction with visiting medical trainees in the community outside of the hospital or health centre. I spent six weeks in Mwanza, Tanzania from September 2, 2017, until October 12, 2017, for the data collection phase of this study. I then travelled to 
Mbarara, Uganda where I stayed six weeks from October 13, 2017, until November 28, 2017, for data collection. I scheduled initial meetings at each host site with the local research collaborators to build rapport and identify strategies to recruit participants for the focus group discussions and semi-structured interviews.

At each host site, purposive sampling was used to identify host stakeholders from each of the stakeholder groups. Individuals who were likely to have experience interacting with visiting medical trainees were selected for individual interviews or focus group discussions. These participants were identified by the low-and middle-income country (LMIC) research collaborators at each research site. Identified host stakeholders were then contacted in a variety of ways determined to be most appropriate by the LMIC research collaborator: 1) The majority of participants were contacted via mobile phone; 2) A few participants were contacted via email; 3) Nurses in Tanzania were invited to participate in a semi-structured interview through distribution of posters on the hospital wards; 4) Patients in Tanzania and Uganda were invited to the study in-person, or by mobile phone for outpatients, by a local health care provider prior to contact by the researcher; 5) Snowball sampling was used to recruit medical trainees for the focus groups after one identified trainee was contacted via mobile phone.

Local healthcare staff identified patients and their family members who were appropriate to invite to participate in the study. The study information was then provided to the patient or family member by the local staff (i.e., consent form in either Swahili or Runyankole), and if they indicated interest in participating, those patients and family members were then invited to attend a focus group. Only patients who spoke English, Swahili, or Runyankole were recruited, who were not acutely ill, over 18 years old, and whom host staff felt would be comfortable partaking in a focus group discussion. 
At the time of the phone call or email, potential participants were made aware of the topic and purpose of the study, the expected time commitment, and emphasis was placed on the voluntary nature of this commitment. At the time of face-to-face contact, the interview moderator shared the specific details of the study, as well as the possible benefits or risks, participants' privacy, and consent.

\section{Data Collection}

\section{Interview and Focus Group Process}

To reduce the potential power differential and minimize courtesy bias, a local moderator conducted thirty of the thirty-two semi-structured interviews and all focus groups. (Green \& Thorogood, 2014) I did not participate in any, except two, of the interviews and I did not participate in any of the focus groups (see paragraph on 'language' for these two exceptions). I spent significant time prior to the interviews and focus groups with the moderators and transcriptionists to familiarize them with the study. The following table depicts the number of interviews and focus groups conducted at each site.

Table 2 Number of Interviews and Focus Groups Conducted at each Host Site

\begin{tabular}{|l|l|l|l|l|}
\hline & $\begin{array}{l}\text { Mwanza, } \\
\text { Tanzania }\end{array}$ & Mbarara, Uganda & Rugazi, Uganda & Total \\
\hline Semi-Structured & 16 & 11 & 5 & 32 \\
\hline Focus groups & 2 & & 1 & 6 \\
\hline
\end{tabular}




\section{Mwanza, Tanzania.}

Through email contacts via one of my committee members, I was able to identify an experienced interview moderator as well as two transcriptionists in Mwanza prior to my arrival. On my arrival, I met with the interview moderator and transcriptionists to explain the study, their roles within the study, and remuneration for their work. Upon determination of their suitability for the respective positions and agreement on the remuneration, I hired one interview moderator and two transcriptionists in Mwanza. With the assistance of the local research collaborator, we were allocated two secure offices within CUHAS, which is located on the third floor of the main hospital building. The remaining floors of the eleven-floor building contained inpatient wards. Therefore, it was very convenient for the majority of participants to participate in the interview and focus groups in this office as it was free from distractions from other people but did not require any travel time. However, for those participants who preferred to have the interview in their own offices, we complied with their wishes. The second office was used by the transcriptionists for transcribing. This was convenient because if there were any questions related to the audio recordings, the interview moderator and myself were located nearby.

\section{Mbarara and Rugazi Health Centre IV, Uganda}

On my arrival in Uganda, an interview moderator and transcriptionists were identified by the LMIC research collaborators as having the appropriate experience necessary. Similar to Tanzania, I interviewed the identified individuals and upon agreement of their responsibilities and remuneration, proceeded with the data collection. At MRRH, I did not have dedicated office space, and therefore the majority of interviews took place in the participant's office. However, for all of the trainee focus groups, I reserved a quiet space in the Simulation Centre within the 
MUST campus. Given the logistical issues of arranging lodging for the entire research team at Rugazi Health Centre IV for several days, transport was arranged, and we travelled daily to Rugazi Health Centre IV on three different days to conduct a total of five interviews and one focus group. At Rugazi Health Centre IV, the majority of interviews and the focus group discussion were conducted in the classroom on the hospital grounds that provided a quiet space without interruptions.

\section{Semi-structured interviews}

I used semi-structured interviews, to gather data from health facility administrators, physicians, nurses, clinical officers, medical officers and community liaison members at the three host sites in Uganda and Tanzania. The interviews ranged in duration from seventeen minutes to eighty minutes in length; the majority were approximately forty-five minutes in length.

\section{Focus Groups}

I used focus groups to gather data from local trainees (residents, interns, and medical students), and patients and family members in the two different host countries. My rationale for conducting focus groups, rather than semi-structured interviews, with these stakeholders, was that they might have felt uncomfortable in an interview setting and might have been more likely to contribute personal accounts through listening to each other's accounts. The focus groups in the host countries were divided into different groups to minimize power dynamics due to hierarchical constructs, and to encourage open discussion. Initially, I planned to conduct a focus group with medical officers, however, despite recruiting six participants for the focus group in Tanzania, only two participants came, so this became a two-person semi-structured interview. In 
Tanzania, the medical trainee focus group was comprised of a range of local trainees from medical student level to resident level. However, in Uganda, it was determined, in collaboration with the LMIC research collaborators, that due to potential power dynamics it was better to separate trainees into different focus groups according to their trainee level. Therefore, in Uganda three separate trainee focus groups were conducted with the following groups: 1) Residents, also known as Master of Medicine (MMED); 2) Interns; and 3) Medical students. A focus group was conducted with patients and a patient's family member in Tanzania, whereas in Uganda a focus group was conducted with only patients. Again, the reason for the variation in participants in the patient focus group was due to logistical issues. The focus groups lasted approximately one hour in length.

\section{Interview and Focus Group Format}

I used a semi-structured format establishing the participant background, exploring their experiences with visiting medical trainees, exploring their expectations of visiting medical trainees, probing whether there were any benefits or harms of visiting medical trainees coming to their institution and community, and exploring their reflections based on improvements for global health electives to ensure fairness, reciprocity, and mutual benefits (see Appendices C and D for interview guides). In Tanzania, due to budget constrictions, I was not able to hire a notetaker to take notes during the interviews; however, I did hire a note-taker for the three focus groups in Swahili. In Uganda, I employed the transcriptionists also to take notes during both the interviews and focus groups. Their observations were then incorporated into the transcriptions. The note-takers in both Tanzania and Uganda made notes in English regarding their observations in terms of body language, atmosphere in the room, and facial expressions which would not be captured by the audio recordings. Immediately after each interview or focus group, I debriefed 
with the interview moderator. At all sites, participants received light refreshments at the time of the interview or focus group as appreciation for their time commitment.

\section{Language}

In Tanzania, all but two, of the interviews and focus groups were conducted in Swahili by the interview moderator. I conducted two of the interviews myself with physicians who spoke fluent English. In order to ensure that appropriate probes were used to garner in-depth information, I conducted semi-structured interviews with these two Tanzanian physicians in English. The interview moderator sat in on these interviews so she could better understand the interview format and when to probe. However, following these interviews, I felt that I would not acquire more in-depth information if I conducted the interviews with participants who spoke fairly proficient English, and therefore decided to continue the process with the interview moderator conducting the interviews in Swahili. I did not participate in any other interviews or focus groups.

In Uganda, given that English is the official language and the majority of healthcare professionals speak proficient English, the interview moderator conducted all of the interviews in English, except for two interviews which were conducted in the local language Runyankole. One of the interviews in Runyankole was with the patient focus group as the majority did not speak English and the second one was with a community liaison member who was also more comfortable speaking Runyankole.

All of the interview and focus group guides were translated into Swahili and Runyankole. To ensure that participants would understand the questions, I reviewed the translations with the moderators to ask whether appropriate terminology was used by the translators. All of the 
interviews were audio recorded and then directly transcribed into either Swahili, Runyankole, or English. The transcripts in Swahili and Runyankole were then translated into English. Due to difficulty in finding an independent translator in Uganda within a limited time period, the interview moderator translated the two Runyankole transcripts into English. An independent translator in Tanzania translated the Swahili transcripts into English. The interview moderator or the local research collaborator read all of the translated transcripts and compared them to the original transcript or audio recording to ensure accuracy of the translation. I reviewed all of the English audio recordings to ensure accuracy of the transcriptions.

\section{Field Notes}

I kept field notes throughout the data collection and data analysis phases of the research process. Recording observations allowed me to make connections between my interview data and my observations. Noting observations was a challenge on several levels because I was not present during the semi-structured interviews and observations were, therefore, second-hand from either the moderator or the note-takers. Also, a note-taker was not present during the Tanzanian semi-structured interviews, and therefore it was difficult to obtain in-depth observations as it is impossible for the moderator to fully focus on this aspect of the interview. Important descriptive observations from the focus groups in Tanzania and all semi-structured interviews and focus groups in Uganda, including body language, tone, facial expressions, perceived moments of emotion (both positive and negative), and other essential elements were recorded and then connected to the data to provide richness to the stories that were told. Field notes provided valuable contextual data, increased rigour and trustworthiness, and emphasis on important points being made by the participants. (Phillippi \& Lauderdale, 2017) This assisted in 
the interpretation and analysis of the data in terms of understanding the perspectives of the participants.

\section{Data Analysis}

Once transcribed, I imported the interviews and focus groups into NVivo 11. Data was analyzed by myself and independently by my research committee to identify emerging themes. I used the stages of coding as described by Smith et al. (2003) as my analytic method. Braune and Clarke (2013) set out a step by step guide for the stages of coding in interpretive phenomenological analysis (IPA). The purpose of coding in IPA is to understand the participant's meaning and their experiences to thereby enable interpretation. It is therefore not intended as a method of data reduction, but it is part of the analysis. (Braun \& Clarke, 2013) I was active in interpreting the participants’ experiences but needed to bracket off my initial ideas and assumptions to focus on the participants' meanings.

I familiarized myself with the data by listening to each English audio recording at least once and reading the transcripts multiple times, taking note of items of potential interest in the data, in relation to the research questions. Complete coding occurred focusing first on one data item (i.e., transcript of one interview or one focus group) at a time. Coding involved a close (line-by-line) reading of the data, but I assigned codes to as small or large segments of data as relevant. The coding in IPA may be anything of potential interest in the data, in relation to the research question and can be descriptive (data-derived), conceptual (researcher-derived) or linguistic. After coding one entire data item, I moved on to developing emergent themes within that specific data item. Searching for connections was done across emergent themes within that data item, and superordinate themes were generated. A tabular representation of the analysis for 
the first data item was produced. These steps were then repeated for all the other data items.

Finally, themes and superordinate themes were identified across the entire dataset, and a final figurative and tabular representation of the analysis was produced. A flow diagram illustrates the steps taken in my data analysis based on the process outlined by Smith et al. (2003)

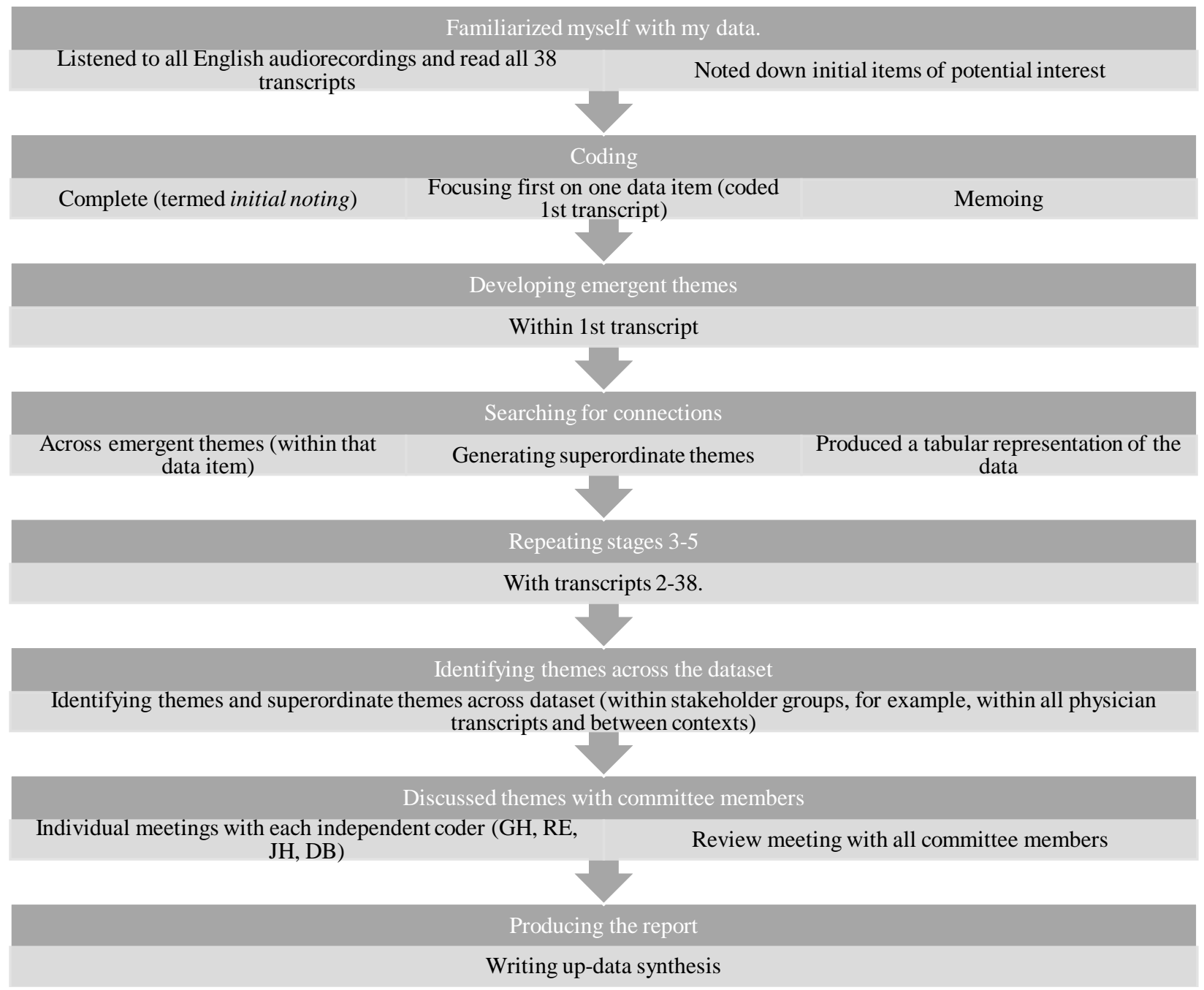

Figure 1. Flow Diagram of Data Analysis using IPA

Three other members of the research team (GH, DB, JH) equally divided the 38 transcripts amongst themselves and did independent line-by-line coding of 12 or 13 interviews and/or focus groups each. One other member of the research team (RE) coded all of the 
interviews. Both $\mathrm{GH}$ and $\mathrm{JH}$ have significant long-term experience in the field of global health research and education, especially in fostering strong partnerships between Canadian institutions and LMIC institutions. RE is an expert in medical education and qualitative methods. DB is both an expert in qualitative methods and has previous experience conducting research in LMIC. Emerging themes were discussed with the entire research team including the research collaborators in Uganda and Tanzania.

\section{Ethical Considerations}

I considered the target population for this study vulnerable in several ways: some of the participants were patients, all participants were living in a low-and middle-income country, and participants may have perceived that involvement or lack of involvement in the study may negatively affect their care or their position. Participation in this study may have caused some inconvenience to the participants, including the use of their time. To address these potential challenges, I used the core principles outlined in The Tri-Council Policy Statement (TCPS 2) to guide my study. (Canadian Institutes of Health Research, Natural Sciences and Engineering Research Council of Canada, \& Social Sciences and Humanities Research Council of Canada, 2010) This is a collaborative document that outlines the ethical considerations and obligations that must be adhered to when performing research involving human subjects. The three core principles are respect for persons, concern for welfare, and justice.

\section{Respect for Persons}

Respect for persons incorporates the responsibility to respect the autonomy of research participants. An essential mechanism for ensuring a participant's autonomy is to obtain their free, informed, and ongoing consent. Every effort was made to ensure participants understood 
that their participation would not affect any health care or services they might receive in the future and would not affect their position or job in any way. Participants were informed they could choose not to answer questions they did not wish to respond too and that they could leave at any time during the interview or focus group. Consent forms were translated and printed in Swahili or Runyankole for participants who were not fluent in English to ensure fully informed, voluntary participation. Signed voluntarily, consent forms were obtained from all participants.

\section{Concern for Welfare}

Concern for welfare means that researchers should aim to protect the welfare of participants and to provide participants with enough information to adequately assess risks and potential benefits associated with their participation in the research. Respect for privacy and confidentiality is also an element of concern for welfare. Participants were not subjected to any unnecessary harm or discomfort. Potential harms include physical, psychological, emotional, social, financial or other risks. The most probable element of harm that could have occurred from participation in this study is that participants in Uganda and Tanzania might have felt psychologically distressed, anxious, or scared due to the power differential between them and myself. Participants may have also felt distressed due to language and cultural barriers between them and myself. This was addressed by having a local moderator conduct the interviews and focus groups in the language of preference for the participants.

I also had a responsibility to acknowledge potential benefits for subjects themselves or the community as a whole. Although participants were informed that there may or may not be a direct benefit to them, their perspectives would help us to better understand how global health electives could be improved to make them more beneficial to all stakeholders involved.

I took measures to protect the privacy of participants and the confidentiality of the 
information they shared. Maintaining a safe and private environment during the interview was a constant priority. Interviews and focus groups took place in a quiet and private location. Measures were taken to protect the participants' identities throughout the data collection and analysis phases. Identifying information was not included in the transcripts and each participant was assigned a code. Transcriptionists at each site were instructed to omit all identifying information from the transcripts, before giving them to me for analysis. For example, instead of recording the participant's name, they recorded "participant A_01” in its place. Confidentiality of the data was assured by storing the digitally recorded data on an encrypted, passwordprotected server. Anonymous electronic transcripts for each site were shared among the research team members for analysis purposes, through a secure email server or via direct transfer. Analysts, except the PI (Dr. Gwen Hollaar) who is required to keep the data for five years, will delete electronic files and destroy any paper records of the transcripts upon completion of the study. No identifying data was used in the analysis such as name or date of birth. Participants were only identified by their role in the final report and are not identified with a particular hospital. Only aggregate data is reported. No individual data is reported, except for some brief unidentified quotes that are used to illustrate specific findings. Participants will not be directly identified in any resulting reports or publications.

\section{Justice}

Justice refers to the fair and equitable conduct of research with a significant component being the recruitment process. Participation should be based on the inclusion criteria that are justified by the research question. In our study, I attempted to include all host stakeholders who had experiences with visiting medical trainees and did not exclude participants based on race, religion, ethnicity, or gender. An important threat to justice is the imbalance of power that may 
exist in the relationship between researcher and participant. Participants will usually not comprehend the research to the same extent as does the researcher. Again, this was addressed through reviewing the interview and focus group guides with the local moderators and research collaborators to ensure the questions were written in a way that would be understandable and not unduly distressing for the research participants. As already discussed, to minimize power imbalance, the interviews and focus groups were conducted by a local moderator.

\section{Ethics Approval}

Ethics approval was granted by the University of Calgary Conjoint Health Research Ethics Board (CHREB) (REB 17-0483), the ethics review boards at MUST (No. 15/07-17), CUHAS (CREC/222/2017) and the Uganda National Council of Science and Technology (SS229ES).

\section{Limitations}

I anticipated several potential limitations and this informed the study design to attempt to mitigate these limitations. These limitations included budgetary and time restrictions, linguistic and cultural limitations.

Budgetary and time restrictions had an impact on the length of time that I had at the research sites to establish relationships with local partners and engage in the data collection process. Also, budgetary restrictions limited the number of local research assistants I was able to hire minimizing the depth of information gathered (i.e., observational data). Thus, I selected research sites where there was a pre-established support network to mitigate this limitation. The 
associations through long-standing institutional collaborations between the $\mathrm{U}$ of $\mathrm{C}$ and the host sites helped to establish support before my arrival in Mwanza, Tanzania and Mbarara, Uganda. I was able to meet in person with a research collaborator from both Mwanza and Mbarara in Calgary prior to my travel to the research sites.

I considered other methodologies associated with the qualitative approach, such as ethnography and grounded theory. An ethnographic approach would have allowed specific insights into the social structure and local culture around global health electives. Ethnography also involves prolonged engagement with participants, through living and working with the community, so that the researcher comes to see the world through their eyes and understand in detail how beliefs are embedded in local cultures. (Green \& Thorogood, 2014) Grounded theory as described by Glaser and Strauss (Glaser \& Strauss, 1967) seeks to develop an explanatory theory of fundamental social processes, studied in the environments in which they take place. Grounded theory relies on theoretical sampling which involves recruiting participants with varying experiences of the phenomenon until theoretical saturation is reached. The limited amount of literature available in regards to host stakeholder perspectives on global health electives would make grounded theory a desired methodology to study this phenomenon and develop an explanatory theory. However, due to the budgetary and time constraints described, these approaches were not feasible for my study.

Linguistic limitations were mitigated by the use of an appropriately trained moderator. Courtesy bias was anticipated given that participants may not want to jeopardize established partnerships. I attempted to mitigate courtesy bias by not being present during the semistructured interviews and focus groups to allow participants to feel more free in discussing their experiences and potential concerns. 


\section{Rigour}

Rigour within research involves demonstrating the validity of the findings. (Lincoln \& Guba, 1986) The establishment of rigour in qualitative research is guided by the principles of trustworthiness, including transferability, credibility, dependability, and confirmability. (Lincoln \& Guba, 1985) It is the responsibility of the researcher to demonstrate how the study addressed the issues of rigour. (Koch, 1994) This section will address each of the four principles of trustworthiness and discuss how each aspect was addressed throughout the process of the study.

\section{Transferability}

Assurance of external validity or generalizability in quantitative studies is similar to transferability in qualitative studies. (Shenton, 2004) The extent to which findings from this study are transferable and used to inform understanding in similar contexts is an important consideration. The data for this study was collected in three particular settings and populations that may not be applicable to other contexts. Sufficient details have been provided for the reader to appraise similarities in context and determine what would be meaningful and applicable in their own experiences. The findings likely share many parallels with other LMIC host stakeholder experiences with visiting medical trainees. Also, many other high-income country institutions send their medical trainees on global health electives, and the findings of this study may apply to their programs as well.

The participant demographics, as well as cultural, environmental, and logistical contexts, have been reported by the researcher to allow the reader appropriate insight to assess for this aspect of trustworthiness. Koch (1996) claimed that the researcher, through disclosure of decisions, events, and influences might establish trustworthiness of a study by recording these in 
a journal and reporting it to the reader. These items were recorded by the researcher as an addition to the bracketing process. An audit trail was also used to establish transparency of the data. (Green \& Thorogood, 2014) The audit trail was established by the researcher through notes, photos of environmental contexts, and documentation that will enable others to follow the process from raw data to isolated statements, formulated meanings, established themes, and the overall essence of global health electives as experienced by the participants in Tanzania and Uganda.

\section{Credibility}

Credibility indicates the legitimacy in the truth of the data and the interpretations derived. (Green \& Thorogood, 2014) Whereas measures to ensure internal validity are implemented in quantitative studies, measures to ensure credibility are similarly used in qualitative studies. The assurance of authenticity entails assessing the quality of the data and methods used in data collection and analysis. (Sargeant, 2012) A variety of techniques have been suggested for the demonstration of credibility, of which I used verbatim participant accounts, triangulation, and independent analysis of data by my thesis committee.

\section{Verbatim participant accounts.}

One way to ensure credibility is to determine that there is congruence between study findings and existing literature. (Shenton, 2004) Participant verbatim accounts are provided in Chapter Four and support the discussion of study findings in Chapter Five. Study findings are consistent with existing literature on LMIC host stakeholder perspectives regarding global health electives.

Participant interviews were recorded and transcribed verbatim. The limitation in this 
study is that the interviews which were in Swahili and Runyankole were translated into English and these translations were used for the analysis, which may result in some loss of meaning and therefore inaccurate interpretations. However, these limitations were mitigated by the procedures described earlier. Transcripts capturing participant responses precisely as stated provide an accurate account of their responses which were referred to time and again throughout the coding and data analysis processes. Ongoing reference to and incorporation of participant quotes in reporting of study results and discussion provided a means to ground and develop the theoretical approach, and ensured that the researcher remained close to the data. Further, providing the raw data and the researchers' interpretation of the raw data allows the reader to determine the credibility of the interpretation.

\section{Triangulation.}

Triangulation is described as a strategy, or an approach used, to control bias and improve the reliability of the findings of a qualitative study. (Green \& Thorogood, 2014) Triangulation often involves using several kinds of data to verify and strengthen study results. In this study, we used triangulation in two distinct ways: data triangulation and investigator triangulation. (Green \& Thorogood, 2014)

\section{Data Triangulation.}

Triangulation using the data occurred in different ways. First, I used data from multiple different sources or stakeholders to provide a variety of perspectives about the phenomena of global health electives. My aim was not to produce a consistent version of the phenomena of study, as that phenomenon is inevitably socially constituted, but to challenge the biases that come from only one perspective. (Green \& Thorogood, 2014) Triangulation of data, therefore, helps the researcher not to pin down a positivistic reality, but it is instead an approach to bring 
the essence of the phenomena more sharply into focus and unpack the experiences of LMIC host stakeholders in regards to visiting medical trainees.

The second source of data that allowed for triangulation was peer debriefing. At the end of each interview, debriefing took place with the interview moderator and/or the note-taker which was recorded in field notes. Peer debriefing is also a method to prevent bias and allows the researcher to consider alternate points of view. I also had many informal discussions with my research assistants, surgery residents, medical students, local research collaborators, taxi drivers, and other NGO staff in Tanzania and Uganda. Conversations included discussion around issues and topics that arose during the interview process to broaden my insight and perspective on these topics. This helped to minimize personal or cultural bias and was fully documented in the field notes.

\section{Investigator Triangulation.}

I had regular discussions with the Principal Investigator/supervisor (GH) throughout the study. Matters related to study design, preparation for interviews, interview process, study findings, and emerging concepts in data analysis were discussed. All of the committee members were independently involved in the analysis of interview transcripts. This study's Principle Investigator (GH), the co-supervisor (JH) and committee advisor (DB) each did line-by-line coding and development of themes for thirteen of the thirty-eight interviews and focus groups. The qualitative expert/committee advisor (RE) reviewed and commented on all thirty-eight transcripts. The comprehensive data with extensive corresponding initial and focused codes, as well as the proposed theoretical approach were assessed and discussed by the researcher and the entire committee. Study findings including the proposed model of the theoretical approach were presented to committee members for review and comment. 


\section{Dependability}

Dependability is akin to reliability or the 'repeatability' of interpretation in quantitative

research. In qualitative research, this is often interpreted as the likelihood that a similar piece of research would elicit comparable but not contradictory kinds of themes. (Green \& Thorogood, 2014) Therefore, auditability should be a criterion of rigour. To address this, the researcher should report the study processes in detail, thereby enabling a future researcher to repeat the work, although not necessarily to obtain the same results. (Shenton, 2004) Field notes and memos included documentation of the procedures taken through the study process, including recruitment, data collection, transcription, analysis, and discussion of findings. The reflective diary discussed as part of the pursuit of bracketing also included relevant entries about personal attitudes and beliefs that would allow an auditor to follow the decision trail.

\section{Confirmability}

Confirmability of study findings is achieved when auditability, credibility, and transferability are established. Confirmability is suggested as a criterion of neutrality in qualitative research as opposed to objectivity in quantitative research. (Guba \& Lincoln, 1981) Confirmability, therefore, refers to the study findings themselves, not to the objective or subjective stance of the researcher. The confirmability of this study was enhanced by the written evidence, indicating the activities throughout the research process which allowed for illustration of the thought processes that led to the study findings. The exercise of bracketing, as recorded in the reflective diary entries, as well as documented procedures and accounts of the data collection and analysis process also contribute to the confirmability of the data. 


\section{Bracketing and Reflexivity}

Reflexivity is an important principle of qualitative research in that researchers should subject their own research practice to the same critical analysis that they use when studying their research topic. (Green \& Thorogood, 2014) Although it is impossible for the researcher to stand outside their own values and subjectivities, reflexivity is one of the ways qualitative researchers have of taking subjectivity seriously and still be able to produce a useful account of the world. Reflexive bracketing is a method I used to set aside my previous knowledge and assumptions. One definition of bracketing as offered by Starks and Brown Trinidad (2007) explains that the researcher, “must be honest and vigilant about her own perspective, pre-existing thoughts and beliefs, and developing hypotheses...engage in the self-reflective process of “'bracketing”', whereby they recognize and set aside (but do not abandon) their a priori knowledge and assumptions, with the analytic goal of attending to the participants' accounts with an open mind.”

I acknowledged that my previous experiences contributed to my perspectives about the phenomenon of global health electives. As a medical graduate at the University of Calgary, I participated in two global health electives within African countries in a resource-constrained environment. I also participated in an educational role in developing and delivering a seminar for family physicians in Lao PDR. As a general surgery resident, currently completing a Masters’ of Science in Population and Public Health, my career goals include being involved in global health. I kept a reflective journal and memos of assumptions, judgments, beliefs, biases, experiences, perceptions, and presuppositions throughout the data collection and analysis phases. Continuing dialogue with the local research collaborators, moderators, transcriptionists and other individuals in Mwanza, Mbarara, and Rugazi assisted in putting my personal and Western beliefs 
aside, accepting the participants' stories as they were told, and placing these experiences and meanings within the context of the region, culture, and environment in which the participants live.

\section{Conclusion}

The goal of this study was to better understand participants' interpretations of the phenomenon of global health electives and to provide a comprehensive summary of these interpretations that would be most useful to others. To accomplish this, I used a hybrid of interpretive phenomenology and case study research methodology. A step-by-step description of the study process has been delineated. I discussed the essential elements of the research methods, while also addressing important points related to ethical considerations, limitations, and rigour. The process described allowed me to collect participant data and develop the analysis which will be presented in the following chapter. 


\section{CHAPTER 4: RESULTS}

\section{Introduction}

In this chapter, I will begin by outlining the demographic characteristics of the host stakeholders who participated in this study and then present the themes identified throughout the participants’ narratives. I identified three superordinate themes from the transcripts: 1) Global health electives (GHEs) are a common, yet indistinct phenomenon; 2) Challenges and burdens of GHEs; and 3) recommendations for improvement. GHEs as a common, yet indistinct phenomenon included themes related to the description of host stakeholder experiences with visiting medical trainees (VMTs): frequency of encounters with VMTs, VMTs are indistinguishable from other visitors, participants' understanding of why VMTs come to do a GHE, reasons as to why host stakeholders accept VMTs, what VMTs do at the host site, and the quality of interactions between VMTs and host stakeholders. The second superordinate theme focuses on challenges and burdens of GHEs including poor behaviours and attitudes of VMTs, the challenge of supervision of VMTs, VMTs strain physical and human resources, language barrier, GHEs are a one-sided relationship, and 'the muzungu effect' which relates to patient views' that VMTs are more knowledgeable than local health care staff. The third superordinate theme incorporated emergent themes related to the need for bidirectional exchanges and improvement of the process of GHEs both before VMTs arrive, during the GHE, and after VMTs leave the host site. Although these themes are identified across all host stakeholder narratives, there are important nuances within each theme and sub-theme which I will highlight.

\section{Demographics}

A total of thirty-two semi-structured interviews and six focus groups were conducted. 
Fifteen semi-structured interviews, one two-person semi-structured interview and two focus groups were conducted in Mwanza, Tanzania. Eleven semi-structured interviews and three focus groups were conducted in Mbarara, Uganda while five semi-structured interviews and one focus group took place in Rugazi, Uganda. The following tables illustrate the characteristics of stakeholders included at each site. All, but one, of the health facility administrators interviewed were physicians.

Table 3 Demographics of Host Stakeholder Participants in Semi-Structured Interviews

\begin{tabular}{|l|r|r|r|r|r|r|r|}
\hline Role & \multicolumn{2}{|c|}{ Mwanza, Tanzania } & \multicolumn{2}{c|}{ Mbarara, Uganda } & \multicolumn{2}{c|}{ Rugazi, Uganda } & Total \\
\hline & Female (n) & Male (n) & Female (n) & Male (n) & Female (n) & Male (n) & \\
\hline Health Facility Administrators & 0 & 2 & 1 & 2 & 1 & 0 & 6 \\
\hline Physicians & 2 & 2 & 0 & 4 & 0 & 0 & 8 \\
\hline Nurses & 4 & 2 & 3 & 0 & 1 & 0 & 10 \\
\hline Community Liaison Members & 1 & 1 & 0 & 1 & 0 & 1 & 4 \\
\hline Registrars & 1 & 2 & 0 & 0 & 0 & 0 & 3 \\
\hline Clinical Officers & 0 & 0 & 0 & 0 & 0 & 2 & 2 \\
\hline Total & 8 & 9 & 4 & 7 & 2 & 3 & 33 \\
\hline
\end{tabular}

Table 4 Demographics of Host Stakeholder Participants in Focus Groups

\begin{tabular}{|l|l|r|r|r|r|r|r|}
\hline Role & \multicolumn{2}{|c|}{ Mwanza, Tanzania } & \multicolumn{2}{c|}{ Mbarara, Uganda } & \multicolumn{2}{c|}{ Rugazi, Uganda } & Total \\
\hline & $\begin{array}{l}\text { Female } \\
\text { (n) }\end{array}$ & Male (n) & $\begin{array}{l}\text { Female } \\
\text { (n) }\end{array}$ & Male (n) & $\begin{array}{l}\text { Female } \\
\text { (n) }\end{array}$ & Male (n) & \\
\hline $\begin{array}{l}\text { Medical } \\
\text { students }\end{array}$ & 0 & 2 & 0 & 4 & 0 & 0 & 6 \\
\hline Interns & 1 & 0 & 0 & 4 & 0 & 0 & 5 \\
\hline Residents & 2 & 0 & 1 & 5 & 0 & 0 & 8 \\
\hline Patients & 0 & 2 & 0 & 0 & 6 & 0 & 8 \\
\hline Family Member & 1 & 0 & 0 & 0 & 0 & 0 & 1 \\
\hline Total & 4 & 4 & 1 & 13 & 6 & 0 & 28 \\
\hline
\end{tabular}




\section{Host Stakeholder Experiences with VMTs: GHEs are a common, yet indistinct phenomenon}

A superordinate theme that encapsulated the multitude of descriptive themes emerging from the semi-structured interviews and focus groups, was that GHEs were wellestablished at the three sites in this study. As one trainee perceived it: "people are used to it, it is business as usual, these people come, they work and go back to their home land." Themes identified that describe the commonly occurring phenomenon of GHEs from the host stakeholder perspective related to frequency of encounters with VMTs, VMTs are indistinguishable from other visitors, perceptions of why VMTs come for a GHE, why host stakeholders accept VMTs to come including perceived benefits to the host stakeholders, and characterization of daily interactions of different host stakeholders with VMTs. Varying, and sometimes contradictory, descriptions of experiences with VMTs by participants suggested that although GHEs are a commonly experienced and accepted phenomenon for host stakeholders, understanding of this phenomenon remained vague.

\section{'I have encountered many'.}

Participants described their frequency of encounters with VMTs. Some participants had limited personal interaction with visiting medical trainees while others had more contact. All participants were aware of visiting medical trainees coming to their host institution and community. Most participants reported encountering VMTs often without stating how often. A physician described his encounters with VMTs as a daily occurrence: "I encounter many of them, residents who come from different countries." Similarly, a community liaison member stated: "we receive so many different students from different universities [...] not really every month but at least once a year.” Both Ugandan and Tanzanian patients described repeated 
encounters with VMTs either during their hospitalization or as an outpatient. "I have encountered them on Saturday, on Sunday I didn't see them, I saw them on Monday and Tuesday, I have seen many," reported a hospitalized patient. Nurses, both in Tanzania and Uganda, also frequently encountered VMTs on the ward: "Very often, we work with them because at least in most of the days those students are here."

\section{VMTs come from a multitude of countries.}

Not only do participants encounter VMTs frequently, but they also reported that VMTs come from a multitude of countries. An administrator described this:

"I have encountered many people mostly from North America, that is United States and Canada...but also from Europe and I think a few who come from Australian University...”

Another administrator also reported that they receive VMTs from all over the world including trainees from within Africa:

"Umm quite a lot [...] to be sincere, so as an [senior administrator position] gotten visiting students from all over the world coming to the faculty of medicine [...] yeah, so that makes it like a global thing."

The majority of participants described frequent encounters with visiting medical trainees from a multitude of countries illustrating that visiting medical trainees coming to their health care facility and community is a common phenomenon that has been occurring for many years and continues to take place. Furthermore, given that VMTs come from all over the world, this is a global phenomenon and should therefore be of global interest.

\section{VMTs are indistinguishable from other visitors.}

Although the frequency of encounters with all host stakeholders establishes the 
importance of seeking all these host stakeholder perspectives, it was challenging to assess host stakeholder perspectives on only visiting medical trainees as defined in this study. This is because host stakeholders are inundated with 'foreigners' or 'whites' from numerous countries in numerous different roles. Despite explanation by the interview moderator at the beginning of each interview or focus group discussion who visiting medical trainees referred to, there seemed to be a lack of distinctiveness as to who these 'foreigners' are, as many participants appeared to consolidate all visitors into the same category as 'whites' or 'foreigners' when sharing their perspectives. Also, all of the institutions receive visiting medical trainees from many different high-income country (HIC) universities, and obtaining perspectives related to only University of Calgary VMTs proved impossible. The majority of health facility administrators, physicians, nurses and local trainees ascribed a greater degree of distinctiveness to VMTs than patients did and were aware of the different roles and education levels of 'foreigners'. Nurses referred to VMTs as students mostly without distinguishing between training levels. Local trainees in Uganda and Tanzania were even more aware of who VMTs are and that they differ based on their education level as one trainee described,

"But I think that working with them I think that it...depends first of all on [the] group in which they are, there are those who are undergraduates, there are MMED.”

Although local trainees may have the most awareness of the different education levels of VMTs, they still were not aware of precisely who is visiting since VMTs are often not introduced, as one intern explained:

“for us we just find them there, we don't know where they are from...we don't know at what level they are, some come as medical students... some as nurses, some come as residents, so we don't know them, some even come as specialists but to get experience from here, so we don't know their levels, who they are, what they have come to do..." 
Although most participants were aware VMTs come as learners and some were aware they have different levels of training, participants viewed VMTs as one learner among many. Another physician expanded on the issue of lack of distinctiveness in explaining that there are visiting students not only from high-income countries but also from other African countries, so "the picture is broad." In the above quotes, the participants mentioned that they are aware that VMTs are learners or students, but despite stating this awareness, often they would lapse into saying 'these whites' or 'foreigners' throughout the interview.

Lack of distinctiveness of roles amongst foreign visitors was most dramatically illustrated in the patient focus groups. In the patient focus groups, throughout the discussion they referred to VMTs as 'muzungu’ or 'wazungu' (plural), Swahili terms for a person of foreign descent. One Ugandan patient explained, “...those foreign students, you see for us we call them whites (abazungu) [she laughs].” Only one of the Ugandan patients was very clear in her understanding that these are students who are coming. Although trying to seek perspectives on only VMTs who complete clinical rotations at the host sites, I obtained perspectives on experiences with pre-medical students, research students from undergraduate to Ph.D., specialist medical doctors, and nursing trainees. Although in my findings I attempted to include quotes that related to VMTs, they may refer to other visitors. Participant perspectives were a melting pot of experiences with different 'foreigners' or 'whites'. There appeared to be multiple reasons for this lack of distinctiveness between VMTs and other visitors including that host institutions receive many visitors from foreign countries and host stakeholders are not always aware of their role or they are informed incorrectly about what their role is. Even those who were aware of the role of VMTs still described experiences with other foreign visitors, and thus it was evident that participant perspectives are an amalgamation of all these experiences. 


\section{Perspectives on why VMTs come to LMIC host sites.}

Host stakeholders provided several reasons as to why they believe VMTs come for a GHE, with some of those reasons differing from HIC perspectives on why sending institutions offer GHEs for VMTs. Participants perceived that VMTs come for three main reasons: 1) to learn about a) tropical diseases and b) challenges in a resource-limited environment; 2) the opportunity to touch and interact with patients; and 3) to become 'international doctors'.

\section{VMTs come to learn.}

Most participants perceived that VMTs come to learn. More specifically, VMTs come to their host institution to learn about tropical diseases and to experience working in a resource-constrained environment. Many participants recognized that their setting is an unfamiliar environment for VMTs. A nurse explained, "I think these people come here for the purpose of learning and they are very curious.” Most of the local trainees in Uganda and Tanzania concurred that although VMTs have good theoretical knowledge, they do not have much practical experience especially with tropical diseases:

"but what I've realized is that...some of the conditions that we have here are quite new to these people who come from outside, from those developed countries [...] they're not familiar to most of these infectious diseases that we have in our countries, for instance, I will give an example of malaria, uh, that some of these trainees who come they've never seen a case of malaria [...] they have a lot of theory but in the real practice, they need a lot of information that we do have."

Administrators, physicians, nurses, clinical officers, local trainees, and community liaison members all agreed that there is significant benefit to the VMTs in coming to their institution as they learn about tropical diseases, challenges in a resource-constrained environment, and 
different cultures. However, participants in both patient focus groups did not focus on the benefit to VMTs who come to low-and middle-income countries (LMIC), but instead focused on the benefits to themselves as patients. Only one Ugandan patient as described earlier appeared to be aware that VMTs come to learn. The majority of host stakeholders were aware that the main purpose of GHEs is as a unique learning opportunity for VMTs.

\section{'they get an opportunity to touch a patient'.}

A common assumption by participants was that VMTs are not allowed to touch patients in their home countries; LMIC host sites provide VMTs a unique opportunity to "touch patients”. A physician stated this common perspective as:

"... they come in with a need of a different experience where they are free, most of them in their countries are not free to touch patients, so here they can actually touch patients."

Although none of the Tanzanian administrators or physicians interviewed shared this perspective, five out of the ten nurses interviewed both in Uganda and Tanzania expressed this perspective. Ugandan and Tanzanian trainees echoed this same perspective. After one Tanzanian resident described her personal experience on exchange in a HIC and her difficulty interacting with patients in the HIC, another Tanzanian trainee responded:

"I have never gone to the field [to a high-income country] so it is something that we hear that when you go to them [HIC], you aren't allowed to touch the patient, they [VMTs] come to you, they touch the patient, how do you feel... a a it is a very big segregation. It is a moral gap but some customs differ but...it disappoints someone and discourages someone because they segregate you...the freedom he has here when they come to learn to our patient[s] and the freedom you have to their patients when you go there to learn is a big problem."

While most participants did not describe the perspective that VMTs come to 'touch patients' as upsetting to themselves, Tanzanian trainee’ focus group participants found this difference in 
patient interaction to be an ethical concern, because they perceived they would not be allowed to touch HIC patients if they went on an exchange. Only patients did not describe this perspective that VMTs are not allowed to touch patients in their home country leading to VMTs coming to a LMIC host site where they are allowed to touch patients. Notably, the majority of participants did not refer to what extent VMTs 'touch patients' and therefore no conclusions can be made as to whether VMTs are interacting with patients beyond their skill levels or without appropriate supervision. Participants perceived that VMTs come to their host site because of this unique opportunity to obtain more practical experience and 'touch patients' which they are not permitted to do in their home country.

\section{To become 'international doctors'.}

Another perception of why VMTs complete a clinical rotation at a LMIC host site was that they come to ‘learn global health’ or to train to become ‘international doctors’. Moreover, some participants were motivated to accept VMTs so they could teach VMTs to become 'international doctors’. One physician described his own experience during a GHE as a trainee:

"from that experience I really came to believe in the power of these sorts of global health rotations to both inform and to inspire...young doctors-in-training [VMTs]. I still believe that very much."

This physician believes a GHE can be inspirational for VMTs and potentially inspire them to do further work in global health. Another physician thought that European interns come because they are, "required to serve a period of training in developing countries before they can do their final

exam...” Other participants also emphasized that VMTs should be trained to be 'international doctors'. One administrator explained, "so it is easy for them to be international doctors that would help them to go anywhere in the world." Obtaining global health competencies also was 
perceived as requiring international travel. An administrator stated: "a student who gets an international exposure, their mindsets never remain the same. Yeah, there's a way they look at things differently, more holistically." Yet, it appears that host staff expect the local trainees to obtain a more holistic perspective through learning from VMTs:

“... a better learning opportunity for these local ones, because for example if we're talking about the health system in Uganda [...] then someone else tells us about their health system in Canada. I think that is a better way of learning than just confining ourselves to that one of Uganda."

Based on these participant perspectives, the definition of global health is likely understood very differently among these participants. Some participants appeared to view VMTs' learning about global health as a way to improve health inequities, others as a skill that would improve VMT career mobility, and some viewed it as a HIC academic requirement.

\section{Why host stakeholders accept VMTs.}

One of the reasons why participants accepted VMTs to come were to help VMTs achieve their own goals in coming to the LMIC host site as understood by the participants. Other reasons reported were the desire to portray themselves as welcoming, a moral obligation to teach all trainees, lack of choice to negotiate how many VMTs they supervised, and benefits for themselves as host stakeholders and the host institution.

\section{Welcoming culture.}

Participants' perspectives revealed a desire to be viewed as welcoming towards VMTs. A nurse explained,

"I make sure that I prepare positive environment, that is, to receive those students nicely and talk to them, in so doing you make their ability and understanding to be 
higher and they like your environment.”

Also, one of the residents described their culture as welcoming:

"But from our side, for the culture of our patients...I think as we have said, our culture is really welcoming, so uh, many of our patients are willing to accept to be worked on by the medical trainees."

This resident further described that patients are willing to provide consent to VMTs for procedures despite the language barrier. Given that a few participants commented on harms because of this willingness, it is interesting that this participant did not mention the potential for harm because of this welcoming culture by patients. Participants wished to portray a welcoming environment for VMTs, and this is likely part of the reason for accepting VMTs to come.

\section{Accepting VMTs is a moral obligation.}

Physicians and administrators were asked whether they felt it to be an obligation to host VMTs. Although I intended this question to mean whether host stakeholders felt they are forced to accept VMTs, the term 'obligation’ was interpreted by the participants in various ways. Many of the physicians and administrators felt it was a moral duty or obligation to accept VMTs in terms of the educational mission of their institution. For example, a clinical officer explained,

"I have told you every facility, every hospital, where you see a health center, where you see a hospital is a school. It is a school where people have to come and learn so it is an obligation. Yes, we should have students coming to... to the facility, yes."

An administrator viewed it as an obligation so that local trainees can learn from VMTs. On the other hand, a physician interpreted the question as I intended as he explained:

“No, we don't have to have them but I know there is... a global drive... they call it global health, that's what they have kind of called it and everyone in the Western world... ah mainly what I seem to see is that coming to Africa is part of their global health initiative a lot." 
This physician seemingly does not feel forced to accept VMTs but acknowledged that there is a drive or push from HIC institutions for LMIC institutions to provide global health opportunities for their trainees. Another administrator also does not feel forced to accept VMTs and clearly stated that they have the capability to say 'no' but that it is a mutual agreement and something done out of commitment. Also, participants who went on exchange to a HIC are motivated to provide a similar learning experience for VMTs. Although local trainees were not asked whether they felt it an obligation to accept VMTs, this theme did emerge as one trainee expressed:

"Yeah they come to seek for knowledge and if you don't support a person who is seeking for knowledge especially in health sectors/department it is like committing a sin because you might be the source for them to underperform and do things in wrong ways."

This local trainee, similar to other host physicians and administrators, perceived it to be either a personal moral obligation, institutional moral duty, or duty to society to provide VMTs with these global health experiences.

\section{Choice to Negotiate- 'no way of saying no'.}

A theme that emerged amongst physicians, administrators, and a few trainees was a lack in choice to negotiate the particulars of VMTs global health electives. As one administrator explained some VMTs are very insistent on coming at a particular time and this participant found it difficult to turn them down. This administrator further described:

“Yeah, so sometimes I think the lecturers wouldn't want to say yes but maybe someone is brought by a senior person, like you know we have this collaboration and this person is coming, so the lecturer has no way of saying no but in actual sense they would have said no, I think that's the biggest challenge I've got."

A physician also demonstrated his lack of choice to negotiate regarding how many trainees he 
can supervise:

"They just bring them [VMTs] and put them and say these ones need supervision, or they show up here [...] so basically they get off loaded onto you and they are there and you have to deal with them..."

This physician felt that once senior administration agreed to let VMTs come, he was forced to supervise VMTs regardless of whether he already had a significant load of teaching local trainees and patient care. He also further explained that VMTs are coming at random times throughout the year with some of the times being inconvenient, so he recommended a specific window of time in which VMTs could come. Similarly, a resident felt forced to supervise VMTs as she explained, "the next thing the chief resident would be called and she would be told 'there is this resident from this place, take her through."' The resident explained how this was very difficult given that she did not know the VMTs learning objectives or training level.

On the other hand, some administrators reported they are in charge of deciding how many and how long VMTs come for:

"so, of course, the biggest decision is to allow them to come and that is a huge one because you really sign, yeah, to allow them to come, and in allowing them to come how long they will stay that is key."

Although most host stakeholders do not feel forced to host VMTs, there is a theme of lack of choice to negotiate how many VMTs come and at what time they come. Lack of choice to negotiate appeared to be an issue between senior host administrators allowing VMTs to come and physicians and residents on the ward who feel forced to supervise them. Although participants perceived offering GHEs as a moral obligation and something that they wish to do, they advocated for more input into deciding how many VMTs come at one time, what time of the year they come, and how many they are involved in supervising. 


\section{Benefits to Host Stakeholders.}

All participants discussed benefits of GHEs. It was implicit that VMTs were valued because of a variety of benefits related to GHEs and thus, these benefits were rationale for accepting VMTs to come to their host site. The extent of perceived benefits varied greatly amongst participants. A few participants perceived great benefit from GHEs while a few felt there was little to no benefit. Benefits discussed related to mutual benefit, benefit to host individuals, benefits to the host institution, benefits due to reciprocity and collaboration, and benefits to global health.

\section{Mutual Benefit-Sharing knowledge.}

The theme of 'sharing knowledge and ideas' was mentioned throughout the majority of host stakeholder interviews and focus groups as a mutual benefit. Typically, participants expressed this benefit in general terms, without giving specific examples of knowledge shared. For example, a nurse stated, “...Yes, she [VMT] wanted to learn more from me but there were also things I wanted to ask...that we share together.” However, some stakeholders did give specific examples of knowledge exchange. In explaining what knowledge was shared some host stakeholders emphasized the knowledge they gained from VMTs rather than a bilateral exchange of knowledge. For example, one nurse reported:

"I also learn from them because when we are here we share and when we go on the ward they always say 'oh here we always do like this, we do like this' and they have a lot of what... information and knowledge, and ah they also help us and I also learn from them, some of the skills because they have their way of looking at things [...] definitely they help us..."

These perspectives illustrated that some participants found that VMTs taught them new knowledge that was applicable in their setting; however, not all participants agreed with this.

Local trainees in Uganda and Tanzania were most critical of how much knowledge they 
gained from VMTs. Although they did mention there was sharing of knowledge, the benefit to themselves was minimal. Trainees in the Tanzanian focus group felt that the differences in environment and available resources were too pronounced to be able to gain much useful knowledge from VMTs. A Ugandan resident echoed this perspective regarding the mismatch in sharing knowledge:

"it's a [VMT] resident giving other residents a lecture on things which our own residents actually perhaps even have more experience with the way [...] they are fitted for our setting and [...] you're a third-year resident and you're asking [...] yourself, you know, I'm a third year resident [laughter] I should be giving you a lecture on how, on how we do this repair here, not you giving me a lecture how we do the repair here. So, I think that mismatch is there sometimes ..."

In the patient focus groups, knowledge sharing was not discussed as a bilateral exchange but rather a unilateral transfer from VMT to patient. Patients expressed that VMTs provided advice, health education or explained imaging results to the patient. A few physicians and administrators mentioned that VMTs have better research skills and this was very helpful knowledge that VMTs shared with local trainees. In summary, sharing of knowledge, experiences, and ideas is an important component of GHEs with different perspectives expressed by all stakeholder groups. Some participants felt this knowledge exchange as mostly unilateral towards either the VMTs or themselves, whereas others felt it to be more of a bilateral exchange. Local trainees acknowledged but were more critical of how much benefit they derived from knowledge exchange with VMTs.

Benefits to Host individuals-VMTs as example to host stakeholders.

A significant theme that emerged is that VMTs act as a good example to host stakeholders in various dimensions. This typically was mentioned in terms of VMTs being a good example to local trainees, but also to nurses and other health care staff. One administrator 
however emphasized that both VMTs and local trainees could be examples to each other. VMTs were viewed as good examples in terms of bedside manner and work ethic. One nurse explained how VMTs’ behaviors toward a patient is something she would like to emulate:

"I mean the interpersonal relationship and the patients, how they take care of them, it is wonderful...I think that it is very good and also...they don't rush [...] I think we need to learn that one."

Some of the local trainees themselves felt that VMTs were an example to them as one resident said,

"they're hard working, you know, they come and they put in the effort and they put in the time [...] and they're very interested in, in patient care and that's I think one of the things that we sometimes need to learn from them, is that, you know, they're very passionate about it."

It is interesting that throughout almost all the interviews the main theme was how VMTs are a good example for local trainees, but not vice versa. Although patients did not specifically say that VMTs are a good example to host stakeholders, they viewed VMTs more positively as compared to local health care staff and local trainees which will be discussed later. Therefore, an important benefit as perceived by most participants is that VMTs provide a good example to local health care staff and local trainees in terms of certain positive attitudes.

Benefit to Host Individuals and Institutions-Gain of physical and financial resources.

A theme mentioned by participants at all host sites as a benefit was the gain of physical and financial resources. Patients also perceived great benefit in GHEs through physical resource gain as this patient described,

"and other things that we have benefited, we always hear that the whites sent services in the hospital or sometimes they send money sometimes to buy beds in the hospital, like there are some beds which we did not have in maternity there, they 
sent us money we bought beds [...] or you hear that they sent blankets, mattresses.” Very few participants felt that VMTs were a burden on local material resources but instead perceived that they gain physical resources which VMTs bring along with them or purchase and send after their GHE.

Benefit to Host Individuals and Institution- 'we see them actually as a big relief'.

Although VMTs are mostly understood to still be learners, there is a significant theme among administrators, physicians, nurses and trainees that they provide service and help relieve staff shortages. One administrator emphasized several times that VMTs are a relief especially in clerking patients: “No, they're not disruptive because as I've told you the staffing, we're understaffed... so we see them actually as a big relief." However, this administrator was quite clear that VMTs are learners and that their rural health facility also would like specialists from HIC to provide services such as surgeries. One nurse perceived that VMTs increased the efficiency of patient care by assisting with charting,

"also, another thing I have seen is that there is like ...there is a certain progress that when they see patients, they write in the files so it's like they have speeded up treatment somehow."

However, as one physician commented, visiting residents were perceived to be more useful in terms of service contribution compared to visiting medical students.

Not all participants felt that VMTs are helpful in providing service. One nurse stated that other people who come were more helpful than VMTs although it was unclear who is meant by 'other people': "Mm from other people who come here but I have never seen that [help] from students.” As further evidence, an administrator also said VMTs by themselves do little to improve the hospital: 
"Well it is not, when the students are there, there is some improvement but it is very difficult for you to really say the medical students by themselves have improved the hospital."

In their experiences with VMTs, many participants considered it a benefit that VMTs provide an element of service in terms of patient care.

Benefit to Host Institution-Elevates reputation of host institution.

Another benefit of GHEs discussed across many of the host stakeholder groups was that hosting and teaching VMTs elevates the reputation of the host institution. It makes the host institution more well-known and it promotes the host institution as a good teaching facility. As one administrator explained,

“...the University has a very good will for partnerships; they're very interested in people coming because it opens the University to the globe and uh, having a very good hospital, teaching hospital."

Physicians in both Tanzania and Uganda also shared this perspective. A slightly different perspective from a rural clinical officer was that VMTs not only elevate the reputation of their health facility and increase utilization of the facility by patients, but it also increases the confidence of patients in local health care workers. Local trainees and nurses also shared similar perspectives and several expressed that it places their institution "on the map”. Although in both patient focus groups it was not mentioned that allowing VMTs to come elevates the reputation of the institution, it was evident that patients find the care provided by the 'whites' as excellent therefore supporting the perspectives of the clinical officers.

Benefits due to reciprocity and building of collaborations.

Many benefits are perceived not to be due to VMTs or GHEs themselves, but due to GHEs 
already being part of a larger collaboration or leading to establishment of partnerships or collaborations, ongoing connections, or reciprocal exchange opportunities for host stakeholders. The perspective that an individual GHE is not beneficial on its own is illustrated clearly in this physician's statement:

"it can be beneficial to the host university but the benefit to the host university in my opinion really depends on the structure of the program and the commitment of the sending university to make the program mutually beneficial...”

An administrator also emphasized that GHEs are most beneficial as part of a collaboration: "or their [VMTs] presence can help us improve in some of the areas for example we have some relationship with [European university] and there are some big studies that have been done in collaboration with [European university]. There are ultrasound machines that have been brought in the department because of this relationship [...] We also have a good relationship with [American university], there are things like ECG machines that we have received as a result of this collaboration."

Some participants viewed exchange opportunities as a benefit resulting from GHEs. A nurse attributed the opportunity of travelling to a HIC because of the collaboration that also allows VMTs to come to her health facility. Connections with HIC specialist physicians were considered an important benefit resulting from GHEs as explained by a physician:

"therefore, through them [VMTs] we have managed to create a network with many people who are professors, so you communicate with a professor in neonatology or a professor of something, when we get a difficult case you share with them through email...it helps you in patient management."

Ongoing connections, even if only via email, were viewed by many participants as a benefit arising from GHEs.

It is evident that participants perceived that allowing GHEs at their institution leads to research collaborations, receiving of equipment, ongoing communication, and exchange opportunities with a HIC. The only stakeholder group which did not mention this perspective was 
patients. Although patients viewed VMTs as working together with local health care providers, they did not mention institutional benefits or reciprocal benefits because of GHEs. Across all stakeholder groups except patients, GHEs were not necessarily viewed as beneficial in and of themselves, but as part of collaborations, partnerships, ongoing connections, and the potential for exchange opportunities.

\section{What VMTs do at the host site- 'they come and go'.}

Participants varied in their descriptions of what VMTs do in their daily activities at the host site. Some participants perceived that VMTs immerse themselves in the daily activities whereas others felt that VMTs merely observed and did not interact much. One nurse only viewed VMTs as being present during the ward rounds, "They come and go.... in most cases during the ward rounds, when it is over they go." Other participants also described VMTs as simply observing and some were disappointed that VMTs did not actively participate.

On the other hand, there were also quite a few participants that perceived that VMTs actively participate in a variety of activities. A surgery registrar described that VMTs integrate well and complete the same activities as other health care staff:

"they are assessing patients during round visits, they are working together with indigenous students, the foreigners are doing the same just like other staff and native students do.”

Amongst all semi-structured interviews and focus groups there was significant variation on participant' perspectives in terms of what VMTs do during their GHE from mere observation to active participation. This difference in perspectives may also be due to misunderstanding or confusion about the level of training of the VMTs that they have interacted with. 


\section{Positive impressions of VMTs- 'really they have love'.}

Many participants freely shared their impressions of VMTs and frequently these were described as positive characteristics. VMTs were described as compassionate, loving, humble, patient, open-minded, careful, sensitive to local culture, actively participating, requesting help if needed, not stigmatizing, adaptable, and the list goes on. Typically, when VMTs were compared to local trainees or local health care staff by participants, they were framed in a positive light. A nurse repeated this comment about VMTs several times throughout her interview: "They are compassionate with patients...really they have love...and it's from their heart. You can see the way they act it's in a loving way/kind hearted." Something that was mentioned by several participants, including some local trainees and nurses, was that VMTs act quickly during emergency situations. Patients also described VMTs as caring, loving, and hard-working. One patient explained,

"there is a way he counseled me I felt I loved him, there is a way they handle you well, they don't want to talk to you rudely even though he was not hearing what we were talking but what the interpreter was telling him... .you would see that he really tries to love people."

Despite many of these positive impressions of VMTs, most of these participants also identify challenges as will be discussed later.

\section{Interactions between VMTs and host stakeholders.}

Perspectives on the quality of interactions between VMTs and host stakeholders varied greatly amongst participants. Many positive interactions were discussed, but also poor or negative interactions were described.

\section{Interactions with physicians.}

Most stakeholders interviewed perceived that interactions were positive between 
physicians and VMTs. One physician described how he helped VMTs to integrate well:

"My experience with them, well, many of them come initially when they are timid, they are not sure about what to find, but along the way, we help them get used to the environment and also get used to the people that they are going to work with. So, by the time they finish, they are almost like, you know, like part of us."

Many local trainees found that VMTs interact well with more senior staff: “.... they interact with everybody regardless of their title...” Also, interns found that VMTs' interaction with physicians was better than with other local trainees: "they tend to be engaged more with the specialists in the department." Although local trainees felt that VMTs interacted well with physicians, they found it disappointing that the interaction of VMTs was not as positive or open with themselves.

\section{Interactions with nurses.}

VMTs interaction with nurses was overall perceived as much more limited compared to those with local trainees or other physicians. There were several reasons mentioned as to why this might be: language barrier, nurse workload, and lack of introductions. A major difference in Tanzania compared to Uganda, is that Tanzanian nurses are less fluent in English and this limits VMT interactions with nurses in Tanzania. One nurse explained that although she speaks English, sometimes VMTs use difficult vocabulary which makes the interaction challenging. Also, some physicians found that VMTs interaction with nurses was limited. One physician believed this might be due to the heavy work load of nurses, which a nurse also described:

"none talks to them [VMTs], we never ask them if there is anything they need from us [...] I think it is because of our views and being busy with our commitments, people are busy with their activities that is all."

One physician explained interactions with nurses might be limited because VMTs did not 
introduce themselves to nurses. Several nurses also echoed this concern.

However, one nurse reported that VMTs work well together with local staff, including the nurses. Also, a health facility administrator explained that other health care staff speak well about their interactions with VMTs when the VMTs were not around:

"Me, I would say that the interaction with other staff is good [...] you see it is very easy to get information from people, the only way you can get information from people is by when these people [VMTs] are not there. So, you find that when they are not there they [other staff] really talk good about them. I have not got an incident where they talk ill or not well about them."

Although quite a few nurses described limited interactions with VMTs, there were a few nurses and other stakeholders who felt VMTs interacted well with nurses.

\section{Interactions with local trainees.}

Although many physicians and nurses perceived that the interactions of VMTs with local trainees were very positive, the local trainees themselves did not often describe this. Local trainees placed much emphasis on the importance of social interaction with VMTs and some found this to be limited as a medical student explained: "socializing with them is not that easy to say because first of all, different social settings, different everything so they don't really interact with us on a social basis." Similarly, several residents found the social interaction to be poor:

"But as my colleague was saying, we rarely encounter them in uh, extra academic activities, we tend to meet them while we're on the ward and on the ward we're specifically looking at the patients. So, I wish we could have social events where we can interact with them and we get to know each other very well. Much as their time here is limited but maybe if we can get that time to get to know each other very well, I think it could enhance what we get from them and what they can get from us." 
Not all local trainees were sure why the interaction is poor, but some reasons mentioned were that local trainees are busy, VMTs live at the expatriate compound, or VMTs are hesitant to socially interact with VMTs. Another local trainee explained that they do try to interact socially with VMTs: “at times you try to invite them for lunch and they're hesitant, they tell you how they came with their food well it's understandable, [group laughter] yeah, but the clinical areas they're very outgoing." However, several local trainees described positive interactions with VMTs as this medical student reported:

"those ones I interacted with them socially, academically, all sorts of interactions like, and socially they are actually fun people, down to earth, they try as much as possible to fit in our like...in our setting, which I think is very hard.”

Overall, local trainees were the most critical of VMTs' interactions with themselves. Although most local trainees found interactions on the ward to be positive or satisfactory, they found the social interactions to be quite poor.

\section{Interactions with patients.}

The interaction with patients was viewed as limited and this was mostly attributed to language barrier. However, many participants perceived that generally VMTs placed significant effort into interacting with patients despite the language barrier. As one nurse explained:

"they are used to be happy with the patients, they only fail how they can communicate...you feel sorry for them, you may find that maybe she wanted to help her but you find that she doesn't get that opportunity."

Another reason which stakeholders believed explained limited interaction of VMTs with patients was due to fear. A nurse explained her perspective:

"they fear they are going to catch what... a condition or a disease, so it depends, there are some are good, they interact freely, others get scared.” 
Interestingly, both Tanzanian and Ugandan patients viewed interactions with VMTs as positive and did not mention language barrier as a major challenge; instead they explained how foreigners provide good explanations even though they don't speak Swahili or Runyankole. VMTs were described to "attend well" and they "comfort" and "encourage”. The foreigners took care "with all their heart” despite language barrier. A patient described his perspective on an experience with a VMT: "They have done ultrasound to me, and they cleaned me, they did it very well, I'm thankful, and I thank them so much.” Although some participants viewed VMT interactions with patients to be poor, many participants, including patients themselves, perceived the interaction as positive despite the language barrier.

\section{Interactions with community.}

Participants viewed interactions with the community, outside of patient care, as mostly positive. A community liaison member described how VMTs tried to integrate well into the community:

"because [I] am remembering that way back, there is a group which also came and they said that they want to attend ceremonies, community ceremonies whether it is a burial or any that would be available, they go there and attend and they see how they behave, they are some who said that we want to attend church service they went and attended, with some we visited church leaders."

However, one administrator described a negative interaction with the community where VMTs were not careful in their interactions with the community:

"Some of them like interaction, they like much to interact with other people in the streets...something we don't recommend them so much... I remember one case that their dollars were stolen, they were in one hotel, their computer and dollars were stolen but we had warned them before, for example, we told them that we want to find them a place that is safe." 
Although most of the VMT interactions with the community, outside of clinical duties, were viewed positively by participants, this was one of the rare examples of a negative interaction.

All of these descriptions by participants suggest that although GHEs are a commonly experienced and accepted phenomenon for host stakeholders, a clear understanding of this phenomenon is lacking. Also, although participants overall tried to provide a positive summary of GHEs, the benefits and positive impressions appeared to be the silver lining as many challenges and burdens emerged.

\section{Challenges and Burdens of Global Health Electives}

The second superordinate theme focuses on challenges and burdens of GHEs including poor behaviours and attitudes of VMTs, unclear learning objectives, the challenge of supervision of VMTs, VMTs strain physical and human resources, VMTs take away learning opportunities from local trainees, language barrier, GHEs are a one-sided relationship, and 'the muzungu effect' which relates to patient views' that VMTs are more knowledgeable than local health care staff.

\section{No harms- 'I don't really see the harms'.}

Participants were reluctant to directly admit to harms of GHEs but described significant challenges and burdens of GHEs. One question asked of all participants was, "What do you think are harms in having visiting medical trainees come to learn in your hospital?”. The most common response to this question was an element of surprise and a declaration that there were no harms. One physician when asked this question expressed great surprise: "Harm! [Holds chin] [Moderator: yes] Of them coming? [Moderator: yes] Wow! I've not thought about it. Yeah.” 
Almost all administrators, physicians and clinical officers provided a similar response as this physician. Only one Ugandan physician and one Tanzanian physician described specific harms in response to this question. Another physician explained there was no harm as VMTs come for observation only. Most nurses, all trainees, and all community liaison members also declared there were no or minimal harms, when directly asked this question. Patients in both Uganda and Tanzania also stated there were no harms: "If these visiting medical trainees won't come here, it would be a big loss to us and not to them."

Interestingly, although most stakeholders declared there were no harms in VMTs coming to learn in their hospital, many of them also described challenges, harms, or burdens during data collection. However, when using the term 'harm' or 'disadvantage' they were swift to say there was no harm. Yet, inductive themes emerged from the interviews that there were significant challenges and harms of global health electives despite participants' reluctance to directly admit that GHEs do cause problems for host stakeholders and host institutions.

\section{Poor behaviours and attitudes of VMTs- 'these give us a bit of a problem'.}

Many participants described poor behaviours and attitudes of VMTs they encountered including arrogant and careless attitudes, cultural incompetency, inadequate emotional selfregulation and VMTs coming as medical tourists. Most participants explained they only encounter these behaviours and attitudes in a minority of VMTs, however others emphasized that this is a common and a significant concern.

\section{Arrogant and careless attitudes.}

Several participants, including administrators, physicians, and nurses reported that some VMTs display arrogant attitudes. One administrator described what he has heard from other host 
stakeholders: "I have heard a few, a few people uh, complain that some come with an attitude of 'knowing' but most are not...” A problem described by two nurses was that VMTs might have an arrogant attitude in acting like they know more than the nurses. One nurse described this, "But in some circumstances, it happens that when giving them some instructions there are some [visiting medical trainees] who understand and follow, but most of them have a certain attitude of feeling that 'this person can't help me', I don't know because I am an African and they are whites... These [VMTs] give us a bit of a problem..."

This nurse goes on to describe a very specific example of harm that occurred to a patient because some VMTs did not listen to her advice. This is a nurse who described that she enjoys teaching VMTs but emphasized the importance of supervision of VMTs because of her experience where some visitors did not listen to her advice. Another physician also explained how VMTs’ arrogant attitude leads to negative interactions:

“Certainly...certainly there have been times where students have...or residents have gotten upset on the wards, it doesn't happen very often, but then they'll start yelling at a nurse or you know yelling at another doctor and saying, 'Why...we would...in America we would do this, why don't you do this?' [...] again, doesn't happen very often but there definitely are those negative interactions occasionally."

Several participants described a similar arrogant attitude of VMTs where they did not heed host' warnings regarding personal safety issues.

A careless and insensitive attitude was also viewed as a poor characteristic of some VMTs as one nurse described:

"when they are done with their rounds they sit down on benches, sometimes they sit on benches where patients' relatives are supposed to sit, when relatives come they find them sitting on their benches all the time... when it is time to go they go."

Participants did not hesitate to describe negative or poor behaviours and attitudes of VMTs such as arrogant attitudes, careless and insensitive behaviours, and not paying attention to personal 
safety concerns. They viewed these behaviours and attitudes as leading to patient harm, harm to the VMTs, harm to the community, and an increased burden on host stakeholders in dealing with problems as a result of these attitudes.

\section{Cultural Incompetency.}

Many participants also described experiences related to behaviours that demonstrate the cultural incompetency of VMTs. A Tanzanian resident explained:

"There are some of them because of that advantage of being accepted by the patients she crosses the limits, when she comes the next time she doesn't [feel] any need of following any protocols [...] the other thing is for example you know to take some photos is prohibited unless you have a consent...someone does such things in your own ward without any...reason.”

This trainee explained that VMTs are crossing ethical boundaries by not requesting consent to take photos. However, this trainee found it difficult to address the harms of this with the VMTs as the patients were perceived as happy to interact with VMTs. Another nurse described how VMTs use of hand sanitizer was misunderstood and viewed negatively by patients: "some patients complained one time...yeah, [...] 'are we dirty are we what'... so it was not good.”

Another cultural incompetency relates to giving of financial aid or gift-giving. Although gift-giving or providing financial aid to specific patients by VMTs was mentioned by most stakeholders, only a few of them mentioned this as a challenge. One administrator explained how giving money to individual patients creates unrealistic expectations. In this administrator's experience, aid by individual VMTs was inconsistent and when it did occur, it created unrealistic expectations amongst the community that this administrator had to deal with. Another resident explained how VMTs were often unaware of the financial cost of ordering tests for patients. 
Additionally, some VMTs were viewed as culturally incompetent in relation to their social media use. A few participants shared experiences where the use of social media by VMTs was viewed as harmful. One administrator related his experience about a blog a VMT wrote:

"so the student documented not very nice things about anesthesia and surgery in Uganda that patients are not managed very well and, yes and so it was like a bad... portraying a bad image of the institution which is hosting you, yeah because much as we have problems we do not want you to put them in social...social media and things like that, but that was an isolated case, umm the student apologized and we cautioned her."

An earlier example given by a Tanzanian trainee related to VMTs taking photos without consent. Although it is not known how these photos were used, whether personal use or posted on social media, it was also viewed as an ethical concern by this Tanzanian trainee. Only these few participants mentioned the harmful effects of social media use by VMTs; however, many participants may not be aware of social media use by VMTs to document their GHE and therefore were unable to discuss the impact of social media use by VMTs.

\section{Inadequate Emotional Self-Regulation- 'they were getting cultural shocks'.}

Many participants, expect for patients, described emotional displays of culture shock or inadequate emotional self-regulation by VMTs. Emotional self-regulation is the process by which individuals influence the intensity, duration, valence, or manifestation of their emotional response to an internal or external event. (Sheppes, Scheibe, Suri, \& Gross, 2011) One physician in relating an example emphasized the importance of preparation of VMTs so that they can appropriately deal with the culture shock:

"the first groups of students we had were not very well prepared. They were getting cultural shocks and, [laughs] you know those first days were really very hard for them. I think some of them would even get depressed and so, we gave feedback and we said, you know what, let your trainees really know what is expected [...] yeah, 
I mean, there's one of them who could not believe it that a woman who has delivered can sleep on the floor and we told her, the space is limited. She burst out crying and then, she was honestly depressed for over a week."

Most participants did not describe the impact of the VMTs' emotional response on themselves and other host stakeholders. However, it is interesting to note that participants remembered these experiences very well and, in several descriptions, the VMTs were described as unable to overcome their initial culture shock. Another physician described how VMTs may be quick to display anger because of rigid expectations:

"you do get that one or two percent of students who are just simply not adaptable, so they will not...they have rigid expectations of how things will go and when things don't go that way they get very angry...um, so, and...and...you know, speak harshly to...to Tanzanian colleagues, um, that doesn't happen very often, because we have a more structured orientation I think...but it does...it still does happen.”

Based on the perspectives of participants, VMTs display culture shock both verbally and nonverbally. Although most participants did not directly discuss the impact of these experiences on themselves and other host stakeholders, the fact that they remember and describe these experiences illustrates that these were memorable experiences that are likely perceived as challenging to address.

\section{VMTs are viewed as medical tourists- 'I think it's a problem to have tourists'.}

VMTs were perceived as medical tourists based on host stakeholders' experiences. An administrator described that in his experience VMTs may have hidden travel objectives that are revealed after some time at the host site when VMTs request time to travel. Also, a physician illustrated the perspective that some VMTs view a GHE as a vacation:

"and I think there is a temptation for students and residents who are coming here to treat it as a vacation...it's a nice...you know, you're going to Africa...it's a vacation...you can go to the Tilapia in the afternoon...swim in the pool and drink 
a beer by the pool all afternoon but you came here to learn."

Another physician explained that he views VMTs as medical tourists when they want to see a little bit of everything in the hospital:

"I have had situations where students are in obstetrics and then they come and say 'oh, there is also pediatrics I also want to spend some two weeks in pediatrics' and there is no clear planning for that, there is no... they haven't even formulated why they want to be in pediatrics, so it tends to end up being a medical tour [...] and I think it's a problem to have tourists..."

One nurse reported that some health care workers also view VMTs as medical tourists: "I could say that sometimes some workers don't have any positive views towards them, they say these visiting medical trainees are just here like tourists, they are doing nothing.” However, no other nurses interviewed mentioned this perspective. A medical student also described how VMTs seem to come with a 'medical tourist' mentality and in his experience VMTs have prepared more for touring around (whether that is through different wards or to tourist sites, it is hard to know) rather than having prepared specific learning objectives. Another resident described his frustration with VMTs coming as medical tourists:

"I can guarantee you with $100 \%$ certainty that whoever student comes to this place, behind their objectives, is to go and visit the Queen Elizabeth National Park. Every one of them will [...] and the only time we shall talk about anything other than clinical or academic is maybe if you're asking them whether they went and what they saw and it gets boring..."

The data provides evidence for two reasons why VMTs are potentially viewed as medical tourists. One is that when VMTs come for a GHE, they may limit their clinical time to spend extra time sightseeing. The second reason is that VMTs were perceived as going from specialty to specialty for short periods of time leading to them being perceived as simply coming for personal curiosity and a wide array of opportunistic experiences instead of committing to a specified set of learning objectives. 


\section{Unclear learning objectives- 'they need to have specified objectives'.}

A prominent theme across almost all stakeholder interviews was that often VMTs come without clear learning objectives and this makes it very difficult to supervise them according to their training level, and to assess whether they are achieving their learning objectives. A Tanzanian trainee explained the problem of supervision due to unclear learning objectives:

"Then you are assigned/given to a person [VMT] whom you don't know where to place them because our studying systems [curriculum] are different from their systems...you don't know where you can place them, then there is no guidance on what you can do, at the same time, that person has no objective, what he needs."

A community liaison member echoed the problem of VMTs coming with unclear objectives and felt that this contributed to VMTs being perceived as a medical tourist:

"I feel that number one they are supposed to have the clear objectives... goals, objectives and goals clearly stated because I have seen some of them, [...] they come when they don't have clear objectives and to me I feel that almost their objective is touring so they should come up with clear objectives for the what... for the study.

Acknowledging that VMTs may have general learning objectives, a physician described that they do not have specific objectives. The nurses also commented that they were unsure whether VMTs do not communicate to them their learning objectives or if they simply come without specific learning objectives. As one nurse explained she is willing to assist VMTs, but if she does not know their objectives or what they would like to learn or become involved in, it is difficult. Based on these perspectives, it is evident that unclear or poorly communicated VMT learning objectives leads to challenges for host stakeholders in terms of supervision, assisting in their learning, evaluation, and it contributes to the perception that VMTs come as medical tourists. 


\section{Supervision of VMTs is challenging.}

Appropriate supervision of VMTs was viewed as very important by many

participants, yet this is a challenge as described by administrators, physicians, clinical officers, nurses, and local trainees. Some participants acknowledged that VMTs are required to be supervised when they come to learn to prevent harm. However, other participants explained that supervision of VMTs is challenging due to many students needing supervision and lack of staffing. A physician explained how supervising VMTs is viewed as a burden:

"every department is struggling with staffing even for our own students, so when you have to bring, sometimes you have ten on the schedule, sometimes we have groups from different sides they are all there, they need attention and you also are already busy with your own students, it becomes hard to attend to them."

Another challenge reported by a physician is difficulty in assigning VMTs to a specific supervisor as he does not know which physicians will be on the ward. Also, a rural clinical officer explained that if staff are away for a workshop, there may be insufficient staff to supervise and translate for VMTs, so this is challenging for the VMTs in that it limits what they

are able to do. Supervision was perceived as an important requirement to prevent harm when VMTs come, however this is a challenge faced by many host stakeholders. Challenges in supervision related to unclear learning objectives of VMTs, staff shortages, many local and foreign trainees to supervise, and a large burden of patients to care for.

\section{VMTs strain physical and human resources.}

Although many participants denied that VMTs are a burden, others acknowledged that VMTs coming has an element of added burden. Many participants felt that most VMTs bring consumables that they will be using such as gloves. However, a few participants mentioned that VMTs are a resource strain. One administrator explained that sending institutions should be 
responsible for VMT use of LMIC resources. A physician also explained that VMTs strain resources because they are not aware that supplies need to be re-used:

"they increase the strain on the resources we use, there they usually come from high income countries where you can afford to use resources and turn them a lot of supplies for one patient, I mean everything used for one patient, but here sometimes you need to use the same um...either sundries or something for many more patients so you find that the turnover of materials that we use to care for patients increases the time when they are here."

A few participants explained that VMTs may be a burden in terms of taking away limited accommodation. This was made even more challenging as VMTs might not always be happy with the accommodation arranged: "so you find that we are supposed to rent nearby and the only facilities which are available you find that those students are not comfortable with those facilities."

VMTs are not only a burden on physical resources but may become a burden on human resources when they are at the host site in large numbers. A physician related,

"The negative side is that they are demanding cause they can't speak the language, they don't know the culture, they don't know the environment they are in, so they put a strain on those who need to take them around or to deal with them every day and they end up kind of consuming people's time that would otherwise be used...I mean to deliver a service or to do work, so that one can be a downside."

VMTs not only take away health care staff's time from caring for patients but were also perceived as directly leading to increased burden on the patients as they are subjected to many learners. One administrator described the complaint he has heard from other health care workers regarding the large number of VMTs:

"I don't want to call it a harm but it could be a challenge if the numbers are not controlled, yes, so if you have so many students in...in a ward and then you add our medical students, so sometimes the department begins complaining 'now the number of students is more than the number of patients'."

Overall, participants perceived that VMTs are a greater strain on human resources compared to 
physical resources.

\section{VMTs take away learning opportunities from local trainees.}

Participants indicated that VMTs take away learning opportunities from local trainees and this was voiced mostly by the trainees themselves. All the Ugandan residents agreed that VMTs are prioritized by staff over local trainees: “Actually, to me what I have realized is that when the, these trainees are around, our local people tend to give much attention to those foreign trainees and ignore our local students.” Also, a medical student gives an example of medical students complaining that a visiting resident was limiting them from assisting in the operating room and completing the requirements in their logbooks. An administrator echoed this perspective:

“Umm I don't think, I don't want to call it taking it away, but then it brings in competition so if the numbers are big, yes, the... the time that our students may use to interact with the patients may be reduced [...] so it brings in competition that may reduce the opportunities yeah."

A Tanzanian nurse also related that learning opportunities decreased for local trainees when VMTs are on the ward. This nurse advocated for VMTs to be distributed to smaller hospitals or rural health centers to decrease the burden on their hospital. Although most participants were hesitant to say that VMTs take away learning opportunities from local trainees, it was a theme that recurred multiple times and therefore this challenge should be understood as a serious concern.

\section{Language Barrier.}

Language barrier was perceived by many stakeholders as a challenge that also strains human resources and can lead to patient harm; however, many participants accepted it as inevitable and described how they try to overcome it. Most nurses described the language barrier to be a 
significant challenge in that it limits their personal interactions with VMTs and that it might lead to harm or delay in patient treatment; whereas, physicians, administrators, and local trainees mentioned language barrier as less of a concern. Language barrier was perceived as more of a challenge in Tanzania compared to Uganda. As one Tanzanian nurse explained, "like I have said, language is everything, without language you can't do it, so really it brings misunderstandings, mistreatment, where a patient may not be treated accordingly." A Tanzanian physician acknowledged language barrier as a challenge, however it is a common challenge that he experiences even in his own country or when he travels: "Yeah I could say that the major challenge is language barrier, most of our patients...use Swahili and a few don't even know Swahili, so I also face some challenges when I face such a patient." Another pediatrician explained that parents are accepting of the language barrier and are amenable to local staff taking time to translate for VMTs. An administrator also explained that language barrier is a challenge, especially when interacting with patients, but that this challenge is common to both VMTs and local trainees:

“but it doesn't apply to only visiting students but even within our medical students there are those who don't speak Runyankole, so it is the same thing, so I don't think it is harmful but the patients may not be very comfortable if you don't use their language, because you have to use an interpreter in a process, yeah."

Many participants described how they try to overcome language barrier. Predominantly, this is through language interpretation, whether this is done by host staff themselves or by pairing VMTs with local trainees. One of the interns explained how language should not be a barrier to a VMT’s learning:

“they should be oriented that though they don't know the language, there are students who know the language [...] there are even actually caretakers who know the language, so really language should not be a barrier to them, uh, a barrier to their learning."

This intern demonstrated a willingness to help with language interpretation and does not describe 
it as a burden. Also, a Ugandan community liaison member felt that language should not be perceived as a barrier: "What about those doctors without borders? do they know each language? So, the issue of language it shouldn't be a problem." This participant perceived that local health care workers should know English fluently. A unique difference between Uganda and Tanzania, is that English is the official language in Uganda. However, some participants, mostly Tanzanian nurses, mentioned that VMTs should learn some Swahili to reduce the language barrier.

Interestingly, in the patient focus group in Tanzania, language was not brought up as a challenge or barrier. One Tanzanian patient described an interaction he witnessed where despite a language barrier it was not viewed as a challenge: "they were investigating that patient, then after that, they were explaining to her though they weren't speaking Swahili.” This patient perceived that despite a language barrier, the VMTs attempted to explain the patient's illness and the treatment plan (perhaps through an interpreter, although this is unclear from the patient's description). In the Ugandan patient focus group, language was mentioned as a barrier; however despite this, patients viewed all their interactions with VMTs as positive. One patient described her experience with VMTs when her child was sick and viewed the interaction as positive:

"I had my child s/he was sick [...] I saw they really cared for me, they even carried him/her and talked to me in Runyankole so that they can also learn it, we went there to maternity, they tried and I saw they were helping me on my child, ok for [...] me I feel happy."

From this patient's experience, it appeared the foreigners tried to communicate in some basic Runyankole. Despite a language barrier, the actions of the foreigners appeared to speak to the mother more than their words. In summary, although language barrier was viewed as a challenge, the majority of participants were accepting of this challenge and described ways they try to overcome it or how it should be overcome. 


\section{Psychological Harm.}

In addition to causing physical burden, VMTs were described as contributing to psychological harm of host stakeholders in various ways. One Ugandan intern explained that due to inferiority complex of local trainees, they are timid when VMTs are present and this limits their learning:

“ $m$ m, there's no physical harm really but there's a little bit of a psychological harm in such a way that uh, our medical students tend to be, to be timid. They tend to assume that they, they don't know and these foreign students know [...] so it will one way or the other affect the learning process if the medical students in Africa or in this place still continue feeling timid and withdrawing themselves away from these people."

Another way in which VMTs cause psychological harm is that their inadequate emotional selfregulation is challenging to local health care staff as this physician explained:

"And the other issue is that they are not used to certain situations so they can also be emotionally draining... I have had to deal with students who break down because they have not seen a child dying and when one dies they basically break down and they have to deal with their emotions yah and that can be straining emotionally...to everyone who is working with them they can really be straining."

Although earlier examples were provided of participant' experiences dealing with inadequate emotional self-regulation in VMTs, this is one of the few participants who described the personal impact of this behaviour. Also, a Tanzanian trainee provided an example of psychological harm as she explained how she felt bad when VMTs feel sorry for local trainees having to deal with the lack of resources in caring for patients. Interestingly, one of the Ugandan trainees perceived that the psychological harm is to the VMTs and not to the local trainees:

"I think some of the things they witness are a bit traumatizing [...] sometimes they find us working in those conditions that are depressing and I think, I think to them, this may hurt their mind [sighs, someone laughing softly] for us we have seen these 
things and all over it appears to be normal but for them sometimes it's very strange the things we do, and I think it kills the spirit in them. And they may, you know, they may not like uh, to, you know, to venture in some specialties, yeah."

This resident was concerned that some of the events VMTs experienced in the LMIC environment were traumatic and would prevent them from selecting certain specialties. As illustrated earlier, although many nurses described the emotional reactions of VMTs, such as shock and fear, to the LMIC environment they did not mention that this was emotionally draining for themselves. However, based on the perspectives of these host stakeholders who described psychological harm for themselves and other host stakeholders, this harm may be underestimated.

\section{One-sided relationship.}

Given the significant challenges and burdens described, GHEs are viewed as a one-sided relationship by many participants as GHEs mostly benefit VMTs with limited opportunities for host stakeholders. Participants provided explicit examples of how the relationship with HIC academic institutions is mostly one-sided, and they advocated that this should not be the case. For example, one physician described his experience related to a surgical camp that was not mutually beneficial in its initial proposal:

"We, we had an experience in 2009, where one of the faculties from one of the Universities I will not mention [laughs], from the west, came with about seven residents. And they came for a surgical camp. And all they wanted our faculty and residents to do was to clerk patients and not directly participate in the surgeries. So, learning out of that experience, we told them no. If you're to come, primarily our students have to learn. They must actively participate. And uh, so we had a little bit of a scuffle, and we told them if you don't want, stay there we stay here in our own world. Yeah. So, we had to reach an amicable position to agree on the way forward."

Trainees felt that the relationship is significantly biased towards benefitting VMTs. A resident 
explained how he viewed GHEs as a one-sided relationship: "but I think the most important of them is that this is really not an exchange program, it's a one-way traffic." A physician emphasized that GHEs that are not structured within a reciprocal program are not ethical: "and that's where I think the relationship has to be reciprocal...it's not ethical if it's not reciprocal...because even...even the best rotation, if you have students coming, there's gonna be some work that has to be done by the receiving institution...um, even for the best students with the...in the best program."

Although nurses did not directly state that the relationship is one-sided, many explained that there is little benefit to them. Another nurse felt that host stakeholders provided training benefit to the VMT, yet she does not see any long-term benefits for the host: "The only challenge is, they come here, we train them and then they go away, they take away the experience and they don't apply it so they are here for a short time." She further explained that she would like VMTs to return once they are fully-trained so that GHEs become mutually beneficial. Although patients had suggestions for how they would like to improve the relationship, they did not view the relationship as one-sided because their perspective is that VMT presence is beneficial to them.

Research by VMTs was also viewed to be skewed and provide more benefit to one side in various dimensions: ethics approval process, authorship, and research applicability. Ugandan residents explained that VMTs coming for research appeared to receive ethics board approval more quickly than local trainees:

"Maybe in the same line, if an international student came purposely for research, it would take her an average of two weeks to go through FREC, go through IRB and to go through the hospital administration to get clearance for all that. An indigenous student here would take months, and so people have been asking themselves what's the difference? Is it the money, is it the color, what's the difference? And I think it's not their problem; I think it's the problem of the hosts. It is a harm that is cropping up that people are beginning to question very many things. Why this favorability to these guys?" 
His perspective that VMTs are given these favours by the local ethics board, raised questions in his mind whether this discrimination is based on race or socioeconomic status. Several other participants mentioned that when research is done by visitors, authorship should be allocated fairly. A Tanzanian trainee mentioned that when a VMT comes to do research, it is not applicable to the local context and therefore the findings are not locally useful because they do not have the technology to implement the recommendations. Almost all participants, except for patients, viewed the relationship in regards to GHEs to be one-sided because VMTs benefit the most, there are unequal research relationships, and unequal exchange opportunities.

\section{'The Muzungu Effect'-an ethical concern.}

‘The Muzungu Effect’ refers to the patient' view that VMTs are more knowledgeable than local staff. Although host staff did not view this as a challenge, it needs to be recognized by sending institutions as having potential to lead to ethical issues. This theme recurred many times across all host stakeholder interviews and is called 'the muzungu effect' by one physician:

"the patients get excited especially if ah the muzungu effect... here 'eh the muzungu has touched me' and they get excited... ah that is quite interesting and then the families...some cannot distinguish between a muzungu who is qualified and a muzungu who is a visiting student that's a challenge, so the good they don't usually overstep their boundaries... so they don't cause harm but the families get excited quite often, yes.”

This physician described the challenge that patients are often not aware of the training level of the foreign visitor. However, his perspective was that 'the muzungu effect' is not harmful because VMTs typically do not overstep their training or skill level. An administrator echoed this theme and further described that patients prefer to be seen by VMTs:

"blacks believe that all international people especially people who are not black 
are elite and maybe they're professors and all that. So actually, when they're here [smiles] these patients want to be seen by them. [Laughs] They want to be seen by them so they actually prefer that no for me I'm going to the other Doctor, you understand."

This administrator then explained that he does not feel it negatively impacts him or other staff, when patients prefer to be treated by foreigners. Also, Ugandan trainees perceived 'the muzungu effect' as not being harmful. One intern explained,

"it doesn't affect us in any other way, it just makes me happy to see a child excited of seeing a white person [group laughter], instead I laugh and say, yeah! At least the young child has seen a muzungu physically [group laughter] not in pictures."

Four out of ten nurses interviewed also referred to the 'muzungu effect'. A Tanzanian nurse echoed this view that patients indiscriminately wish to be treated by foreigners, regardless of knowing their role or training level. A phenomenon only described in Rugazi, Uganda was that patient numbers increased at the health care facility when it was known in the community that foreign visitors have come. One nurse described this phenomenon:

"we do get a lot of patients because they know that whites have come, they have come with big knowledge, they are going to treat a variety of so many diseases, they are going to be cured, so they [patients] come with vigor that everything a patient has, will go [laughter]."

A clinical officer in Rugazi also described patient preference to be seen by VMTs rather than himself.

Importantly, the health care workers' perspectives were reflective of how patients perceived foreign visitors because the majority of patients interviewed viewed VMTs positively, enjoyed their interaction with VMTs, and perceived them to be more knowledgeable and respectful than local health care workers. This is illustrated in this Ugandan patient's perspective: 
"When we are coming we do not feel lazy, that I'm going there to sit, I suffer... I'm, no, even when you are coming you feel all happy because you know that they [foreigner] are not going to mistreat you, they will not talk to you rudely."

This patient perceived that waiting times are improved when foreign visitors are present at the health care facility. Also, in the Tanzanian patient and family member focus group 'the muzungu effect' is prominent. A Tanzanian patient felt that VMTs explained the patient's condition more willingly and patiently than local health care workers:

"They [VMTs] are very careful with what they are doing because after work they tell you straight out that: you have this disease, unlike our fellows when they tell you once, if you keep on asking for the second time, they react negatively/they become rude."

Even though many participants perceived that patients viewed VMTs or foreign visitors as more knowledgeable than local health care workers, none of them described this as concerning. Patients perceived foreign visitors as the 'white saviour' come to provide aid and overall more empathy and knowledge than local health care workers.

\section{Recommendations for Improvement}

All participants were asked several questions regarding how they would like the process of GHEs improved, how the University of Calgary can improve the host stakeholders' experience, and how GHEs can be more equitable. The main theme mentioned by most host stakeholder groups was the need for bidirectional exchanges to move towards greater reciprocity. Other recommendations included improving the process of GHEs through better communication and establishment of a host international relations office. Recommendations were provided for the period before VMTs come, during the GHE, and after VMTs return to their home country. 
To improve the process of GHEs as well as improve reciprocity, participants discussed the need for purposeful collaboration between HIC and LMIC institutions, not only in regards to GHEs, but also other collaborative activities.

\section{Need for Exchange Opportunities- 'that's obviously not met'.}

Many participants emphatically stated the need for exchange opportunities for LMIC host stakeholders. One Tanzanian administrator and all four Ugandan administrators emphasized the need for exchange opportunities for themselves or local trainees to ensure fairness and mutual benefit. One administrator explained that what host stakeholders learn on exchange could be applied to improve services in their health care facility. Another administrator accentuated the need to build capacity for exchange opportunities for local trainees:

"we have always said if there is an opportunity for our students to go like that's the main thing. It feels bad having your own students not have any exposure at all. It is uh, it is sad. They keep inward looking and then you're thinking, oh, if they went out they would change [...] even if it's a week or two, it changes someone's attitude. So, building the capacity for our students is number one."

Three out of four Tanzanian physicians, all Ugandan physicians and one Ugandan clinical officer strongly advocated for exchange opportunities to a HIC as crucial to improving their own health care services. One physician described how an opportunity to go to a HIC on exchange allowed her to improve services in her own LMIC institution:

"it makes changes when I come back, yeah, like there you find them from the lab they have a full blood picture results within one hour, or even immediately, so here our lab probably in the past they used to have like a week to give you a full blood picture results, which doesn't help the patient so when you come back you will just tell them a full blood picture can be done within an hour so try to do this"

Another physician repeated the need for exchange opportunities for local trainees multiple times 
throughout the interview:

"I will not say they should send us sundries or what, no, exchange. Uh, let them help facilitate some of our undergraduates and postgraduates to go there [...] and also let there be a way of us sending one or two [trainees] for every ten that come. Yes. Yeah, we may not go there to practice but even just observing, changes the way, you know, their outlook of things."

It is interesting, as in this physician's quote, that when a few participants quoted numbers of local trainees they would like to send for exchange, they are fewer than the HIC sends. Participants were not requesting equal one-to-one relationships but similar opportunities.

Not quite as many nurses mentioned exchange opportunities as a suggestion for improving their experiences in regard to GHEs and in making this process mutually beneficial and reciprocal. Of the nurses who did recommend exchange opportunities, two went on exchange to a HIC themselves. Five out of ten nurses stated that exchange opportunities to a HIC should be implemented to ensure fairness. One Ugandan resident explained that if local trainees went to a HIC to learn about continuous positive airway pressure (CPAP) machines they could come back and learn how to make them locally,

"now if we had taken someone from here there and they learn how to do it [manufacture CPAP machines], it would, it would be quicker [...] they [local trainees] know this land better so they can look for those things around better, so in the end, you find that the exposure will not only help that person but they would come and help people back home as well [...] so for it to be an exchange program, a true exchange program, I think is, is really needed and should be considered by both sides..."

None of the community liaison members interviewed mention the need for exchange opportunities, although one community liaison member mentioned that she heard that local trainees who go on exchange to a HIC outperform local trainees who have not and are more hard-working than those who have not gone on an exchange. Patients did not mention the need for exchange opportunities 
for their own health care workers and trainees. Most participants, apart from patients, viewed exchange opportunities for host staff and local trainees as a crucial process to ensure reciprocity. Given the significant benefit to VMTs when going on a GHE, some participants felt that is up to HIC institutions to fund bilateral exchanges. Others felt funding should be the responsibility of their own institution but recognized the difficulty. Participants felt that exchange opportunities to a HIC institution would be of significant benefit in terms of learning, improving patient services, and increasing applicability and sustainability of LMIC projects as local trainees understand the local context better. Participants advocated for an increase in the number of exchange opportunities for host stakeholders as a major step towards reciprocity, even if the number of students being exchanged is not equal.

\section{Improve the Process of GHEs- 'coordination is a keyword'.}

Participants’ suggestions to improve the process of GHEs highlight their concerns regarding the disorganization of GHEs, lack of communication, and lack of preparation of VMTs. Communication is a major theme that was emphasized as crucial throughout the entire organizational process. Other recommendations for improvement range from selection of VMTs and preparation before the GHE, orientation and supervision during the GHE, and assessment and feedback after the GHE.

\section{Communication.}

Improved communication is a theme that recurred many times throughout all participant interviews, except for the patient focus groups. Participants recommended that communication be improved before, during, and after a GHE occurs. For example, a Ugandan administrator 
recommended improved communication regarding what is expected of host supervisors:

"what they can do is as I said, okay yeah that communication comes, but also to give us more clear guidelines on what exactly is expected of the facility when these students are here [...] I want them to tell us that these are maybe medical students, they're interested in this condition, $\mathrm{mm}$, or maybe they're interested in this topic, I, to be basically more specific about what exactly is required of us."

A physician explained that communication should not only be improved between the HIC and

LMIC institution but also at their own institution between the various departments and the International Relations Office:

"I think getting the departments aware beforehand. We've seen some students, I wouldn't want to say dumped! We've seen some students come and you're like where have you come from? You know, so making, umm, keeping the communication open so that people are well aware of when and how many and what level of students are coming, I think that will help them to be able to find a conducive environment. So, I know there's an International relations office now, so they should not just stop there. There should be continued trickling down of information to the user departments where these students are ending up."

Also, nurses emphasized that communication needs to be improved, especially in terms of notice of VMTs arriving. Local trainees, community liaison members, and clinical officers also emphasized the importance of communication. The majority of suggestions were advance communication before VMTs arrive, but one Tanzanian trainee emphasized communication is paramount throughout the process:

"I mean one has to communicate from the beginning to the end. I mean one has to communicate, there should [be] some good information instead of being surprised...”

Within these suggestions, there was an implicit theme that currently host stakeholders on the front lines are not being made aware of the details of VMTs coming, or that perhaps this is not communicated well by host administration. 


\section{International Relations Office.}

A major difference between the Ugandan host site in Mbarara and the Tanzanian host site is that MUST recently established an International Relations Office (IRO) in 2016. This appeared to be positively perceived by all host stakeholders in Mbarara in that it improves the coordination of GHEs. One Ugandan physician explained the benefits of an IRO:

"Fortunately, there is now a new international relations office where most of them should come through, they are organizing... they are kind of reviewing a structure for the process... we have already been in discussions to streamline the whole process, how they come, what they contribute, how much they pay, how they get attended to and all that, so that needs to be organized in time."

The IRO is also a central office where VMT fees are being collected and guidelines are being implemented as to how these funds should be used:

"that percentage of the monies I have told you that students pay, a percentage goes to the pool for MUST students, that's the plan. So, in future if someone has an opportunity to travel, maybe stipend, maybe conference fees, we can use that money to help a student also travel because it's not about receiving people but who's going out."

Although the IRO is still in the process of developing guidelines and standardized processes, host stakeholders viewed it as having great potential for improving the process of GHEs and improving benefits to the host.

In Tanzania, one stakeholder advocated for development of an international coordination desk. Also, based on Tanzanian participant responses, there did not appear to be a standardized process for accepting VMTs. A Tanzanian administrator explained how he is involved in accepting VMTs; however, there appeared to be multiple routes to be accepted for a GHE. He described that VMTs from one HIC institution have stopped coming and that this may be due to a lack of coordination. Based on recommendations for creation of an IRO in Mwanza, Tanzania 
and the positive feedback on the IRO in Mbarara, a theme emerged that an IRO has much potential to improve the GHE process for both VMTs and host stakeholders.

\section{Suggestions before VMTs arrive.}

Recommendations for the time period before VMTs arrive related to selection of VMTs, pre-departure training for VMTs, and preparation of host stakeholders.

\section{Selection of VMTs.}

One recommendation suggested by a few participants, including nurses and trainees, was that there should be screening and selection of which VMTs come for a GHE. A nurse recommended to change the process and explained, "I could say that they need to pick only those who are really interested to come to our setting/environment." One physician explained that selection of VMTs is already done at their institution, "they are screened, we are involved in deciding who comes and whether we can handle them at a time." However, this physician did not elaborate on what selection criteria are used when deciding which VMTs could come. Screening and selection of who and how many VMTs come for a GHE was perceived to be an important consideration in organizing GHEs.

\section{Pre-departure training for VMTs.}

Another recommendation was that VMTs should be better prepared before they arrive. Although some participants described that VMTs are well-prepared, there were also a significant number of participants who recommended improved preparation. A health facility administrator described that those who come within an institutional collaboration are typically well-prepared, but those who come independently are usually not as well-prepared, 
"so some are really well-prepared especially when there is an already existing collaboration you know [...] so the people who have been here before help those who are coming, so like they are prepared mentally and everything but those ones who don't have a pre-arrangement sometimes you see that they really don't know much about Uganda.”

One physician provided a very specific recommendation for preparation to address the lack of emotional self-regulation of VMTs:

"I think there is a need to improve the...sort of psychological preparation of students...um, and you know, we've developed some material around the stages of grief [...] having something like that, a structured psychological preparation that is part of the process, I think that also would be beneficial..."

Nurses also accentuated the importance of preparation prior to arrival. One nurse described that VMTs are fairly well-prepared but need improved preparation in regards to emotional selfregulation. In contrast, all the registrars and clinical officers stated that VMTs are well-prepared. Both community liaison members and patients perceived that VMTs are well-prepared. Descriptions by Tanzanian trainees and Ugandan residents illustrated that they perceived VMTs as well-prepared in terms of learning about the surrounding environment of the host site and learning a few Swahili words. However, Ugandan medical students and interns did not find VMTs well-prepared especially in terms of coming with required personal equipment. Other than carrying hand sanitizer, interns found that VMTs do not wear uniforms as expected, and they don't come with their stethoscope or notebooks. Medical students stated that VMTs are not prepared in terms of interacting with patients. Although quite a few participants perceived VMTs as wellprepared, especially in terms of their general knowledge about the country, many others viewed VMTs as ill-prepared to actively participate in the wards and lacking emotional self-regulation. To remedy this, host stakeholders recommend improved pre-departure training by the sending institution. 
Preparation for host stakeholders.

There were also a few recommendations that host stakeholders need to be prepared before VMTs arrive. One community liaison member advocated for improved training of community health workers who supervise trainees:

"I think what they can do [...] training of health workers who will also supervise students in the communities, so let them equip skills, knowledge and skills with the health workers both at MUST, they can also organize some trainings at the health facility."

A nurse also mentioned the need to prepare a "good environment" for VMTs before they come. Although it is somewhat unclear what exactly host stakeholders wish to prepare before VMTs come, it is clear that they also would like to engage in local preparation.

\section{Recommendations during the GHE.}

Recommendations for the period of the GHE included orientation of VMTs and host stakeholders, specific learning objectives for VMTs, supervision including discussion of whether foreign preceptors should accompany VMTs, incentives for host stakeholders, some specific recommendations by local trainees, and the need to increase the length of GHEs.

\section{Orientation.}

Although not all participants discussed pre-departure preparation for GHEs, there were also many who discussed the need for orientation once VMTs arrive at the host site. One nurse explained that VMTs need to be orientated to the ward: "the other expectation is the issue of orientation, they aren't oriented...they come straight out to the ward, they don't know our environment..." However, one administrator described the orientation process for VMTs in some 
detail including introductions to staff on the ward. Not only should VMTs be orientated, but Ugandan interns described how local trainees should be orientated on how to interact with VMTs:

"At the same time, the students [local trainees] in the ward should be told that these people [VMTs] are of the same caliber with you, so there will be need for orienting, to orient these students to tell them that these people are the same as you, you are of different skins, you are of different places of origin but your objective here are the same, so therefore feel free to interact with them [VMTs], learn from them and let them learn from you. So the whole basic thing is that [...] there should be clear orientation of these people [...] so that they be able to freely and effectively interact for the benefit of the two groups of students."

As part of orientation, local trainees, but especially nurses, recommended that VMTs should be well-introduced. One nurse explained that VMTs are introduced to the host physicians but not to the nurses: "I think introduction is used to be done to the doctors, when they get to the ward they continue with the rounds so there is no more introduction to the nurses as I pointed out." Physicians also described the importance of VMTs introducing themselves to the nurses. Trainees emphasized the importance of introductions to improve interactions between themselves and VMTs. Orientation of both VMTs and local trainees was viewed as important, with formal introductions to all host stakeholders being a major recommendation to improve interactions.

\section{Specific Learning Objectives.}

Unclear learning objectives of VMTs are a significant challenge for host stakeholders.

There were many recommendations that VMTs should come with specific learning objectives or logbooks. One nurse suggested that the sending institution should complete a host site visit to better understand the LMIC context and develop specific learning objectives for VMTs: "so if the university can really... like now they are sending their students to medicine ward, they come and do a survey what do these people [VMTs] really need to learn from here." Local trainees 
emphasized that specific learning objectives are important for local staff to be able to evaluate VMTs. As reiterated here, unclear learning objectives was a challenge that participants’ mentioned many times, and the need for specific learning objectives for VMTs is a strong recommendation by many participants to assist supervision and assessment of VMTs and to ensure VMTs' learning objectives are achieved. Some participants would also like to be aware of these learning objectives before the arrival of VMTs, so that the host can be prepared to help the VMT achieve their objectives.

\section{Supervision.}

Supervision of VMTs is a major challenge faced by host stakeholders as discussed earlier.

There were some recommendations on how to overcome this challenge. Some host stakeholders recommended designation of a local supervisor for each VMT whereas others recommended that a foreign preceptor accompany VMTs. An administrator explained how VMTs are supervised:

"...the responsibility for supervision falls on the head of department but the head of department usually delegates to other staff within the department or within the ward yeah so that is what the department do, and that's what we encourage, we say 'don't leave, these are students you don't leave them alone' so they...they are normally supervised and are attached to a local student yeah...yeah.”

Another administrator placed more of the responsibility for supervision directly on the VMT. He explained that physicians may be on the ward one day and not the next, so it is up to the VMT to remain part of the team. A nurse described that VMTs are supervised by both physicians and nurses. Recommendations for supervision included assigning VMTs to specific mentors, but this may be difficult so VMTs should share responsibility in participating as part of the local team and making sure they are supervised. Lastly physicians, nurses, and local trainees should all be involved in supervising VMTs. 
Foreign preceptors accompanying VMTs on their GHE is utilized at the undergraduate level by the University of Calgary Cumming School of Medicine in an effort to minimize the supervision burden on LMIC institutions. However, the perceptions of host stakeholders on foreign preceptors coming along to supervise VMTs were variable. Although some participants viewed it as having a positive effect, others viewed it as having a negative effect. Some of the benefits of foreign preceptors, as mentioned by participants, included that they help VMTs feel more comfortable, teach local trainees, and assist with patient care. A resident recommended that a foreign preceptor come along with VMTs so that local trainees benefit more: "I think the University should sometimes send along their lecturers, their professors, we could benefit from, you know, from lectures from them with their students, yeah.” Also, interns shared this same perspective but it was not mentioned by Tanzanian trainees or Ugandan medical students. A few nurses recommended that VMTs come with a foreign preceptor. Three physicians viewed foreign preceptors as having positive effect. One physician explained,

"to me the thing [...] is that you need to have faculty...ideally you need to have faculty on the ground with the visiting students...and the goal of that is to prevent the students from detracting too much from local faculty..."

This physician acknowledged that sending a foreign preceptor is not always possible, and therefore there needs to be a designated local preceptor who receives certain benefits from the sending institution.

However, three physicians advised against a foreign preceptor accompanying VMTs. One physician explained:

"Well, they can have them but I would rather that they trust the system here to handle their elective students. Yeah, if need be we can have focal persons who can be oriented by their team to, on how to handle their students. But also, they should have the trust that the University they're sending their students to, will actually have their best interests at heart. Yeah, so foreign preceptors can come but I don't, 
I wouldn't promote that so much."

This physician preferred that the sending institution focused on building capacity so that local staff can supervise students. He felt that sending institutions should trust host physicians to supervise VMTs. Another physician explained that a foreign supervisor would not know the LMIC context well enough and that VMTs should learn how host physicians practice in their resource-constrained environment:

"he has come to see how we do our things in our limited resources so ah why not... why shouldn't you allow me to supervise that candidate? I mean in my resources... in the way I do it."

An administrator explained how there are both advantages and disadvantages to having a foreign preceptor come along:

"Yeah, the negative effect is mm, especially on us the health workers, when we see that maybe they have come with a Professor, we assume that maybe we want to do things the right way. You understand what I'm saying, so somehow, we're timid, we become timid. We know that aha this is a Professor now, what if they see me doing this one wrong, or what if they see me doing this one the way it's not supposed to be done, that's the only negative bit. But on the other part is, on the other side, they teach and they also teach us."

The overall impact of a foreign preceptor accompanying VMTs is equivocal as some stakeholders perceived it positively, whereas others viewed it negatively. Ultimately, it does not appear to be a perfect solution in minimizing burden on host institutions and host stakeholders.

\section{Incentives.}

A few stakeholders recommended that incentives be provided to host stakeholders to improve the process of GHEs. Some recommended incentives as recognition of their dedication to teaching VMTs, others as remuneration for their time, or as motivation to teach VMTs. Both financial and non-financial incentives were mentioned. An administrator (who is also a physician) 
highly recommended recognition certificates as an incentive for themselves and other health care staff:

"just by recognition, maybe if they could give certificates to the, to those health workers, it would be very good because usually when they come, okay the students give those small, small things, but also something which shows that really I've been involved in this program. Like me I've been involved in this program for so many years, but I have not even got a certificate really to show that I have helped students. So, if they could come up with those motivation avenues for us even to get more motivated to interact more with the students it would be good [...] recognition by giving certificates and maybe medals to the health workers or even the facilities which have participated in this program, $\mathrm{mm}$."

A clinical officer mentioned a challenge related to financial remuneration. He explained it is well-known that the administrator receives facilitation (money) from VMTs who come; however, this is not always given to those involved in teaching VMTs. He recommended that this facilitation be given to those involved in teaching VMTs. Several stakeholders recommended some financial remuneration for those who supervise VMTs. Nurses, local trainees, and community liaison members did not mention the need for incentives. Both Ugandan and Tanzanian physicians and administrators viewed incentives as a recommendation to be seriously considered by sending institutions to recognize their efforts in teaching VMTs and as a way to relieve the burden of supervising VMTs.

\section{Local trainee suggestions.}

A few themes identified were strongly recommended mostly by local trainees. One of these recommendations was that VMTs' schedules should be synchronized with that of local trainees. Local trainees expressed a desire that VMTs be treated similar to themselves during a GHE, in terms of work hours, evaluation, or interaction with host physicians. As one Ugandan resident explained, increasing the time VMTs interact with local trainees will also increase mutual 
benefit. Ugandan medical students also suggested VMTs follow a similar schedule to local trainees as often they only come for part of the rounds. Local trainees would like VMTs to be immersed in all aspects, including call:

"Like I have told you they can come for one hour, like he has also mentioned it, and they go away. So, this still applies also on weekends, I have not seen them in the weekend, I have never seen any on call and yet these are the best times, opportunities that they can use to learn."

Pairing of VMTs with local trainees was recommended multiple times by local trainees. A resident emphasized that pairing VMTs with local trainees would minimize the burden and enhance learning. One resident emphasized he would like VMTs to be treated equally as local trainees are treated:

"I think we already mentioned that we would like to change. [Laughter] We would like them to, to not be treated so specially. They're special because they're visitors but we would like for them to be treated almost as equal, almost equal to the, to the local residents and [...] undergraduates."

Evaluation of VMTs was viewed as important by local trainees to ensure VMTs are treated similarly to local trainees: "I don't know how they are evaluated or how they account for their time in a hospital [...] to be fair no one evaluates them for doing anything." Local trainees were quite adamant that VMTs should be immersed in clinical work and evaluation of their learning in the same way as local trainees.

Increase the length and continue GHEs.

Host stakeholders unanimously advocated for increasing the length of GHEs as they find that often they are too short. There was no declaration made by the participants as to what length of time constituted too short a time. Increasing the length of GHEs was thought to improve the interaction with host staff: “maybe give them more time because if you're working in a very 
limited uh, in a very limited space of time you may not have the liberty to interact with, the way we want them to interact." Another physician explained that often GHEs are too short for VMTs to meaningfully engage in a research project and therefore length of a GHE is very important as to what objectives can be accomplished.

However, no participant recommended that GHEs be discontinued but recommended that they continue with ongoing improvements to the process. One physician advocated for GHEs to continue as there are also many benefits to local trainees. Another physician explained GHEs should continue as medicine is continually changing:

"My expectation I think they should continue to come...it is an obligation, this should continue because students keep on changing, medicine keep on changing so it is better to continue with it."

All participants recommended that the length of GHEs should be increased and they would like GHEs to continue.

\section{Participant recommendations after VMTs leave the LMIC host site.}

Host stakeholders also provided recommendations as to what should occur when VMTs return to their home country. Recommendations included: 1) feedback by VMTs and sending institutions and, 2) recommendations for long-term benefits through ongoing communication and return of VMTs to the host site once they are finished their training.

Feedback- 'it would be nice to get feedback'.

Host stakeholders viewed feedback as important for both the VMTs and for themselves.

One nurse explained the importance of feedback to improve the learning experience of VMTs:

"And also once they come and they've gone back, let them continue the communication. Okay, let them know, let us know how have they benefitted themselves, [emphasizes by tapping on the table lightly] what areas do we need to 
improve on [...] How can they advise us to improve better, to make, to make their learning, okay, conducive."

A physician advocated for regular feedback throughout the GHE through regular meetings and a final report. Another administrator would like ongoing feedback to find out about the career progress of VMTs:

"I think we should keep track of the students who are... who have come to Mbarara and see how they have progressed in their career and how their coming to Mbarara contributed to the progress in their career [...] it would be good so that every year we can send [a] questionnaire and say 'how are you this year, so what are your achievements, do you think your coming to Mbarara contributed anything?'”

Interestingly, local trainees did not mention that they would like feedback from VMTs about their experiences.

Recommendations for long-term benefits- 'relationships that may not just stop here'.

Several host stakeholders recommended that GHEs should have some long-term benefit, with some recommending ongoing communication but others emphasizing the need for VMTs to return once fully-trained. A physician explained how he expects ongoing communication and relationships once VMTs leave: "we expect them to uh, I mean make personal friendships and relationships that may not just stop here but even continue when they're away from here." An administrator explained how to make GHEs sustainable:

"My major expectation of these medical trainees is they should not come to Mbarara once and go back forever, my expectation is they come to Mbarara, they establish networks with our students and even staff and then something grows out of that collaboration, so they come as medical students, they go back, they finish their studies but eventually they come back either as researchers or as teachers and collaborators so that from every university where a student comes from something should...something tangible should come out of it, to me if that happens that will be a very, very big success and one which is sustainable." 
Host stakeholders strongly advocated that GHEs should not just be an isolated experience, but that there is a need for GHEs to lead to long-term benefits through ongoing communication and return of VMTs to the host site once they are full-trained.

\section{Collaboration to achieve reciprocity.}

Although there were many different recommendations by host stakeholders to improve the process of GHEs, a main theme is that HIC institutions and LMIC institutions need to work together, both on GHEs and other collaborations, to achieve mutual benefit and reciprocity. Many host stakeholders would like GHEs to continue but acknowledged that the process needs to be improved through better and purposeful collaboration. An administrator described this need for purposeful collaboration:

"well, I think if the University of Calgary wanted to improve the ah learning conditions here I think like the way others have done is that they should go ahead to sign an MOU with them and then we agree on a range of issues, where they can contribute and where we can contribute so that a mutually agreed position is... is... is reached."

One physician recommended for improved collaboration between all institutions to standardize the process of GHEs:

"the sort of structure of rotations, the orientation for rotations...I think is one thing that could definitely be done to improve and...and...that would require better coordination between different u...different foreign institutions, so that, for example [name of US university] collaborating with Calgary collaborating with um, [name of German university], all of these universities collaborating together to standardize programs...”

Nurses mentioned that through collaborations facilitating GHEs, HIC institutions should also provide physical resources. Many nurses also requested collaboration on continuing medical education workshops by foreign visitors to enhance mutual benefit. Participants recommended 
that they would like collaboration on teaching both from VMTs and visiting lecturers.

Research collaboration was another theme that host stakeholders emphasized. One administrator referred to the benefits of research collaborations when discussing GHEs:

"their presence can help us improve in some of the areas for example we have some relationship with [name of German university] and there are some big studies that have been done in collaboration with [name of German university].”

Although as earlier described, VMTs themselves may provide little benefit, the collaborations developed as a result of GHEs have increased reciprocity. A physician emphasized the importance of a memorandum of understanding (MOU) to prevent redundancy in collaborations:

"each department ideally should have an MOU... you know, that stipulates how and in which areas they're going to collaborate or partner in. Yeah, and so, like for Obs/Gyn, ours is quite clear. If we're to collaborate with you, we say we're going to collaborate with you on resident training. Or on this area, so that when another collaborator comes, we say no, this one is covered. Is there another area you want to collaborate with or can you synergize the efforts of this collaborator you found here? Hmm.”

The reciprocity and mutual benefit of GHEs are judged by host stakeholders, not on their own, but whether GHEs have or will result in a successful collaboration including other endeavors, such as research, capacity-building through physical resources, training of host staff, and exchange opportunities for local trainees.

\section{Summary}

The host stakeholders who participated in these semi-structured interviews and focus group discussions were able to provide their individual perspectives openly and reflectively as they discussed the complexity of the phenomenon of GHEs. All participants are familiar with GHEs as a commonly experienced, yet indistinct, phenomenon as they often contend with many 
foreign visitors in different roles. Participants listed a variety of benefits that they gain because of VMTs coming to their site, but this appeared to be the silver lining of a phenomenon that has significant challenges and burdens. A few of the participants were quite dissatisfied with the current process. Interestingly, many participants described significant challenges and harms while denying harms when asked directly. All of the challenges reported support a strong theme that GHEs are a one-sided relationship as host stakeholders minimally benefit. Some ethical challenges are identified, not from the host stakeholders' perspective but from a HIC perspective regarding the 'muzungu effect' and that VMTs are perceived to come to LMIC to 'touch patients'. Patients are the only host stakeholder group to not identify any harms and they do not view the relationship as one-sided. Despite the multitude of challenges and harms, host stakeholders would like GHEs to continue, with the hope that their recommendations will be implemented to promote mutually beneficial and reciprocal GHEs. 


\section{CHAPTER 5: DISCUSSION}

\section{Introduction}

The purpose of this study was to explore the perspectives of host stakeholders in Tanzania and Uganda in relation to the phenomenon of global health electives (GHEs). Although there are few studies that have focused on host stakeholder perspectives on global health electives, these limited qualitative studies provide rich, contextual details that help to situate the findings of this study. This chapter will highlight the major findings of this study, explore how the themes intersect with existing literature, critically appraise the research findings and suggest how findings might translate into practice. I will review the ethics and best practice guidelines for GHEs developed by the Working Group on Ethics Guidelines for Global Health Training (WEIGHT) in light of my findings, which provide new evidence, and provide practical suggestions using the WEIGHT guidelines as a framework. (Crump et al., 2010) Deliberating implications for research, I discuss areas of future research to further understand the phenomenon of global health electives. I will also acknowledge and discuss limitations of this study. My study findings suggest that the best way to work towards mutually beneficial and reciprocal GHEs, they should occur between collaborating institutions with a bidirectional focus.

\section{Overview of Findings}

I clustered emergent themes identified from the participants’ accounts around three superordinate themes: 1) GHEs are a common, yet indistinct phenomenon; 2) Challenges and burdens of GHEs; and 3) Recommendations for improvement. Through interpretive analysis, I found connections between many emergent themes and sub-themes both within and between 
participant' accounts which provide evidence for the superordinate themes (Figure 2).

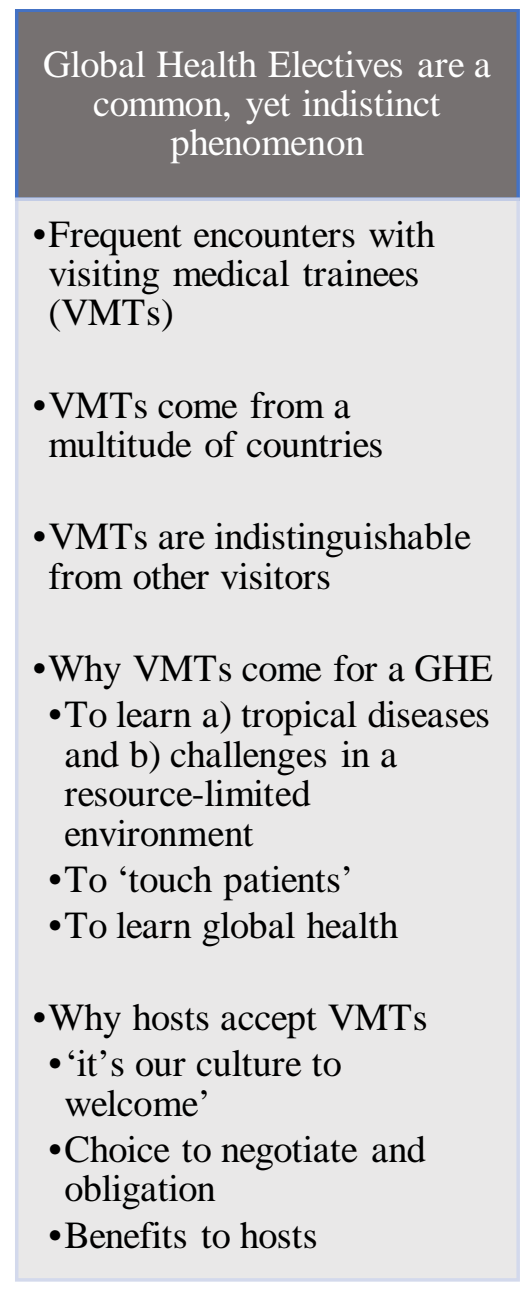
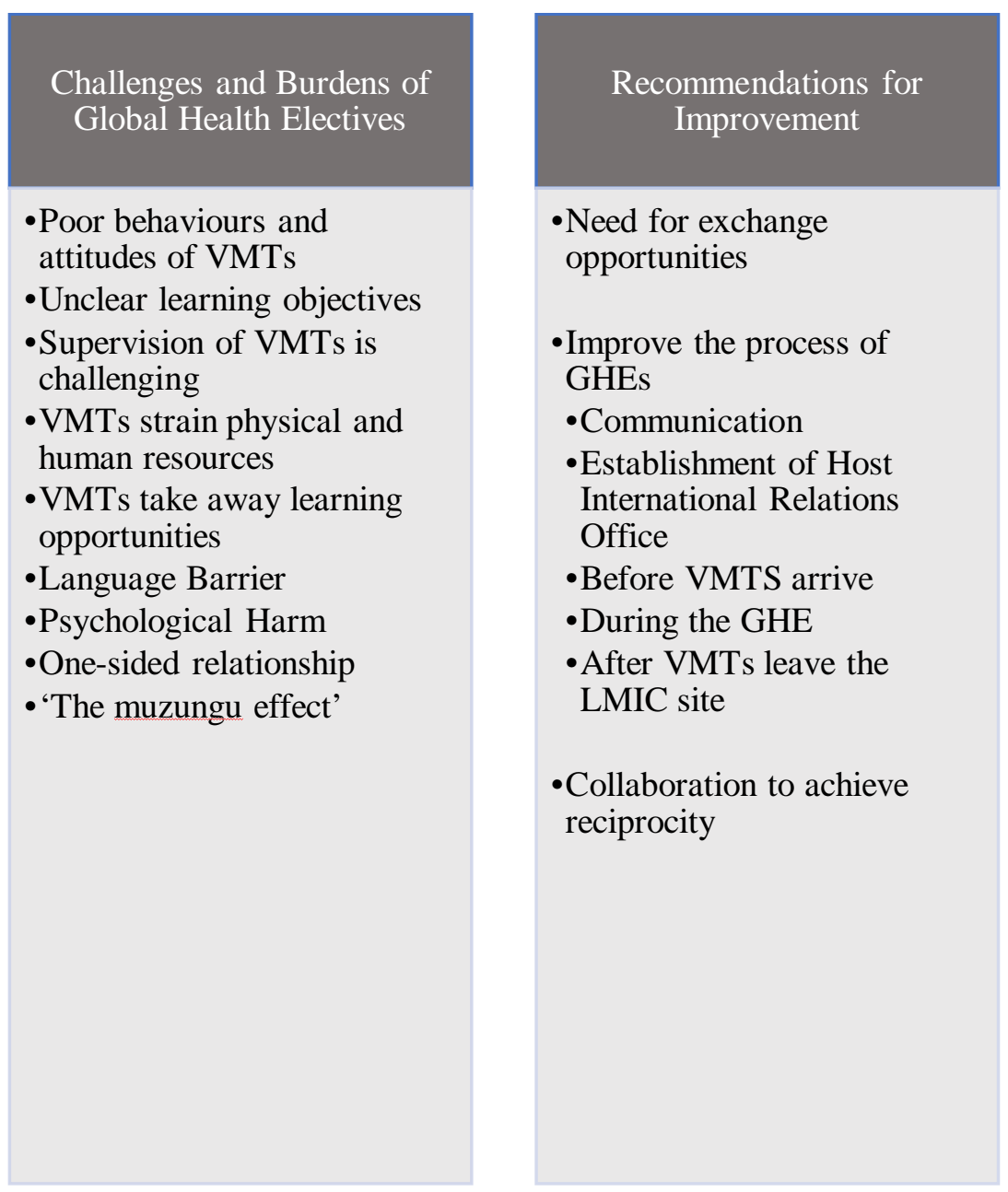

Figure 2. List of Superordinate and Emergent Themes

I identified a few significant differences between perspectives based on the host site, but overall, I found similar perspectives across all host sites. I observed two thematic differences between Tanzania and Uganda. Language appeared to be a more significant barrier to communication and interaction in Tanzania, compared to Uganda. This difference is likely because Swahili is the official language in Tanzania, and although physicians conduct hospital rounds in English, local staff often revert to using Swahili based on my observations. In comparison, English is the 
official language in Uganda, and although many patients do not speak English, all hospital staff are fluent in English. The second difference was that the host site in Mbarara, Uganda has an international relations office (IRO) while the host site in Mwanza, Tanzania did not. The presence of an international relations office appeared to contribute to greater understanding of the process of GHEs in Mbarara compared to Mwanza and participants in Mbarara viewed the IRO as an important step in improving the process of GHEs. I found a few differences in emergent themes between the rural site, Rugazi Health Centre IV in Uganda, and the two urban host sites in Mwanza, Tanzania and Mbarara, Uganda. At the rural site, participants were overall more positive about the benefits of GHEs and mentioned fewer challenges. Also, small research or quality improvement projects by visiting medical trainees (VMTs) are perceived to have greater value at the rural site as compared to the urban sites. Despite these few differences, a common theme runs through the majority of participants’ perspectives: GHEs are a commonly occurring experience with a variety of benefits, but there are significant challenges and burdens which need to be addressed to promote equity and mutual benefit in GHEs. I will further describe how my study findings intersect with the existing literature.

\section{Intersections with existing literature}

\section{GHEs are a common, yet indistinct phenomenon.}

Low-and middle-income country (LMIC) host institutions may receive VMTs, as well as many other international visitors in different roles; yet, this is a theme that is rarely discussed in the literature regarding host stakeholder perspectives on GHEs. However, GHEs are a wellestablished phenomenon as LMIC host institutions have received VMTs since at least 1968 for international educational rotations. (Waddell, 1976) Not only have they received VMTs for over 
50 years, but also high school students, undergraduate students, graduate students, researchers, and fully-trained professionals in a multitude of disciplines, who travel for variable lengths of time to health-care settings in LMIC. (Macfarlane et al., 2008; Sullivan, 2018) International visitors come within the context of a formal institutional partnership or on an ad hoc basis. These international visitors come either as learners or professionals as part of an academic institution's global health program, research studies, medical missions, volunteerism or for a host of other reasons. Sykes et al. (2014) in his review of publications discussing short-term medical service trips found forty-five different terms used in the literature for this phenomenon. It is vital to understand that VMTs take part in GHEs within this complex milieu and although, in my study, participants were aware that there are visitors who come as learners, this degree of awareness was variable. I found that although host stakeholders such as administrators, physicians, and local trainees were quite aware that VMTs come to learn, still their perspectives are an amalgamation of experiences with all these international visitors. Despite the interview moderator explaining to each participant the study definition of visiting medical trainee prior to the interview or focus group, participant' perspectives related to a range of international visitors. When VMTs come without clear learning objectives or learning objectives that are not wellcommunicated, they are even more likely to be perceived as another visitor among many. This anonymity of VMTs among the collective of international visitors is important to be aware of as a limitation of this study and previous studies reporting host stakeholder perspectives on a specific group of visitors. (Bozinoff et al., 2014; Kumwenda et al., 2015; Kung et al., 2016) Furthermore, this is something that future studies should take into consideration when attempting to obtain host stakeholder perspectives on a specific group of visitors. Arguably, this theme that VMTs are indistinguishable from other visitors is likely not unique to LMIC host sites but may 
also occur at high-income country (HIC) health care facilities. Nevertheless, language and cultural barriers, limited supervision, and large numbers of VMTs at LMIC host sites would seem to increase the potential impact of this lack of distinctiveness thereby magnifying the probability of causing harm and diluting benefits. This theme of indistinctiveness within a phenomenon that occurs frequently is vital to explore and address.

Only two previous studies on host stakeholder perspectives mention this lack of distinctiveness of visitor roles. Lukolyo et al. (2016) observed that host stakeholders might have difficulty differentiating between visiting medical students and residents and therefore did not ask participants to differentiate when sharing their perspectives. DeCamp et al. (2014) observed that many patients receiving care from an American NGO were not aware of the name of the group providing services despite a long-term relationship between the NGO and the community. The authors speculated that this might be because individuals learn about the medical team's presence through word of mouth or access to care could be so poor that recipients focus on 'what' more than 'who'. I found that patients in my study were also unclear about the role of the visitor; however, I found this confusion also applied to other host stakeholders. Other studies focused only on medical students or simply referred to medical trainees but did not elaborate further on training levels. (Bozinoff et al., 2014; Kumwenda et al., 2015; Kung et al., 2016) None of these studies mentioned that they might have obtained mixed perspectives on other trainees, such as nursing trainees or high school students. However, it was clear from my study that host stakeholders may discuss perspectives related to a variety of visitors and not only VMTs. Occasionally I was able to distinguish between participants' expectations of medical students versus residents, but most of the time it was impossible to distinguish which perspective related to which visitor role. Many times, participants referred to 'these people', 'these whites', 
'foreigners', or 'students'. Therefore, this indistinctiveness is both a limitation of our study in obtaining perspectives only related to VMTs but also a challenge that high-income country (HIC) and LMIC institutions should recognize. This theme suggests that GHEs should be developed as part of structured, collaborative partnerships between HIC and LMIC academic institutions rather than allowing VMTs to arrange their placements at any LMIC host institution. Within a structured collaboration, the evidence suggests that improved communication and specific learning objectives would help clarify the role of VMTs which may minimize harms and unintended consequences.

\section{VMTs come to 'touch patients': an ethical concern.}

There was a common understanding by host stakeholders that VMTs come to 'touch patients'. It is an ethical concern that most host stakeholders perceived VMTs seek to participate in GHEs so that they have an opportunity for hands-on engagement with patients which they are not allowed to do in their own countries, even if VMTs are not participating beyond their skill and training levels. I found a lack of understanding amongst host stakeholders at what level VMTs are allowed to engage with patients at their home institution. Within my study, I also found that host stakeholders did not mention that VMTs are practicing on patients beyond their capabilities; however, other authors document examples of this occurring. (Elit et al., 2011; Shah \& Wu, 2008a; Sullivan, 2018) Despite this perception that VMTs come for the opportunity to 'touch patients', it is likely not the intent or objective of sending institutions for VMTs to obtain greater levels of engagement with patients in LMIC than they would be allowed to have in their home institution. My findings suggest a need to discuss and clarify with LMIC host stakeholders the extent to which VMTs engage with patients in their home institution in comparison to the 
host institution.

However, this ethical concern does not mean that VMTs should not interact with patients at the host site at all. Although many host stakeholders perceived that VMTs come to their institution for the opportunity of hands-on engagement with patients, some participants expressed disappointment that VMTs merely observed and did not touch patients. These participants were uncertain as to why VMTs did not interact with patients, whether it was a language barrier, perceived racism, or fear of infectious diseases. This issue was also identified by Kung et al. (2016) who found that some VMTs are hesitant to touch patients and appear apathetic during teaching. However, as the author further discussed, increasingly stringent standards call for pre-health students to observe only and for all students to avoid practicing beyond their level of training. Host stakeholders may not be aware of these changing standards or recommendations. These two contradictory themes of 'touching patients' and 'fearing to interact' are challenges which need to be discussed between collaborating institutions to develop a common understanding as to the purpose of GHEs and what is an appropriate level of VMT interaction with patients. Also, it would seem that a policy that promotes LMIC medical trainees to have equal opportunity to engage with patients based on their capabilities when they participate in a HIC elective would be appropriate. This inequity between VMTs and LMIC medical trainees' opportunity to interact with patients when on an international elective is a significant challenge as discussed by Pitt et al. (2016) in their description of a bidirectional exchange program, as institutional regulatory challenges necessitated that visiting LMIC residents only observe patient care during clinical rotations. To address this significant ethical concern, sending institutions and host institutions need to work together to ensure that the level of training of VMTs are well understood by host staff, VMT learning objectives are clear, and 
VMT and LMIC medical trainee engagement with patients is appropriate in both HIC and LMIC institutions to their level of experience, their level of training, and the available supervision.

\section{VMTs come to learn 'global health'.}

I found a disconnect between participants' understanding of global health and the definition of global health. A few participants perceived that a global health elective (GHE) is a high-income country (HIC) academic institutional requirement for VMTs to complete their training. Although I cannot speak on behalf of all HIC academic institutions, most offer GHEs as an optional experience. (Edwards, Piachaud, Rowson, \& Miranda, 2004) Within this theme of learning 'global health', other physicians in my study viewed hosting VMTs as an opportunity for local trainees to learn about foreign health care indirectly. I found differences in how achieving global health competencies applies to VMTs as compared to local trainees. For example, some physicians and administrators perceived that learning 'global health' is something done by HIC trainees, leading to a misinterpretation that learning ‘global health’ requires international travel. Host stakeholders were likely unaware that VMTs could also participate in electives or rotations in their home country within resource-limited environments or with underserved populations; for example, within indigenous communities, inner-city clinics, immigrant health, and rural and remote electives, given the growing trend of 'global is local'. (Maley, Worley, \& Dent, 2009; Moskowitz, Glasco, Johnson, \& Wang, 2006) In my study, participants did not discuss that local trainees are able to learn global health competencies within their own country except for learning indirectly from VMTs sharing their knowledge of foreign health care systems. Therefore, both VMTs and sending institutions need to be clear about their reasons for choosing GHE host sites, in particular that 'international exposure' is not sufficient 
reason in and of itself. Host physicians also viewed teaching VMTs as an obligation or moral duty to positively impact the practices of global health themselves; in that, through teaching VMTs they will help VMTs care for other patients around the world, for example, emigrants from the host's country. Through these various perspectives on global health, it is likely that there is a vague and varying impression of what global health entails. Host stakeholders often perceived that VMTs are seeking to become 'international doctors'. Kung et al. (2016) also documented a similar host physician perspective that VMTs need international exposure to become 'world leaders.' These perspectives on global health highlight the inequitable and paradoxical perspectives that HIC institutions have unintentionally embedded within host stakeholders' views of global health in that VMTs learn global health by participating in a GHE, but local trainees should try to obtain the same global health education indirectly through VMTs. Global health appears to be perceived by host stakeholders as something that HIC institutions 'do', and yet based on the definition of global health it is "[an] area of study, research, and practice that places a priority on improving health and achieving equity in health for all people worldwide." (Koplan et al., 2009) HIC and LMIC stakeholders are equally involved in this endeavor as it not only involves work between countries, but also within each country. Despite this, participants in my study did not perceive themselves as equal partners in the practices of global health but rather viewed themselves as indirectly involved by helping VMTs achieve global health competencies. As Koplan (2009) further explained, the term global health "runs parallel to a shift in philosophy and attitude that emphasises the mutuality of real partnership, a pooling of experience and knowledge, and a two-way flow between developed and developing countries.” The findings in my study of the perspectives on 'global health' begs the question whether Koplan's definition is an ideal HIC definition that in its' practices are perceived as 
something very different by our LMIC partners. It is imperative that through GHEs, HIC academic institutions do not reinforce the health inequities and learning opportunity inequities that we seek to alleviate, as the perspectives identified in this study suggest that this reinforcement of inequity may occur. Also, this theme serves as a caution for sending institutions that although VMTs are encouraged to develop global health competencies through GHEs, they should not reinforce the perspective that only HIC trainees need to learn 'global health' but that this is something that ideally all HIC and LMIC medical trainees should learn.

\section{Choice to Negotiate.}

Although most health facility administrators did not feel compelled to accept VMTs to come to their host institution, there was a concerning theme regarding lack of choice to supervise as voiced by physicians and some residents. The concern was mainly around VMTs coming at inopportune times, too many VMTs coming at the same time, and feeling pressure to supervise when already overwhelmed teaching local trainees and caring for patients. This concern was also brought to light in Kumwenda et al. 's (2015) study as a participant described that sometimes they have many VMTs coming at one time and therefore VMTs receive a passive learning experience. This concern is further evidence that GHEs should take place in structured, reciprocal institutional collaborations that are transparent and regularly evaluated by seeking input from multiple stakeholders that includes host preceptors and local trainees.

\section{Benefits to Host Stakeholders.}

Many benefits of GHEs to host stakeholders described in my study, were already welldescribed in the literature. Kumwenda et al. (2015) found that although participants were 
realistic about the amount of benefit VMTs provided, mature and responsible VMTs could provide some service, financial contributions by VMTs were beneficial, and they mentioned the mutual benefit of knowledge sharing. Other studies on host stakeholder perspectives also identified themes similar to those in my study, such as a rise in local prestige, resource enhancement, VMTs providing a good example to local trainees, and benefit due to collaborations and bidirectional exchanges. (Bozinoff et al., 2014; Evans et al., 2017; Kung et al., 2016; Lukolyo et al., 2016) No new themes were identified regarding benefits for host stakeholders although further evidence has been provided by my study to support these themes. However, what is unique about my study is that significant challenges were discussed by host stakeholders which have been inadequately or not at all described in previous studies.

\section{Poor interactions with local trainees.}

Although many physicians, administrators, and nurses who participated in this study perceived that VMTs interact well with local trainees and that local trainees derive significant benefit through sharing of knowledge and experiences with VMTs, local trainees’ perspectives contradict these views. Our findings suggested that although local trainees described some personal benefits of interacting with VMTs, the benefits are minimal. This theme reinforces and provides further explanation of the findings of Elobu et al.'s (2014) cross-sectional descriptive survey evaluating Ugandan surgery and anaesthesia trainee perspectives on international global health collaborations. Overall, the authors found that visiting faculty were perceived to improve LMIC residents’ training experience more than visiting trainees. However, given this was a survey, the authors could only speculate as to why this perception existed. I identified that local trainees were particularly critical of VMTs sharing knowledge that is not applicable in their 
context. This perspective indicates a need for cautioning of VMTs in what teaching activities they participate in and a need to closely work together with local medical trainees to determine what would be most relevant in terms of sharing knowledge.

\section{Challenges and Burdens of GHEs.}

Similar to benefits, some of the challenges and burdens described in my study are also previously described in other qualitative studies. Issues around cultural competency and inadequate emotional self-regulation of VMTs are well-described, as well as the perspective that some VMTs come with an arrogant attitude. (Kumwenda et al., 2015; Lukolyo et al., 2016) Furthermore, both Underwood et al. (2016) and Kung et al. (2016) explained how trainees showed lack of cultural sensitivity by rejecting hospitality offerings of food and that this could reinforce negative stereotypes within the mind of the host. My study highlights that cultural insensitivity can take many different forms and may not always be apparent to the VMT; for example, participants reported that use of hand sanitizer by VMTs reinforces negative stereotypes within the mind of patients that they are considered dirty. The theme that some participants perceived VMTs as medical tourists because of their attitudes and actions is a strong theme in several qualitative studies on host stakeholder perspectives. (Kumwenda et al., 2015; Kung et al., 2016; Lukolyo et al., 2016) The literature does not describe host stakeholders identifying the harm of VMTs using blogs or social media to document their experiences, but other literature discusses this harm. (Crump et al., 2010; Frischtak \& Sinha, 2013) Also, other studies reported the challenge of unclear learning objectives and how this makes supervision by hosts challenging. Bozinoff et al. (2014) related that host supervisors wanted improved communication in regards to the student's goals and expectations of the elective. Kumwenda et 
al. (2015) explained that lack of communication led participants to question the objective and relevance of GHEs. Regarding supervision, the authors reported participants felt that VMTs were supervised and not allowed to do procedures they were not qualified to do. This finding is similar to my study, where most participants perceived that supervision of VMTs is important and most often they are supervised, but they acknowledge supervision of VMTs becomes a burden.

The perspective that GHEs are a one-sided relationship due to limited benefit and significant challenges and burdens for host stakeholders is also previously reported. In Lukolyo et al. 's (2016) study, participants viewed the relationship as inequitable because VMTs are not at the host site long enough to understand the system and culture and thereby are unable to contribute much to the host institution. The authors stated that multiple participants felt that longer GHEs would allow for a mutually beneficial experience. In Kung et al. 's study (2016), GHEs were viewed as a one-sided relationship as VMTs 'just come and go' and make promises they do not fulfill. Furthermore, VMTs were perceived not to share research results with host collaborators and host stakeholders did not have equal opportunities to travel to HIC. I identified similar themes where participants advocated that VMTs stay longer, return in the future, and build mutually beneficial research collaborations. Participants in my study identified research as a skewed relationship concerning ethics approval, authorship, and applicability. Elobu et al. (2014) also identified concerns regarding research collaborations amongst LMIC surgery and anaesthesia trainees as many participants found research undertaken by visiting international groups not to be in high priority areas for Uganda, not to have appropriate level of local collaboration, and no trainees who had participated in these research collaborations had published as a coauthor. Interestingly, in these qualitative studies on host stakeholder 
perspectives, there was not a significant theme regarding a need for bidirectional exchange. For example, in Kumwenda et al. 's (2015) study, participants considered various ways through which electives could become more equitable, but they did not mention bi-directional exchange. Kung et al. (2016) reported that several host stakeholders would like the opportunity to travel to the United States. Only in Bozinoff et al. 's (2014) study did participants advocate for formalized bidirectional exchange. In contrast, I found a strong theme regarding the need for bidirectional exchanges, especially voiced by host physicians and local trainees, but also by other host stakeholders. Overwhelmingly, existing literature supports the conclusion that although GHEs provide a variety of benefits to host individuals and institutions, host stakeholders predominantly viewed GHEs as a one-sided relationship with many advocating for various ways to increase reciprocity and mutual benefit.

I identified two themes inadequately described in the existing literature: VMTs take away learning opportunities from local trainees and 'the muzungu effect.' The theme that VMTs take away learning opportunities from local trainees is not well-described in previous qualitative studies. A host preceptor in Kumwenda et al.' s (2015) study explained that local trainees are, in fact, prioritized above VMTs. Bozinoff et al. (2014) explained that VMTs are an increased workload for mentors and thereby can limit opportunities for local trainees. However, in my study, it is clearly described by local trainees that host staff may prioritize VMTs over local trainees, VMTs may intimidate local trainees thereby making them more hesitant to interact during rounds, and VMTs take away operative time from local trainees.

A significant ethical concern I identified is 'the muzungu effect' which relates to patient' views that VMTs are more knowledgeable than local health care providers. This concern has not previously been well-explained in the literature and my findings provide concrete evidence that 
this is a significant effect. Bozinoff et al. (2014) questioned whether patients perceived care offered by foreigners to be superior to that delivered by local staff based on the perspectives of host supervisors. The authors mentioned that this concern merited further understanding. My study is one of very few studies to include patient perspectives on VMTs. A study by Weng et al. (2015) evaluated patient perceptions towards a Taiwanese short-term mobile medical aid mission using a questionnaire survey but this medical mission did not include trainees. The feedback from patients in their study was overwhelmingly positive. Another study by DeCamp et al. (2014) included patient perspectives on trainee involvement (both medical and non-medical trainees) in medical missions; patients described trainee involvement as beneficial to the trainee and to the patients themselves. Patients did not report any negative effects of trainee involvement. The authors did not mention whether trainees were viewed as more knowledgeable than local staff, though perhaps this was because visitors (including HIC trainees) did not work closely with local staff. The authors addressed the concern that patient participants expressed universally positive views of trainee involvement despite concerns about negative impacts of trainee involvement discussed in the literature. They concluded that patients' positive views on trainees was reassuring but that it also reinforced the need for adequate supervision. Based on my study findings, patients perceived VMTs as more knowledgeable than local healthcare providers as described by both patient and physician participants. Patients further described that they often are not told that the visitors are trainees but did not mention any harms or negative effects of VMTs providing care. Yet, this view is a significant ethical concern as this perspective may lead to unintentional, but also unrecognized, patient harm. Finding solutions to address this concern is crucial. 


\section{Recommendations for Improvement.}

Host stakeholders provided recommendations for improvement ranging from the need for exchange opportunities, the need to improve the process of GHEs before, during, and after VMTs are present at the host site, and the need for building collaborations to achieve reciprocity. Several of these recommendations are mentioned in the existing literature. Recommendations identified in other studies included VMTs coming regularly without gaps, increasing length of GHEs, foreign preceptors accompanying VMTs, improved communication, funding from the sending institution to pay for translators and professional training for staff, improved predeparture training, improved guidance from the sending institution on facilitating orientation and introduction of VMTs, and the need for bilateral exchange opportunities. (Bozinoff et al., 2014; Kumwenda et al., 2015; Kung et al., 2016; Lukolyo et al., 2016)

I identified several new themes regarding recommendations for improvement where previous studies inadequately described certain themes. These themes include the need for a host international relations office, foreign preceptors accompanying VMTs, incentives for host preceptors, ongoing communication, and the need for long-term benefit to the host institution. Based on the description of the setting in other studies on host stakeholder perspectives, it was unclear whether there was an international relations office or desk at these host sites. However, in all of these studies, there is a strong theme regarding the need for improved communication and organization of GHEs. I identified that an IRO at one of the host sites was viewed as an essential mechanism to improve the organization of GHEs as well as to improve mutual benefit by collecting fees from VMTs which could then be used to fund local trainees' travel for conferences or a HIC elective. Furthermore, at the site where there was no IRO, a few participants advocated for the creation of an IRO to improve communication and organization of 


\section{GHEs.}

A participant in one study requested foreign preceptors accompanying VMTs, however, this was not further described elsewhere. (Kumwenda et al., 2015) Both Hayward and Li (2017) and Simonelis et al. (2011) described that a HIC supervisor accompanied physiotherapy and occupational therapy trainees; however, they did not investigate the impact of a foreign preceptor accompanying trainees. Intriguingly, my study findings were that the benefit of having a foreign preceptor present at the host site was equivocal. Some participants found that this would minimize the burden on the host site and provide benefit whereas other participants perceived this as a lack of trust in the ability of the host institution to adequately supervise VMTs or not useful as foreign preceptors do not understand the local context.

Incentives for host preceptors were also not previously well-described. Although one study described that host physicians might be unwilling to teach VMTs due to lack of incentives, there was no further description of what type of incentives were requested by host preceptors. (Kumwenda et al., 2015) One recommendation in my study, was for a 'global health certificate' to acknowledge the preceptor's commitment in supervising VMTs. Although financial incentives were also mentioned several times as motivating host preceptors to teach VMTs, some acknowledged that this became problematic when the financial incentives did not reach those supervising VMTs. Therefore, although there is a need for acknowledgement of the time host preceptors take to teach VMTs through financial remuneration or other tokens of appreciation, careful consideration of what should be provided and transparent procedures to implement this should be discussed between the host and sending institutions.

Based on host stakeholder perspectives previously discussed in existing literature, there has been little discussion regarding a desire for ongoing communication and feedback. Lukolyo 
et al. (2016) mentioned that a respondent suggested VMTs also assess host preceptors.

However, in my study, host stakeholders expressed significant interest in understanding the impact of GHEs and improving the process on their side. Participants recommended that VMTs and sending institutions provide feedback to host individuals and institutions on how the GHE experience could be improved. They also wished to know how the GHE impacted the VMT's career trajectory. Not only do host stakeholders recommend ongoing feedback and communication after the GHE, but they would also like to know the long-term impact of GHEs.

Host stakeholders in a previous study identified that VMTs ‘just come and go’, and this was also a concern expressed in my study. (Kung et al., 2016) However, participants further described that to improve the long-term benefits of GHEs for the host institution and community, VMTs should return once they finish their training. Several host stakeholders were aware of the limitations of achieving mutual benefit within the GHE, as VMTs benefit the most from this unique learning opportunity, but they highly encouraged VMTs to return in the future to increase mutual benefit. In conclusion, my study findings expand on themes identified in the existing literature, but I also highlight new findings not previously described. I will now provide a further critical appraisal of my study findings through the lenses of post-colonialism, social justice, and social accountability.

\section{Critical appraisal of findings and how they might translate to practice}

Examining the study findings through the critical lenses of post-colonialism, social justice, and social accountability is crucial to be able to understand how the findings might translate into practice. Although there are many nuances as to how host stakeholders understand the phenomenon of global health electives, overall participants perceived GHEs as having some 
benefit for host stakeholders, but also many challenges and unintended consequences which are difficult to overcome for various reasons. Why these challenges are difficult, but necessary, to overcome are highlighted by appraising them through the lenses of post-colonialism, social justice, and social accountability.

\section{Post-colonial lens.}

My study findings are situated within a colonial discourse. Both Tanzania and Uganda were colonized by Britain, and received independence in 1961 and 1962, respectively. (Commonwealth, 2018a, 2018b) Post-colonial theory grapples with the legacy of colonial rule. It studies the power and the continued dominance of Western ways of intellectual inquiry and the production of knowledge in the academic, intellectual and cultural spheres of the de-colonized country. (Iwowo, 2014) When applying this lens to my study findings, it is clear from host stakeholder perspectives on GHEs that HIC institutions continue to dominate and have the majority of power within the relationship regarding GHEs, within both the process of GHEs as well as the discourse around GHEs. For example, GHEs characterize a movement of VMTs which is largely from 'North' to 'South'. Furthermore, although host physicians perceived that local trainees benefit from the presence of VMTs, local trainees contradicted this common perspective and illuminated that often they are pushed to the sidelines within this phenomenon. This subtle, and perhaps unintended, exploitation of learning opportunities by VMTs can be considered an aspect of neocolonialism. Easterly (2007) described this shift from colonialism to neocolonialism. He explained "uncivilized” became "underdeveloped" and "savage peoples" became the "third world". He further argued that although there was a shift from racist perspectives towards respect for equality, a paternalistic and coercive strain survived. Sullivan 
(2018), in her post-colonial analysis of international clinical volunteering discussed the problematic postcolonial racialized tropes underlying the marketing, discourses, and practices that make up international clinical volunteering. Although she discussed individuals, including medical students, who travel to LMIC through private volunteer placement organizations as opposed to through HIC academic institution global health programs, her analysis can still be applied to VMTs going on GHEs sanctioned by the sending institution. She referenced Teju Cole’s term 'white saviour industrial complex' which refers to how predominantly white and privileged groups assume they can 'make a difference', without understanding the complexities of context of those being 'helped'. This assumption regarding ability to 'make a difference' is not only from the perspective of white and privileged groups but is also embedded in host stakeholder perspectives when they describe how VMTs share much knowledge or how they are 'a big relief'. I challenge that this host stakeholder perspective is more a result of embedded assumptions related to the 'white saviour' and global health discourse rather than an actual reality, as many challenges, burdens, and unintended consequences emerged from participant' experiences. Furthermore, as Crane (2013) described, “Global Health is haunted by a postcolonial power differential that it continually struggles against”. Within this global health discourse, imaginaries of 'resource-poor' people and places are inherently coded as 'Other' and well-intentioned foreigners can 'make a difference'. (Brada, 2011) This discourse of labelling within the global health sphere is also echoed from the side of host stakeholders, most especially patients, as is evident in my findings where VMTs are referred to as 'these whites' who come 'with a good heart'. Sullivan identified two prevalent post-colonial racialized tropes: 1) Volunteers conceptualised themselves as affiliated with a 'global medical imaginary'-or with what they saw as 'real medicine'-yet understood Tanzanian practitioners as lacking sufficient 
professional status to merit full recognition as professionals; and 2) Volunteers envisioned patients as 'suffering strangers' thus justifying their providing sub-standard medicine 'good enough for the poor'. These post-colonial racialized tropes are not only evident from the perspectives of international clinical volunteers, but my findings illustrate that these postcolonial racialized tropes are embedded in some host stakeholder views' that VMTs 'are a big relief' and patients' views that VMTs are more knowledgeable than local health care providers. As Sullivan described, the discourse around global health has uncomfortable neocolonial echoes which also come to light in my study findings. The theme that some VMTs come with an arrogant attitude provides further evidence that these post-colonial racialized tropes exist. Therefore, as I make recommendations from my study findings it is crucial that I acknowledge this post-colonial power differential which is also entrenched in global health discourse and attempt to promote equitable relationships by suggesting that power be equally distributed in the relationship dynamics within GHEs. Host stakeholder perspectives should not be cast into the periphery. HIC views of the significant benefits of GHEs for VMTs should not be magnified to promote GHEs and thereby ignore or minimize the challenges of GHEs, but the voices of host stakeholders should be equally heard.

\section{Social Justice Lens.}

The social justice lens I use to critically appraise my study findings is based on Nancy Fraser’s work. Fraser defines justice as "parity of participation”. (Fraser \& Olson, 2008) She explains that:

"According to this radical-democratic interpretation of the principle of equal moral worth, justice requires social arrangements that permit all to participate as peers in social life. Overcoming injustice means dismantling institutionalized obstacles that prevent some 
people from participating on a par with others as full partners in social interaction.” (Fraser \& Olson, 2008)

The lack of social justice within GHEs is most evident in the perspectives of local trainees. Although VMTs were perceived to come to LMIC to 'touch patients', local trainees perceived that they were not provided the same opportunity to interact with patients when going on a HIC exchange. This challenge is acknowledged in Pitt et al. 's (2016) description of a bilateral exchange program as their institutional regulations prevent LMIC trainees from hands-on engagement. Many VMTs may be present at a LMIC host site at one time, and each sending institution presumably expects their trainee to be afforded active and engaged learning opportunities. If I turn this around, and thirty LMIC trainees, with little to no knowledge of English, appeared at a HIC healthcare facility expecting engagement with patients, how would physicians feel in supervising and providing feedback to these LMIC trainees while also supervising their own trainees? Institutionalized obstacles need to be dismantled to allow LMIC trainees to participate in bilateral exchanges in the same way VMTs can participate at the LMIC host site. Furthermore, given that VMTs achieve significant benefit from a GHE but create challenges for the LMIC host site, a social justice perspective would demand that host stakeholders receive similar opportunities, and that financial and institutional barriers to bilateral GHEs must be overcome.

\section{Social Accountability Lens.}

I return to social accountability here, as discussed in Chapter 1, to describe how GHEs should be socially accountable and I caution HIC academic institutions regarding the need to continue to critically examine whether GHEs are truly socially accountable. I use the concept of social accountability of a medical school as the practice of engaging with and being held to 
account by the communities the medical school has a mandate to serve. (Boelen \& Woollard, 2011) To indeed be socially accountable, HIC academic institutions must be responsive to the concerns identified by host stakeholders in my study. If GHEs continue in their current forms, VMTs and HIC academic institutions will continue to benefit to the overall detriment of the host sites. Although host sites would continue to gain some benefits as acknowledged by participants in this study, most of the benefit would still be for the VMTs. Stating that host sites gain some benefits and using that as a justification to continue GHEs in their current formats, but ignoring the concerns identified by this study, would be a desecration of a medical school's mandate to be socially accountable. Therefore, a socially accountable perspective prompts the need for responsiveness based on the study findings.

\section{Implications for Practice.}

Finally, I could draw several conclusions from this study: to discontinue GHEs given the significant challenges, harms, and unintended consequences; to continue GHEs in their current forms as overall host stakeholders state they wish GHEs to continue; or to improve the process of GHEs through more deliberate, bidirectional, and mutually beneficial programs. I argue that if academic institutions discontinue GHEs this may significantly damage the relationships built with LMIC partner institutions by indicating a lack of trust in their ability to provide unique learning opportunities for VMTs. Although this may relieve some of the burdens of host institutions and communities regarding human and physical resource strain, this is a radical solution which may also have unintended harmful consequences. Secondly, although overall host stakeholders mentioned they wished for GHEs to continue, this is not an excuse for HIC academic institutions to continue GHEs in their current forms. As I identified, there are 
significant challenges and harms, and if these are not addressed, the purpose of GHEs in learning about global health inequities will be counteracted by the very experience itself. This deliberation leads me to consider the last conclusion as the only possible solution: The process of GHEs must be improved through more deliberate, bidirectional, and mutually beneficial programs. Utilizing the lenses described, I provide a list of recommendations from my study findings for sending and host institutions, and for VMTs (Table 5 and Table 6). Most importantly, these recommendations are exceedingly difficult to achieve if VMTs are allowed to select their own host site. Therefore, GHEs should occur within well-structured programs, developed and maintained between the HIC sending institution and the LMIC host institution. My recommendations aim to refine the approach and provide practical suggestions based on the guidelines set out by WEIGHT. Crump et al. (2010) in setting out the WEIGHT guidelines, encouraged work aimed at assessing the potential benefits and harms to institutions, personnel, trainees, patients and the community in host countries of global health training programs, to thereby refine the guidelines. Although at the time of their guideline development process the authors acknowledged there was limited evidence to inform their guidelines, the guidelines are comprehensive and provide a good framework for my recommendations. This framework includes guidelines for sending and host institutions, trainees, and sponsors of global health training programs. I did not include a separate table for sponsors of global health training programs because the guidelines for sponsors are covered within the guidelines for sending and host institutions. In the Canadian context, typically the sending institution is also the sponsor. I have condensed some of the WEIGHT guidelines in the following tables. 
Table 5 Recommendations and Practical Suggestions for Sending and Host Institutions

\begin{tabular}{|c|c|}
\hline \multicolumn{2}{|c|}{$\begin{array}{l}\text { WEIGHT Guidelines for Sending and Host } \\
\text { Institutions }\end{array}$} \\
\hline 1. & $\begin{array}{l}\text { Develop well-structured programs, so that } \\
\text { host and sender, as well as other stakeholders, } \\
\text { derive mutual, equitable benefit including: } \\
\text { a. Discuss expectations and } \\
\text { responsibilities of both host and } \\
\text { sending institutions and agree on } \\
\text { terms before program } \\
\text { implementation (consider an MOU), } \\
\text { and revisit these on a periodic basis } \\
\text { b. Consider local needs and priorities } \\
\text { regarding the optimal structure of the } \\
\text { program } \\
\text { c. Recognize the true cost to all } \\
\text { institutions and ensure that they are } \\
\text { appropriately reimbursed } \\
\text { d. Aspire to maintain long-term } \\
\text { relationships so that short-term } \\
\text { experiences may be nested within } \\
\text { them } \\
\text { e. Promote transparency regarding the } \\
\text { motivations for establishing and } \\
\text { maintaining programs, identify and } \\
\text { address any conflicts of interest and } \\
\text { conflicts of obligations that may } \\
\text { result from such a program. }\end{array}$ \\
\hline
\end{tabular}

Practical Suggestions based on my study findings

- $\quad$ Specify how GHEs fit within a formal institutional collaboration (ie. MOU and/or Supplementary Letter of Agreement) with agreed upon roles and responsibilities for sending and host institutions

- Discuss a strategy for the collaboration that promotes mutual benefit for local trainees within bilateral exchange opportunities

- Acknowledge and agree upon what costs/ contributions the sending and host institutions are contributing for GHEs within the collaboration

- Communicate regularly between sending and host institutions regarding successes and challenges of the GHE program

- Evaluate the GHE program within the collaboration at designated intervals that includes input from host preceptors / staff, local trainees and VMTs

- Envisage costs and benefits in the context of improving quality at host institutions

- Encourage host institutions to develop an International Relations Desk that processes and schedules GHE applications in consultation with host departments and collects local preceptor assessment and VMT evaluations

- Encourage host institutions to have a centralized system to track VMTs to tabulate numbers and their location

- Encourage host institutions to decline VMT applications if they have too many requests or insufficient capacity

- Follow international guidelines if research is being developed that involves VMT participation

2. Clarify goals, expectations, and responsibilities through explicit agreements and periodic review
- Develop mutual understanding of what global health competencies* are and which ones will be the areas of focus, preferably within a bilateral exchange GHE program

- Encourage dissemination to host preceptors and staff concerning the goals and purpose of GHES

- Place VMTs on teaching services based upon capacity of the service, availability of host preceptors, and presence of local learners, as 


\begin{tabular}{|c|c|}
\hline & 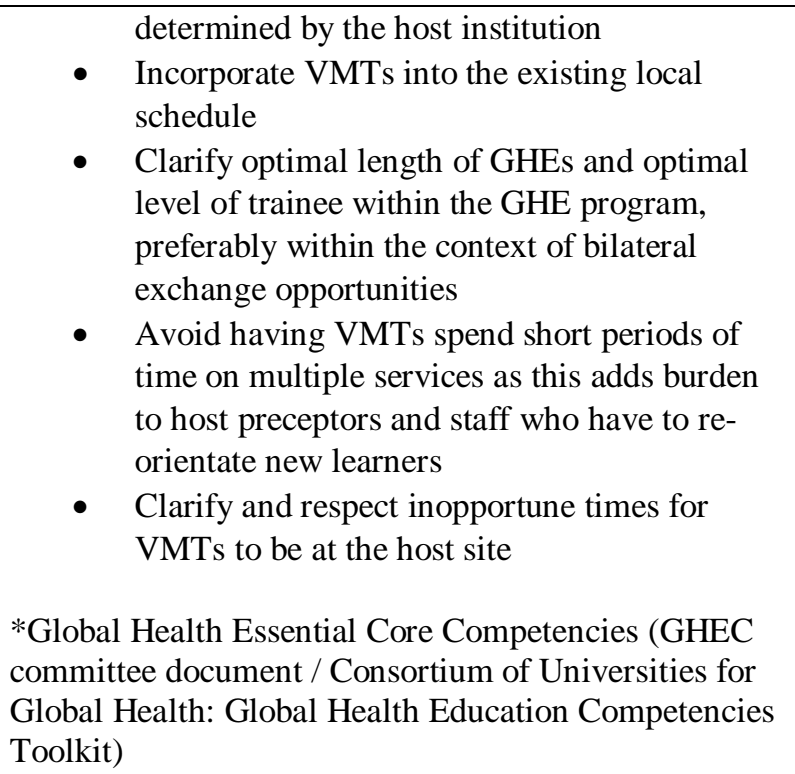 \\
\hline $\begin{array}{l}\text { 3. Develop, implement, regularly update, and } \\
\text { improve formal training for trainees and } \\
\text { mentors, both local and foreign (norms of } \\
\text { professionalism, standards of practice, cultural } \\
\text { competence, language, etc.) }\end{array}$ & $\begin{array}{l}\text { - Seek agreement upon what global health } \\
\text { competencies should serve as general learning } \\
\text { objectives that are appropriate for local } \\
\text { trainees and VMTs } \\
\text { Mandate pre-departure training to VMTs that } \\
\text { includes teaching and learning on } \\
\text { professionalism as a visitor to a foreign } \\
\text { setting, cultural competency, and emotional } \\
\text { self-regulation } \\
\text { - Develop code of conduct for VMTs that is } \\
\text { agreed upon by both institutions and signed by } \\
\text { all VMTs prior to departure } \\
\text { Discuss strategy that would help to enable } \\
\text { host preceptors to address VMT emotional } \\
\text { challenges } \\
\text { Develop specific medical knowledge and skill } \\
\text { learning objectives before the GHE that is } \\
\text { reviewed by the sending institution, VMT, } \\
\text { and host institution (as well as the host } \\
\text { preceptor, if possible) } \\
\text { Consider making logbooks mandatory so host } \\
\text { preceptors are aware of VMT learning } \\
\text { experiences especially as host preceptors can } \\
\text { change during the GHE } \\
\text { Discuss if sending a visiting preceptor within } \\
\text { the institutional collaboration would be } \\
\text { beneficial in providing clinical teaching to } \\
\text { local students or enhancing knowledge (ie. } \\
\text { medical education pedagogy) to host } \\
\text { preceptors }\end{array}$ \\
\hline $\begin{array}{l}\text { 4. Encourage non-threatening communication to } \\
\text { resolve ethical conflicts. }\end{array}$ & $\begin{array}{ll}\text { - } & \text { Assess VMT professional behavior by } \\
\text { including such attributes on the assessment }\end{array}$ \\
\hline
\end{tabular}




\begin{tabular}{|c|c|}
\hline & $\begin{array}{l}\text { form that host preceptors fill in } \\
\text { - Develop protocol and communication strategy } \\
\text { that can provide a safe place to discuss ethical } \\
\text { concerns arising from GHEs }\end{array}$ \\
\hline $\begin{array}{l}\text { 5. Clarify the trainees' level of training and } \\
\text { experience for the host institution }\end{array}$ & $\begin{array}{l}\text { - Resolve the issue of 'lack of distinctiveness' } \\
\text { in the VMT role and level of training by } \\
\text { developing clear identification badges for } \\
\text { VMTs that includes level of training in local } \\
\text { language }\end{array}$ \\
\hline $\begin{array}{l}\text { 6. Select trainees who are adaptable, sensitive, } \\
\text { willing to listen and learn. }\end{array}$ & 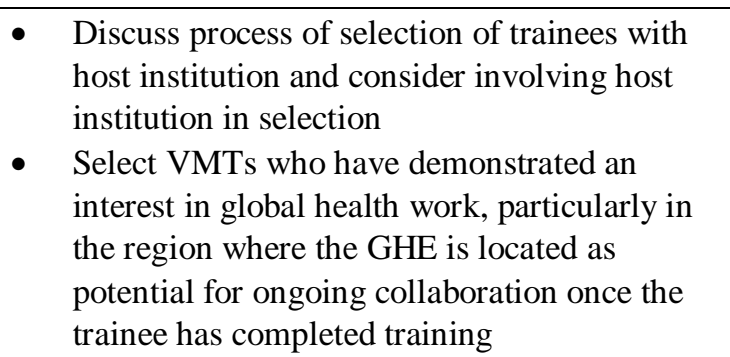 \\
\hline $\begin{array}{l}\text { 7. Promote safety of trainees to the extent } \\
\text { possible. }\end{array}$ & $\begin{array}{l}\text { - Mandate pre-departure training to VMTs that } \\
\text { includes teaching and learning about personal } \\
\text { health and travel safety }\end{array}$ \\
\hline $\begin{array}{l}\text { 8. Monitor costs and benefits to host institutions, } \\
\text { local trainees, patients, communities, and } \\
\text { sponsoring institutions to assure equity. }\end{array}$ & $\begin{array}{l}\text { - } \\
\text { Review the program on a regular basis } \\
\text { evaluating the costs and benefits to host } \\
\text { institution, local trainees, patients and } \\
\text { communities } \\
\text { Discuss with VMTs prior to departure issues } \\
\text { associated with bringing financial or physical } \\
\text { resources and if such donations are made, it } \\
\text { should be made transparent to the host } \\
\text { institution }\end{array}$ \\
\hline $\begin{array}{l}\text { 9. Establish effective supervision and } \\
\text { mentorship of trainees by the host and sending } \\
\text { institution. }\end{array}$ & 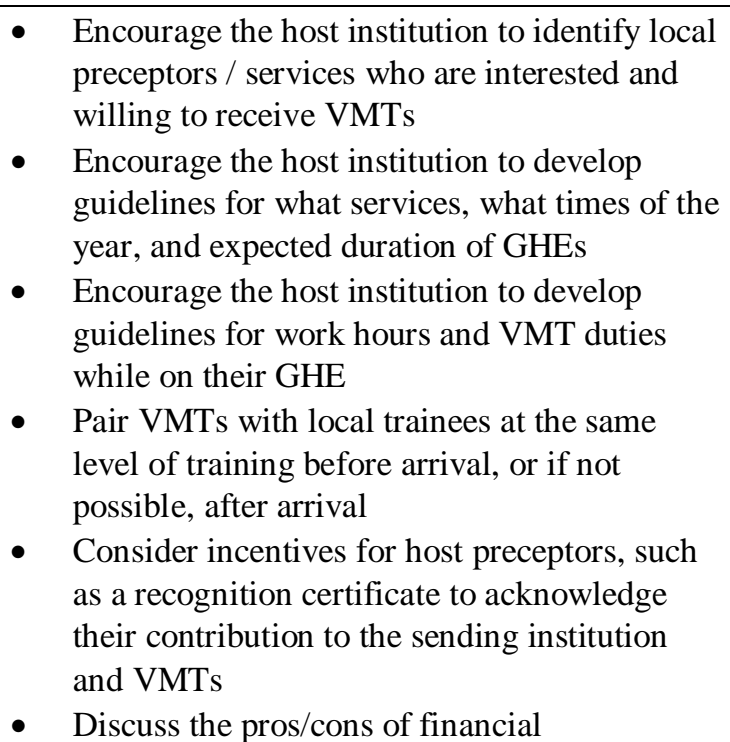 \\
\hline
\end{tabular}




\begin{tabular}{|c|c|}
\hline & $\begin{array}{l}\text { remuneration to host preceptors and develop } \\
\text { consensus on what seems appropriate within } \\
\text { the collaboration } \\
\text { Discuss the benefits and challenges of sending } \\
\text { a visiting preceptor within the institutional } \\
\text { collaboration and if appropriate, identify roles } \\
\text { and responsibilities of visiting preceptor }\end{array}$ \\
\hline $\begin{array}{l}\text { 10. Establish methods to solicit feedback from } \\
\text { trainees during and on completion of the GHE } \\
\text { and track participants post-training to evaluate } \\
\text { impact. }\end{array}$ & $\begin{array}{l}\text { - Mandate post-return debriefing with VMTs to } \\
\text { get feedback on elective } \\
\text { Develop standardized evaluation forms that } \\
\text { the VMT and VMT-assigned local trainee } \\
\text { complete at the end of the GHE } \\
\text { - } \\
\text { Review VMT and VMT-assigned local trainee } \\
\text { evaluations on a regular basis between } \\
\text { collaborating institutions summarizing VMT } \\
\text { and local trainee learning and experiences, } \\
\text { preferably within the context of bilateral } \\
\text { exchange opportunities } \\
\text { Provide host preceptors annual feedback } \\
\text { summary on the GHE experiences of local } \\
\text { learners and VMTs who were on their service }\end{array}$ \\
\hline
\end{tabular}


Table 6 Recommendations and Practical Suggestions for Visiting Medical Trainees

\begin{tabular}{|c|c|}
\hline WEIGHT guidelines for VMTs & $\begin{array}{l}\text { Practical Suggestions based on my study } \\
\text { findings }\end{array}$ \\
\hline $\begin{array}{l}\text { 1. Recognize that the primary purpose of the } \\
\text { experience is global health learning and } \\
\text { appropriately supervised service. Duration of } \\
\text { the training experience should be tailored so } \\
\text { burden to the host is minimized. }\end{array}$ & $\begin{array}{l}\text { Discuss with host preceptor the global health } \\
\text { competencies you hope to focus on during the } \\
\text { GHE } \\
\text { Discuss your specific medical knowledge and } \\
\text { skill learning objectives with your host } \\
\text { preceptor and review when new host } \\
\text { preceptor begins to supervise the service } \\
\text { - Seek to join a supervised service and become } \\
\text { an active member of the team } \\
\text { - Recognize that hosts appreciate active } \\
\text { participation of VMTs in patient care, but } \\
\text { balance this with appropriate supervision } \\
\text { Seek to follow schedules of local trainees, } \\
\text { including participation in overnight and } \\
\text { weekend call } \\
\text { Avoid sampling multiple specialties at the } \\
\text { host site for short periods of time (touristic } \\
\text { behaviour), rather seek to build rapport within } \\
\text { one team / ward / specialty } \\
\text { Plan tourist activities outside of the elective } \\
\text { block so that you can remain an active } \\
\text { member of the team, including taking call } \\
\text { with local trainees }\end{array}$ \\
\hline $\begin{array}{l}\text { 2. Communicate with local mentor through } \\
\text { official channels regarding goals and } \\
\text { expectations before the training. }\end{array}$ & $\begin{array}{l}\text { - } \begin{array}{l}\text { Ensure your host preceptor knows your level } \\
\text { of training }\end{array} \\
\text { - Review learning objectives with the host } \\
\text { preceptor whenever there is a change of } \\
\text { preceptor on the service } \\
\text { - Introduce yourself to nurses and local trainees } \\
\text { on the service and explain your learning } \\
\text { objectives }\end{array}$ \\
\hline $\begin{array}{l}\text { 3. Learn appropriate language skills relevant to } \\
\text { the host's locale as well as socio-cultural, } \\
\text { political, and historical aspects. }\end{array}$ & 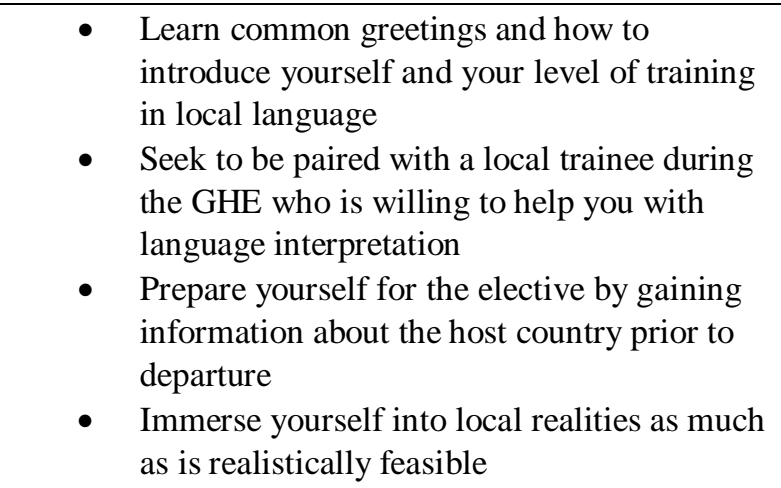 \\
\hline
\end{tabular}




\begin{tabular}{|c|c|c|}
\hline & $\begin{array}{l}\text { Seek to acquire knowledge and learn new } \\
\text { skills with appropriate training and } \\
\text { supervision but be cognizant and respectful of } \\
\text { their current capability and level of training. }\end{array}$ & $\begin{array}{l}\text { Consult with host preceptors about potential } \\
\text { role in teaching more junior local trainees } \\
\text { recognizing you are not an expert on local } \\
\text { diagnosis and treatment strategies / Consider } \\
\text { teaching research skills, sharing free online } \\
\text { educational resources, conducting literature } \\
\text { searches with local trainees, leading a journal } \\
\text { club on a locally relevant topic, helping to } \\
\text { write a journal article } \\
\text { Respect the decisions and requests of local } \\
\text { staff, including your preceptor, ward nurses, } \\
\text { and clinical officers and avoid trying to look } \\
\text { superior in knowledge }\end{array}$ \\
\hline 5. & $\begin{array}{l}\text { Participate in the process of communicating to } \\
\text { patients and the community about their level } \\
\text { of training and experience. }\end{array}$ & $\begin{array}{l}\text { - Wear clearly visible identification that } \\
\text { includes your level of training written in local } \\
\text { language } \\
\text { - Learn some local language to enable you to } \\
\text { ask patients a few questions for history-taking }\end{array}$ \\
\hline 6. & $\begin{array}{l}\text { Recognize and respect divergent diagnostic } \\
\text { and treatment paradigms. }\end{array}$ & $\begin{array}{l}\text { - Seek to understand local diagnostic and } \\
\text { treatment paradigms and avoid making } \\
\text { judgmental comparisons to diagnostic and } \\
\text { treatment guidelines from your home } \\
\text { institution }\end{array}$ \\
\hline 7. & $\begin{array}{l}\text { Demonstrate cultural competency and engage } \\
\text { in appropriate discussions about different } \\
\text { perspectives and approaches. }\end{array}$ & $\begin{array}{l}\text { - } \quad \text { Avoid interacting predominantly with other } \\
\text { VMTs and seek to socialize with local trainees } \\
\text { - } \quad \text { Seek to learn about local customs } \\
\text { - }\end{array}$ \\
\hline 8. & $\begin{array}{l}\text { Take measures to ensure personal safety and } \\
\text { health. }\end{array}$ & $\begin{array}{l}\text { - Seek and respect the security advice given by } \\
\text { hosts } \\
\text { - Communicate with hosts about destinations if } \\
\text { leaving for a weekend } \\
\text { - Attend pre-departure training at your sending } \\
\text { institution } \\
\text { - Gain knowledge of common local } \\
\text { conditions/outbreaks so as to take appropriate } \\
\text { precautions }\end{array}$ \\
\hline 9. & $\begin{array}{l}\text { Meet licensing standards, visa policies, } \\
\text { research ethics review, training on privacy and } \\
\text { security of patient information, and other host } \\
\text { and sending country requirements. }\end{array}$ & $\begin{array}{l}\text { - } \begin{array}{l}\text { Get license for host country prior to elective } \\
\text { (as required by host country) }\end{array} \\
\text { - } \quad \text { Ask host preceptor about guidelines around } \\
\text { photography } \\
\text { - Maintain the same standards as your sending } \\
\text { institution concerning patient confidentiality } \\
\text { - Be cautious about what you post on blogs or } \\
\text { electronic media }\end{array}$ \\
\hline 10. & $\begin{array}{l}\text { Follow accepted international guidelines } \\
\text { regarding the donation of medications, } \\
\text { technology, and supplies. }\end{array}$ & $\begin{array}{l}\text { - } \quad \text { Be cognizant of potential implications in } \\
\text { handing out materials or making financial }\end{array}$ \\
\hline
\end{tabular}




\begin{tabular}{|c|c|}
\hline & $\begin{array}{l}\text { donations randomly to patients as this may } \\
\text { create unrealistic expectations } \\
\text { - Follow guidelines of your sending institution } \\
\text { with respect to material and financial } \\
\text { donations } \\
\text { - If donations are planned, inform the host } \\
\text { institution of the donation and seek their input }\end{array}$ \\
\hline $\begin{array}{l}\text { 11. If research is part of the training experience, } \\
\text { develop the research plan early, in } \\
\text { consultation with mentors, focus on research } \\
\text { themes of interest and relevance to the host, } \\
\text { understand and follow all research procedures } \\
\text { of the host and sending institution, obtain } \\
\text { ethics approval }\end{array}$ & 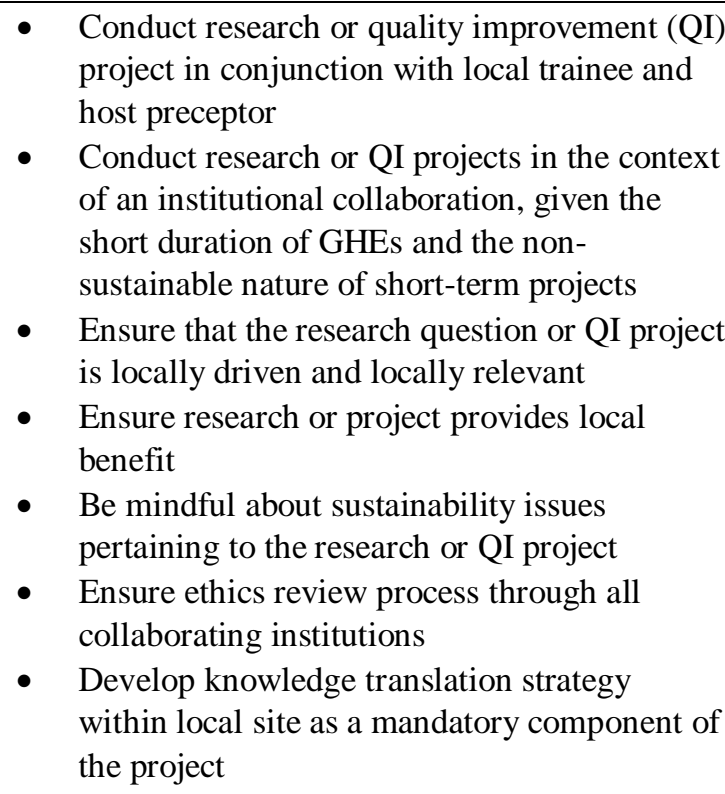 \\
\hline $\begin{array}{l}\text { 12. Follow international standards for authorship } \\
\text { of publications emanating from the global } \\
\text { health experiences }\end{array}$ & $\begin{array}{l}\text { - Include local involvement and authorship on } \\
\text { all research or QI project publications } \\
\text { conducted at a host institution } \\
\text { - Acknowledge your local research participants }\end{array}$ \\
\hline $\begin{array}{l}\text { 13. Be willing to share feedback on the training } \\
\text { experience and follow-up information on } \\
\text { career progression }\end{array}$ & 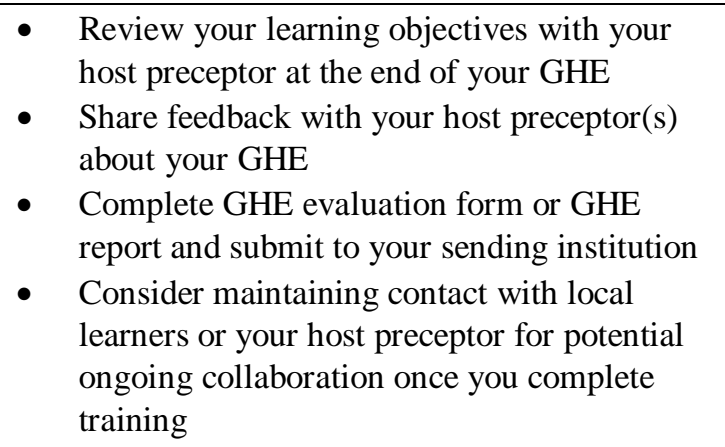 \\
\hline $\begin{array}{l}\text { 14. When seeking global health training outside } \\
\text { of a well-structured program, potential } \\
\text { trainees should follow the guidelines for } \\
\text { institutions (see Table } 1 \text { ) so as to maximize } \\
\text { the benefits and minimize potential harms of } \\
\text { such training experiences. }\end{array}$ & $\begin{array}{l}\text { - Choose NOT to do a GHE outside of an } \\
\text { institutional collaboration program, especially } \\
\text { for medical students and junior residents } \\
\text { - Consider GHE as a senior resident outside of } \\
\text { an institutional collaboration program, but } \\
\text { ideally in the context of ongoing partnership } \\
\text { with the host institution upon completion of } \\
\text { training }\end{array}$ \\
\hline
\end{tabular}




\section{Implications for research.}

This study revealed that global health electives among host stakeholders in Tanzania and Uganda, as reported in the participants’ accounts, is a commonly-experienced phenomenon, providing some benefit. However, these GHEs still present significant challenges, burdens, and unintended consequences which must be addressed. Although the study findings provide further evidence for the ethics and best practice guidelines developed by the WEIGHT group and new understanding on some host stakeholder perspectives not previously reported, there are yet certain aspects within this phenomenon which need to be further elucidated. Three areas of future research include the benefits and disadvantages of foreign preceptors accompanying VMTs, the impact of research or quality improvement projects by VMTs during a clinical GHE, and whether exchange opportunities for local trainees improve mutual benefit. My study findings were equivocal as to whether host sites preferred foreign preceptors to accompany VMTs. I suggest further qualitative studies to explore the benefits and disadvantages of foreign preceptors accompanying VMTs from the perspectives of host stakeholders (staff and trainees), foreign preceptors, and VMTs. Secondly, although small research or quality improvement projects were viewed as beneficial at the rural host site, overall research projects by VMTs at the two urban host sites were not viewed as beneficial. Further exploration of what constitutes a successful research project conducted by a VMT should be studied and whether these research projects truly provide a positive impact to host communities. Lastly, based on my study findings I recommend each sending institution provide bilateral exchange opportunities. Given that bilateral exchange programs for LMIC trainees are infrequently described in the literature, further research should be conducted to evaluate the impact of bilateral exchange programs. (Abedini et al., 2014; Pitt et al., 2016; Umoren et al., 2014) In conclusion, although my study 
adds significant knowledge to the field regarding global health electives from host stakeholder perspectives, the stark imbalance of host versus visitor perspectives included in primary studies on global health electives should continue to motivate further exploration and understanding of host stakeholder perspectives.

\section{Limitations of the study}

Although I achieved the aims of the study objectives, there were some limitations that may impact the study findings. The first limitation relates to language barrier. The majority of interviews in Tanzania and two interviews in Uganda were conducted in the local language. Despite careful review of the translations, some portions of interviews were still difficult to understand and interpret. Similarly, with the translated interview guides participants may not have completely understood or misinterpreted the questions. A second limitation is that although both interview moderators had previous experience conducting semi-structured interviews and focus groups, they still required significant coaching regarding probing and in certain transcripts I identified some leading questions. This may affect the themes within my data. Another limitation is that the final step in data analysis for qualitative research, member-checking, was not done. Member checking involves taking data and interpretations back to the participants in the study and determining whether these participants feel the analysis is credible. (Creswell, 2013) Due to the logistical considerations of this study, these steps were not feasible as I had already returned to

Canada before reaching this step in the process. The findings, as a result, may not adequately reflect the participants' experience thereby impacting the credibility of findings. However, four other individuals separately analyzed the interviews and focus groups, which provided a measure 
of trustworthiness. Also, I was able to meet in person with two of the local research collaborators from Tanzania and Uganda in June 2018 to discuss the research findings and interpretations. Furthermore, courtesy bias is a limitation that may be present in the results given that participants may not have wanted to jeopardize established partnerships. As discussed, I attempted to mitigate this by not being present during the interviews and focus groups. During the analysis phase, in discussion with my committee, we agreed that there was sufficient critique provided within most of the interviews that courtesy bias was likely not a significant concern. One quote provided by a local trainee provides evidence that courtesy bias was mitigated by myself not being present as it is unlikely this would have been discussed had I been present (as a Caucasian female foreigner):

"At the end of the day [...] whenever you come to the ward instead of focusing on the, on the cases, focusing on the knowledge, for you, your mind will be thinking [...] how will I be able to, to win this female foreign student into a relationship.”

Furthermore, I could have included more host sites or interviewed more participants for my study to mitigate these limitations and improve the credibility and transferability of my study findings. However, as previously discussed, this was not possible due to practical reasons (i.e., time, budget). Although the findings of my study are context-specific, I argue that there is significant transferability to other sites. I provide a detailed description of the settings which allows other institutions to determine what is applicable to their site. The study findings are also reflective of studies previously conducted on host stakeholder perspectives in the Caribbean, South America, India, and other countries within Africa suggesting that host stakeholders' experience of global health electives is somewhat similar across LMIC host sites. I argue that interviewing more participants would likely not have yielded new findings. The majority of themes that emerged from the interviews and focus groups in Tanzania were repeated in Uganda. 
Despite the limitations, I was able to achieve my study objectives to: 1) Explore and explain perspectives regarding the harms, benefits, and other impacts of global health electives from multiple stakeholders; and 2) Explore and explain perspectives on how to create more equitable and mutually beneficial global health electives from multiple stakeholders.

\section{Conclusion}

I explored the phenomenon of global health electives through the accounts of health facility administrators, physicians, clinical officers, registrars, nurses, local trainees, patients, one patient' family member, and community liaison members living in Mwanza, Tanzania, Rugazi, Uganda and Mbarara, Uganda. Although there were nuances within each of the themes and several differences in perspectives were found between Uganda and Tanzania, urban and rural sites, and stakeholder roles, overall the majority of host stakeholders experienced the essence of the phenomenon in similar ways. Through the descriptions of participant' experiences, global health electives involved frequent experiences with VMTs from many different countries; however, often participants were unable to distinguish between the role of VMTs and other visitors. Participant perspectives on why VMTs come for a GHE somewhat differed from the perspectives of HIC academic institutions in that they perceived VMTs coming to 'touch patients' because they are unable to do this at their home institution or that it is a requirement to learn 'global health' through a global health elective. Several explicit and implicit reasons were provided as to why host stakeholders accept VMTs to come to the host site: they wish to portray themselves as welcoming, hosts' moral obligation to teach all trainees, lack of capacity to negotiate how many VMTs they supervise, and benefits for themselves as hosts and the host institution. However, although host stakeholders overall try to provide a positive overview of 
GHEs, as also supported by existing literature, my study found that there are significant harms, burdens, and unintended consequences of GHEs, some of which have not previously been reported. I contribute to global health and medical education literature new evidence that VMTs take away learning opportunities from local trainees and VMTs are perceived as more knowledgeable than local staff by patients. Despite these harms, burdens and unintended consequences, host stakeholders wish for GHEs to continue with the hope that HIC and LMIC institutions and health care facilities will collaborate to improve the process of GHEs and ensure mutual benefit for all stakeholders through the recommendations they provided. My hope is that this study has made it possible for participant voices to be heard and that recommendations from the study will be considered so that global health electives can be improved for all stakeholders involved. My study findings support many of the WEIGHT guidelines for ethics and best practice guidelines for training experiences in global health. I provided practical suggestions for the guidelines based on my study findings. Through the findings, dissemination, and suggestions for future work, this case-based interpretive phenomenological study will hopefully be the impetus for HIC and LMIC institutions to work together to create effective, ethical, and equitable electives within a structured collaboration and with a bidirectional focus. 


\section{References}

Abedini, N. C., Danso-Bamfo, S., Moyer, C. A., Danso, K. A., Makiharju, H., Donkor, P., .. . Kolars, J. C. (2014). Perceptions of Ghanaian medical students completing a clinical elective at the University of Michigan Medical School. Academic Medicine, 89(7), 10141017. doi:10.1097/ACM.0000000000000291

Abedini, N. C., Gruppen, L. D., Kolars, J. C., \& Kumagai, A. K. (2012). Understanding the effects of short-term international service-learning trips on medical students. Academic Medicine, 87(6), 820-828.

Ackerman, L. K. (2010). The Ethics of Short-Term International Health Electives in Developing Countries. Annals of Behavioral Science and Medical Education, 16(2), 40-43. doi:10.1007/bf03355131

Anderson, K. C., Slatnik, M. A., Pereira, I., Cheung, E., Xu, K., \& Brewer, T. F. (2012). Are we there yet? Preparing Canadian medical students for global health electives. Academic Medicine, 87. doi:10.1097/ACM.0b013e31823e23d4

Beaglehole, R., \& Bonita, R. (2010). What is global health? Global Health Action, 3, 10.3402/gha.v3403i3400.5142. doi:10.3402/gha.v3i0.5142

Boelen, C., \& Woollard, R. (2011). Social accountability: The extra leap to excellence for educational institutions. Medical Teacher, 33(8), 614-619. doi:10.3109/0142159X.2011.590248

Bozinoff, N., Dorman, K. P., Kerr, D., Roebbelen, E., Rogers, E., Hunter, A., . . Kraeker, C. (2014). Toward reciprocity: host supervisor perspectives on international medical electives. Medical Education, 48(4), 397-404.

Brada, B. (2011). "Not Here”: Making the Spaces and Subjects of "Global Health” in Botswana. Culture, Medicine, and Psychiatry, 35(2), 285-312. doi:10.1007/s11013-011-9209-z

Braun, V., \& Clarke, V. (2013). Stages of coding and analysis described by different qualitative analytic methods. . In Successful Qualitative Research: A Practical Guide for Beginners. London: Sage.

Canadian Institutes of Health Research, Natural Sciences and Engineering Research Council of Canada, \& Social Sciences and Humanities Research Council of Canada. (2010). Tricouncil policy statement: Ethical conduct for research involving humans (TCPS 2). Retrieved from http://www.pre.ethics.gc.ca/pdf/eng/tcps22010/TCPS_2_FINAL_Web.pdf

Catholic University of Health and Allied Sciences. (2018). Overview of CUHAS. Retrieved from http://www.bugando.ac.tz/about.php\#history

Commonwealth, S. (2018a). Uganda : History. Retrieved from http://thecommonwealth.org/ourmember-countries/uganda/history

Commonwealth, S. (2018b). United Republic of Tanzania : History. Retrieved from http://thecommonwealth.org/our-member-countries/united-republic-tanzania/history

Crane, J. T. (2013). Scrambling for Africa : AIDS, Expertise, and the Rise of American Global Health Science. Ithaca, United States: Cornell University Press.

Creswell, J. W. (2013). Qualitative Inquiry\&Research Design: Choosing Among Five Approaches (3 ed.). California: Sage.

Crump, J. A., \& Sugarman, J. (2008). Ethical considerations for short-term experiences by trainees in global health. JAMA, 300. doi:10.1001/jama.300.12.1456

Crump, J. A., Sugarman, J., \& the Working Group on Ethics Guidelines for Global Health 
Training. (2010). Ethics and Best Practice Guidelines for Training Experiences in Global Health. The American Journal of Tropical Medicine and Hygiene, 83(6), 1178-1182. doi:https://doi.org/10.4269/ajtmh.2010.10-0527

DeCamp, M., Enumah, S., O'Neill, D., \& Sugarman, J. (2014). Perceptions of a short-term medical programme in the Dominican Republic: Voices of care recipients. Global Public Health, 9(4), 411-425. doi:10.1080/17441692.2014.893368

Dell, E. M., Varpio, L., Petrosoniak, A., Gajaria, A., \& McCarthy, A. E. (2014). The ethics and safety of medical student global health electives. International Journal of Medical Education, 5, 63-72. doi:10.5116/ijme.5334.8051

Denzin, N. K., \& Lincoln, Y. S. (1994). Handbook of qualitative research. Thousand Oaks, California: Sage.

Drain, P. K., Holmes, K. K., Skeff, K. M., Hall, T. L., \& Gardner, P. (2009). Global health training and international clinical rotations during residency: current status, needs, and opportunities. Academic Medicine, 84. doi:10.1097/ACM.0b013e3181970a37

Dwyer, J. (2003). Teaching Global Bioethics. Bioethics, 17(5-6), 432-446. doi:10.1111/14678519.00359

Dwyer, J. (2005). Global Health and Justice. Bioethics, 19(5/6), 460-475. doi:10.1111/j.14678519.2005.00458.x

Easterly, W. (2007). The White Man's Burden: Why the West's Efforts to Aid the Rest Have Done So Much Ill and So Little Good. London, England.: Penguin Books.

Edwards, R., Piachaud, J., Rowson, M., \& Miranda, J. (2004). Understanding global health issues: are international medical electives the answer? Medical Education, 38(7), 688690. doi:doi:10.1111/j.1365-2929.2004.01849.x

Elit, L., Hunt, M., Redwood-Campbell, L., Ranford, J., Adelson, N., \& Schwartz, L. (2011). Ethical issues encountered by medical students during international health electives. Medical Education, 45. doi:10.1111/j.1365-2923.2011.03936.x

Elobu, A. E., Kintu, A., Galukande, M., Kaggwa, S., Mijjumbi, C., Tindimwebwa, J., .. . Lipnick, M. (2014). Evaluating international global health collaborations: perspectives from surgery and anesthesia trainees in Uganda. Surgery, 155(4), 585-592. doi:https://dx.doi.org/10.1016/j.surg.2013.11.007

Evans, T., Akporuno, O., Owens, K. M., Lickers, B., Marlinga, J., Lin, H. C., \& Loh, L. C. (2017). Using Maslow's Hierarchy to Highlight Power Imbalances between Visiting Health Professional Student Volunteers and the Host Community: An Applied Qualitative Study. Education for Health: Change in Learning \& Practice (Medknow Publications \& Media Pvt. Ltd.), 30(2), 133-139. doi:10.4103/efh.EfH_175_16

Evert, J. S., C. Chan, K. Rosenberg, M. Hall, T. (2008). Developing Residency Training in Global Health: A Guidebook. San Francisco.

Fraser, N., \& Olson, K. (2008). Adding insult to injury: Nancy fraser debates her critics. London; New York: Verso.

Frischtak, H., \& Sinha, P. (2013). The hitchhiker's guide to global health blogging. Perspectives in Biology and Medicine, 56(4), 602-610. doi:10.1353/pbm.2013.0039

Glaser, B. G., \& Strauss, A. L. (1967). The discovery of grounded theory: Strategies for qualitative research. Chicago: Aldine.

Global Consensus for Social Accountability of Medical Schools. (2010). San Francisco.

Godkin, M., \& Savageau, J. (2003). The effect of medical students' international experiences on attitudes toward serving underserved multicultural populations. Family Medicine, 35. 
Government of Canada. (2017). Canadian international assistance in Tanzania. Retrieved from http://www.international.gc.ca/world-monde/issues_developmentenjeux developpement/priorities-priorites/where-ou/tanzania-tanzanie.aspx?lang=eng

Green, J., \& Thorogood, N. (2014). Qualitative Methods for Health Research (3 ed.). London: Sage.

Guba, E. E., \& Lincoln, Y. S. (1981). Effective Evaluation. San Francisco: Jossey-Bass.

Hanson, L., Harms, S., \& Plamondon, K. (2011). Undergraduate International Medical Electives: Some Ethical and Pedagogical Considerations. Journal of Studies in International Education, 15(2), 171-185. doi:doi:10.1177/1028315310365542

Hayward, L. M., \& Li, L. (2017). Sustaining and improving an international service-learning partnership: Evaluation of an evidence-based service delivery model. Physiotherapy Theory \& Practice, 33(6), 475-489. doi:10.1080/09593985.2017.1318425

Hebert, P. C. (2008). Doing Right: a practical guide to ethics for medical trainees and physicians (2nd ed ed.). New York: Oxford University Press.

Holmes, D., Zayas, L., \& Koyfman, A. (2012). Student Objectives and Learning Experiences in a Global Health Elective. Journal of Community Health, 37(5), 927-934. doi:10.1007/s10900-012-9547-y

Iwowo, V. (2014). Post-Colonial Theory. In D. Coghlan, Brydon-Miller, M. (Ed.), The SAGE Encyclopedia of Action Research. London.

Kickbusch, I. (2006). The need for a European Strategy on Global Health. Scandinavian Journal of Social Medicine, 34(6), 561-565. doi:10.1080/14034940600973059

Koch, T. (1994). Establishing rigour in qualitative research: the decision trail. Journal of Advanced Nursing, 19(5), 976-986. doi:10.1111/j.1365-2648.1994.tb01177.x

Koch, T. (1996). Implementation of a hermeneutic inquiry in nursing: philosophy, rigour and representation. Journal of Advanced Nursing, 24(1), 174-184. doi:doi:10.1046/j.13652648.1996.17224.x

Koplan, J. P., Bond, T. C., Merson, M. H., Reddy, K. S., Rodriguez, M. H., \& Sewankambo, N. K. (2009). Towards a common definition of global health. Lancet, 373. doi:10.1016/s0140-6736(09)60332-9

Kumwenda, B., Dowell, J., Daniels, K., \& Merrylees, N. (2015). Medical electives in subSaharan Africa: a host perspective. Medical Education, 49(6), 623-633. doi:10.1111/medu.12727

Kung, T. H., Richardson, E. T., Mabud, T. S., Heaney, C. A., Jones, E., \& Evert, J. (2016). Host community perspectives on trainees participating in short-term experiences in global health. Medical Education, 50(11), 1122-1130.

Liaison Committee on Medical Education (LCME). (2017). Data Collection Instrument for Full Accreditation Surveys 2017-18 (11.3 Oversight of Extramural Electives). Retrieved from http://www.lcme.org/publications/

Lincoln, Y. S., \& Guba, E. E. (1985). Naturalistic Inquiry. Beverly Hills, CA: SAGE Publications.

Lincoln, Y. S., \& Guba, E. E. (1986). Research, Evaluation, and Policy Analysis: Heuristics for Disciplined Inquiry. Policy Studies Review, 5(3), 546-565.

Liu, Y., Zhang, Y., Liu, Z., \& Wang, J. (2015). Gaps in studies of global health education: an empirical literature review. Glob Health Action, 8, 25709.

Lukolyo, H., Rees, C. A., Keating, E. M., Swamy, P., Schutze, G. E., Marton, S., \& Turner, T. L. (2016). Perceptions and Expectations of Host Country Preceptors of Short-Term Learners 
at Four Clinical Sites in Sub-Saharan Africa. Academic Pediatrics, 16(4), 387-393.

Macfarlane, S. B., Jacobs, M., \& Kaaya, E. E. (2008). In the name of global health: trends in academic institutions. Journal of Public Health Policy, 29(4), 383-401.

Maley, M., Worley, P., \& Dent, J. (2009). Using rural and remote settings in the undergraduate medical curriculum: AMEE Guide No. 47. Medical Teacher, 31(11), 969-983. doi:10.3109/01421590903111234

Mbarara University of Science and Technology. (2018). Our History. Retrieved from http://www.must.ac.ug/about-must/must-profile/our-history

Ministry of Health. (2018). Mbarara Regional Referral Hospital Retrieved from http://www.health.go.ug/content/mbarara-regional-referral-hospital

Moskowitz, D., Glasco, J., Johnson, B., \& Wang, G. (2006). Students in the community: An interprofessional student-run free clinic. Journal of Interprofessional Care, 20(3), 254259. doi:10.1080/13561820600721091

Mutchnick, I. S., Moyer, C. A., \& Stern, D. T. (2003). Expanding the boundaries of medical education: evidence for cross-cultural exchanges. Academic Medicine, 78(10 Suppl), S15.

Overseas Development Institute. (2016). Case Study: Mwanza. Retrieved from https://www.odi.org/sites/odi.org.uk/files/resource-documents/11222.pdf

Oxford English Dictionary Online. Dynamics. Oxford English Dictionary Online.

Phillippi, J., \& Lauderdale, J. (2017). A Guide to Field Notes for Qualitative Research: Context and Conversation. Qualitative Health Research, 28(3), 381-388. doi:10.1177/1049732317697102

Pinto, A. D., \& Upshur, R. E. G. (2009). Global Health Ethics for Students. Developing World Bioethics, 9(1), 1-10. doi:10.1111/j.1471-8847.2007.00209.x

Pitt, M. B., Gladding, S. P., Majinge, C. R., \& Butteris, S. M. (2016). Making Global Health Rotations a Two-Way Street: A Model for Hosting International Residents. Global Pediatric Health, 3, 2333794X16630671. doi:10.1177/2333794X16630671

Purkey, E., \& Hollaar, G. (2016). Developing consensus for postgraduate global health electives: definitions, pre-departure training and post-return debriefing. BMC Medical Education, 16(1), 159. doi:10.1186/s12909-016-0675-4

Republic of Uganda. (2018). Facts and figures: Mbarara District. Retrieved from http://www.mbarara.go.ug/district/facts-and-figures

Ritz, S. A., Beatty, K., \& Ellaway, R. H. (2014). Accounting for social accountability: developing critiques of social accountability within medical education. Education for Health, 27(2), 152-157.

Sargeant, J. (2012). Qualitative Research Part II: Participants, Analysis, and Quality Assurance. Journal of Graduate Medical Education, 4(1), 1-3. doi:10.4300/JGME-D-11-00307.1

Shah, S., \& Wu, T. (2008a). The medical student global health experience: professionalism and ethical implications. Journal of Medical Ethics, 34(5), 375-378.

Shah, S., \& Wu, T. (2008b). The medical student global health experience: professionalism and ethical implications. Journal of Medical Ethics, 34. doi:10.1136/jme.2006.019265

Shenton, A. K. (2004). Strategies for ensuring trustworthiness in qualitative research projects. Education for Information, 22(2), 63-75.

Sheppes, G., Scheibe, S., Suri, G., \& Gross, J. J. (2011). Emotion-Regulation Choice. Psychological Science, 22(11), 1391-1396.

Simonelis, J., Njelesani, J., Novak, L., Kuzma, C., \& Cameron, D. (2011). International 
fieldwork placements and occupational therapy: Lived experiences of the major stakeholders. Australian Occupational Therapy Journal, 58(5), 370-377.

doi:10.1111/j.1440-1630.2011.00942.x

Simons, G. F., \& Fennig, C. D. (2018). Ethnologue: Languages of the World. 21st. Retrieved from https://www.ethnologue.com/country/UG

Smit, E. M., \& Tremethick, M. J. (2014). Preceptorship in an international setting: Honduran nurses and American nursing working together. Nurse Educator, 39(2), 91-95.

Smith, J. A. (2004). Reflecting on the development of interpretative phenomenological analysis and its contribution to qualitative research in psychology. Qualitative Research in Psychology, 1(1), 39-54. doi:10.1191/1478088704qp004oa

Smith, J. A., \& Osborn, O. (2003). Interpretive Phenomenological Analysis. In J. A. Smith (Ed.), Qualitative Psychology: a practical guide to research methods (2nd ed.). London: Sage.

Speziale, H. J., \& Carpenter, D. R. (2007). Qualitative Research in Nursing: Advancing the Humanistic Imperative (4th ed.). Philadelphia: Lippincott Williams \& Wilkins.

Starks, H., \& Brown Trinidad, S. (2007). Choose Your Method: A Comparison of Phenomenology, Discourse Analysis, and Grounded Theory. Qualitative Health Research, 17(10), 1372-1380. doi:10.1177/1049732307307031

Sullivan, N. (2018). International clinical volunteering in Tanzania: A postcolonial analysis of a Global Health business. Global Public Health, 13(3), 310-324. doi:10.1080/17441692.2017.1346695

Sykes, K. J. (2014). Short-Term Medical Service Trips: A Systematic Review of the Evidence. American Journal of Public Health, 104(7), e38-48.

The Health Infrastructure Working Group. (2011). Guidelines for Designation, Establishment, and Upgrading of Health Units.

The World Bank. World Bank Country and Lending Groups. Retrieved from https://datahelpdesk.worldbank.org/knowledgebase/articles/906519-world-bank-countryand-lending-groups

The World Bank. (2017). Tanzania | Data. Retrieved from https://data.worldbank.org/country/tanzania

Thompson, M. J., Huntington, M. K., Hunt, D. D., Pinsky, L. E., \& Brodie, J. J. (2003a). Educational effects of international health electives on U.S. and Canadian medical students and residents: a literature review. Academic Medicine, 78. doi:10.1097/00001888-200303000-00023

Thompson, M. J., Huntington, M. K., Hunt, D. D., Pinsky, L. E., \& Brodie, J. J. (2003b). Educational Effects of International Health Electives on U.S. and Canadian Medical Students and Residents: A Literature Review. Academic Medicine, 78(3), 342-347.

Umoren, R. A., Einterz, R. M., Litzelman, D. K., Pettigrew, R. K., Ayaya, S. O., \& Liechty, E. A. (2014). Fostering Reciprocity in Global Health Partnerships Through a Structured, Hands-On Experience for Visiting Postgraduate Medical Trainees. Journal of Graduate Medical Education, 6(2), 320-325. doi:10.4300/JGME-D-13-00247.1

Underwood, M., Gleeson, J., Konnert, C., Wong, K., \& Valerio, B. (2016). Global Host Partner Perspectives: Utilizing a Conceptual Model to Strengthen Collaboration with Host Partners for International Nursing Student Placements. Public Health Nursing, 33(4), 351-359. doi:10.1111/phn.12258

United Nations Development Programme. (2018). Human Development Reports. Retrieved from http://hdr.undp.org/en/countries 
University of Calgary. (2013). Becoming a Global Intellectual Hub: Highlights of the University of Calgary International Strategy. Retrieved from http://www.ucalgary.ca/research/files/research/becoming-a-global-intellectual-hub.pdf

University of Calgary. (2016). Enrich the Quality and Breadth of Learning: a focus group guide. Retrieved from https://www.ucalgary.ca/sites/default/files/documents/eyeshigh/Focus\%20Group\%20Guide\%20-\%20Teaching\%20-\%20061016.pdf

University of Calgary. (2018). Global Health Electives. Retrieved from http://www.ucalgary.ca/ghealth/education/electives

Waddell, W. H., Kelley, P.R., Suter E., Levit, E.J. (1976). Effectiveness of an international health elective as measured by NBME part II. . Journal of Medical Education, 51(6), 468-472.

Walker, R. J., Campbell, J. A., \& Egede, L. E. (2014). Effective strategies for global health research, training and clinical care: a narrative review. Global Journal of Health Science, 7(2), 119-139. doi:https://dx.doi.org/10.5539/gihs.v7n2p119

Wallace, L., \& Webb, A. (2014). Pre-departure training and the social accountability of International Medical Electives. Education for Health, 27(2), 143-147. doi:10.4103/13576283.143745

Weng, Y. H., Chiou, H. Y., Tu, C. C., Liao, S. T., Bhembe, P. T., Yang, C. Y., \& Chiu, Y. W. (2015). Survey of patient perceptions towards short-term mobile medical aid for those living in a medically underserved area of Swaziland. BMC Health Services Research, 15, 524. doi:10.1186/s12913-015-1186-4

World Health Organization. (2008). Closing the Gap in Generation: Health Equity through Action on Social Determinants of Health. Retrieved from http://www.who.int/social determinants/thecommission/finalreport/en/

Yin, R. (2012). A (Very) Brief Refresher on the Case Study Method. In Applications of Case Study Research (3rd ed.): Sage. 


\section{Appendix A: Guiding Principles for Funding Approval for Global Health Electives}

\section{Guiding Principles for Funding Approval for Global Health Electives:}

1. Safe environment for students

2. Educational value for students with clearly identified learning objectives

3. Pre-departure training and post-trip debriefing with students

4. Preceptors in place who understand and agree with the student's learning objectives

5. Socially responsible and mutually beneficial relationships with host preceptors and partnering institutions based upon a relationship or partnership model

6. Regular feedback to and from partnering institutions concerning the University of Calgary student global health electives

7. Ongoing assessment and evaluation of global health electives

8. Ethical and professional conduct by students and faculty

Eligibility requirements for grant applications by medical students who would like to do a global health elective include:

- the elective must be focused within an underserved or resource-constrained community

- the elective must be a minimum of 4 weeks

- $\quad$ student must submit written confirmation that a preceptor has been arranged (applicable to student directed electives)

The Global Health Student Electives Committee reviews grant applications that meet the aforementioned criteria. If the committee has questions or needs clarification about the application, a committee member may request to meet with the student. Selection of applicants to receive grants are based upon the following considerations:

- $\quad$ student safety - not Extreme Risk

- primary focus of experience on marginalized or underserved community within a population

- realistic/thoughtful/appropriate learning objectives

- rationale for experience

- previous relationship with community either through:

- $\mathrm{U}$ of $\mathrm{C}$ institutional partners

- previous U of C student experiences in location

- student making application has previous relationship in location 


\section{Appendix B: Focus Group Discussion Guide for University of Calgary Medical Students and Residents}

FGD IDNO $\left|\_\right| \_\left|\_\right| \_$Facilitator Initials $\left|\_\right| \_\mid \quad$ Note-taker Initials $\left|\_\right|$

Participant sub-group: (circle): Medical students/Residents

Audio file: $\left|\_\right| \_\mid$

Date

\section{Introduction}

My name is [Moderator] and my colleague [Note-Taker]. I am a graduate student in Community Health Sciences as well as a third-year general surgery resident. My thesis project is to better understand host stakeholder perspectives in relation to medical trainee global health electives by conducting interviews and focus groups at two different host sites in sub-Saharan Africa. The information gathered will be used to further develop the University of Calgary's global health elective program so that they are benefiting all of the people involved including the doctors, nurses, students, patients, family members, and community members at the host sites. Today, I would like to gather some of your perspectives based on your experiences during your global health elective. I also would like to understand what you think may be some of the benefits and harms of global health electives on stakeholders at the host site (i.e. health facility administrators, host physician preceptors, nurses, local trainees, patients and family members, and the community) as well as how you think global health electives could be made more mutually beneficial and equitable.

[Rules of participation in the FGD] Participation in this group discussion is voluntary and you can leave at any time. There is no right or wrong answer, all viewpoints are important. One person speaks at a time and each one of you should be respectful of diverse viewpoints of other people during this discussion. We will keep the identity of participants confidential and anonymous outside of this focus group. If you all accept to participate in this discussion, we will start the audio recording now.

\section{OBTAIN CONSENT-Use consent form}

I would like to ask you some questions with regards to your specific experiences during your global health elective on what you perceive may be benefits and harms of medical trainee global health electives on the host stakeholders. I also would like your input in how you think University of Calgary global health electives could be made more mutually beneficial and equitable.

Opening Question: Can you begin by describing your global health elective experience and in relation to this what do you think are benefits and/or harms of this global health elective on the host stakeholders?

Guiding Questions:

1. Tell me about your experiences working with host physician preceptors.

Probes:

a. Do you think your global health elective benefitted the host physician preceptors?

b. If yes, in what ways?

c. Do you think your global health elective harmed the host physician preceptors?

d. If yes, in what ways?

2. Tell me about your experiences working with other staff, such as nurses or medical officers.

Probes:

e. Do you think your global health elective benefitted other staff?

f. If yes, in what ways? 
g. Do you think your global health elective harmed other staff?

h. If yes, in what ways?

3. Tell me about your experiences working with local medical students or residents.

Probes:

i. Do you think your global health elective benefitted the local trainees?

j. If yes, in what ways?

k. Do you think your global health elective harmed the local trainees?

l. If yes, in what ways?

4. Tell me about your experiences interacting with patients and/or family members.

Probes:

a. Do you think your global health elective benefitted patients and family members?

b. If yes, in what ways?

c. Do you think your global health elective harmed patients and family members?

d. If yes, in what ways?

5. How can the University of Calgary make our global health electives more fair for everyone involved?

6. How can medical trainees improve the experience of host stakeholders during a global health elective?

7. Is there anything you would do to change the process of global health electives?

\section{Closing Questions}

We are now approaching the end of our discussion. Is there anything else anyone would like to add about global health electives that we have not discussed today?

If you had to summarize what we talked about today, what is the most important message that you would like me to learn from our discussion today?

$\checkmark$ Summarize

$\checkmark$ Thank participant

$\checkmark$ Provide extra information and contacts to participants

$\checkmark$ Collect participant demographic details-country travelled to for global health elective 


\section{Appendix C: Interview Guide Introduction}

Facilitator Initials |

Note-taker Initials

Audio file:

Community number:

Date

\section{Introduction}

My name is [Investigator] and my colleague [Local Facilitator]. I am a research student from the University of Calgary in Canada working together with [institution name] to better understand your viewpoints on and experiences with medical students and residents (doctors-in-training) coming from international settings, including from the University of Calgary, for several weeks or months to learn in your hospital. This may be known to you as a global health elective or rotation [check for understanding]. The information gathered will be used to further develop the University of Calgary's global health elective program so that these electives are benefiting all of the people involved including the doctors, nurses, students, patients, family members, and community members here.

[Rules of participation in the interview] Participation in this interview is voluntary and you can leave or decide to end the interview at any time. If there is a question you do not wish to answer, feel free to say so and we will move on to the next question. There is no right or wrong answer, all viewpoints are important. We will keep your identity confidential and anonymous outside of this interview. This discussion will last approximately 30 minutes in length.

Do you have any questions about the consent form? If you have no questions, and if you would like to participate in this discussion, I ask you to please sign the consent form now.

\section{OBTAIN CONSENT-Use consent form}

If you agree with being audio recorded, we will start the audio recording now.

I would like to ask you some questions about your experiences with medical students and residents (doctors-intraining) who travel from international settings (from outside of Africa) to learn medicine and/or surgery in your hospital. In our conversation, I will refer to them as visiting medical trainees [check for understanding]. I am interested to know what your experiences have been and what your thoughts are on this topic area. 


\section{Appendix C1: Interview Guide for Host Physician Preceptors}

Opening Question 1: Can you begin by describing your role here in the hospital?

Opening Question 2: How often have you encountered visiting medical trainees?

Opening Question 3: In what ways have you encountered visiting medical trainees?

Guiding Questions:

1. Can you begin by describing your experiences with visiting medical trainees from foreign countries/international settings at your hospital?Tell me about your experiences supervising visiting medical trainees?

Probes:

a. Tell me how you became involved in this?

b. Why do you continue to be involved in supervising visiting medical trainees?

2. What are your expectations of visiting medical trainees?

a. Probe: How are these expectations met?

b. Probe: How are these expectations not met?

c. Probe: Are visiting medical trainees a pleasure to work with? How?

d. Probe: Are visiting medical trainees difficult to work with? How?

e. Probe: Are visiting medical trainees well-prepared when they come to learn here?

3. What do you observe in how visiting medical trainees interact with other hospital or clinic staff?

a. Probe: Have you observed difficulties? Can you give an example.

b. Probe:Have you observed positive interactions? Can you give an example.

c. Probe: Have other staff, for example, nurses or medical officers, discussed visiting medical trainees with you? (what do they tell you about these visiting medical trainees?)

4. What do you observe in how visiting medical trainees interact with local students and trainees?

a. Probe: Have you observed difficulties? Can you give an example.

b. Probe:Have you observed positive interactions? Can you give an example.

5. What do you observe in how visiting medical trainees interact with patients or their families?

a. Probe: Have you observed difficulties? Can you give an example.

b. Probe:Have you observed positive interactions? Can you give an example.

6. What do you think are benefits in having visting medical trainees come to learn at your hospital?

a. Probe: Has it created any opportunities for you?

b. Probe: Has it improved your own practice in any way?

7. What do you think are harms in having visiting medical trainees come to learn in your hospital?

a. Probe: are there any negative issues in having visiting medical trainees come to learn in your hospital? 
b. Probe: are visiting medical trainees an obligation?

c. Probe: Some research suggests language and cultural barriers during global health electives may harm patient care. Do you find this to be an issue? (can you give an example from your own experiences)

d. Probe: Another question identified in some research on global health electives is that visiting medical trainees may take learning opportunities away from local learners. What are your thoughts and experiences with this?

e. Probe: Are visiting medical trainees disruptive in any way?

8. What can the University of Calgary do to improve your experience and this hospital's experience when our medical trainees come to learn here?

a. What can our medical trainees do when they come to learn here to improve your experience and this hospital's experience?

b. Does it make any difference to your institution to have visiting medical trainees come with a foreign preceptor? Does that provide any positive or negative effect?

9. How can the University of Calgary make our global health electives more fair for everyone involved?

10. Is there anything you would like to do to change the process of visiting medical trainees coming to your hospital?

\section{Closing Questions}

We are now approaching the end of our discussion. Is there anything else you would like to add about visiting medical trainees coming here to learn that we have not yet talked about?

If you had to summarize what we talked about today, what is the most important message that you would like me to learn from our discussion today?

$\checkmark$ Summarize

$\checkmark$ Thank participant

$\checkmark$ Provide extra information and contacts to participants

Collect participant demographic details-role/occupation, where they trained, how many students they supervise per month (both visiting medical trainees and local trainees), how many years in practice 


\section{Appendix C2: Interview Guide for Hospital/Health Facility Administrator}

Opening Question 1: Can you begin by describing your role here in the hospital?

Opening Question 2: How often have you encountered visiting medical trainees?

Opening Question 3: In what ways have you encountered visiting medical trainees?

Guiding Questions:

1. Can you begin by describing your experiences with visiting medical trainees from foreign countries/international settings at your hospital?Tell me about your involvement in having visiting medical trainees coming to learn at your hospital?

Probes:

a. Have you always been involved with the process of having visiting medical trainees coming to your hospital?

b. Tell me how you became involved in this?

c. What details or decisions are you involved in when visiting medical trainees come to your hospital?

d. Why do you continue to be involved in this process?

e. What makes it possible for this hospital to allow visiting medical trainees to come here to learn?

2. What are your expectations of visiting medical trainees?

a. Probe: How are these expectations met?

b. Probe: How are these expectations not met?

c. Probe: Are visiting medical trainees a pleasure to work with? How?

d. Probe: Are visiting medical trainees difficult to work with? How?

e. Are visiting medical trainees well-prepared when they come here to learn?

3. How do visiting medical trainees interact with other staff in the hospital/clinic?

a. Probe: Have you observed difficulties? Can you give an example.

b. Probe:Have you observed positive interactions? Can you give an example.

c. Probe: Have other staff, for example, nurses or medical officers, discussed visiting medical trainees with you? (what do they tell you about these visiting medical trainees?)

4. Do visiting medical trainees interact with local trainees?

a. Probe: If yes, how do visiting medical trainees interact with local trainees?

b. Probe: Have you observed difficulties? Can you give an example.

c. Probe:Have you observed positive interactions? Can you give an example.

5. How do visiting medical trainees interact with patients or their families? 
a. Probe: Have you observed difficulties? Can you give an example.

b. Probe:Have you observed positive interactions? Can you give an example.

6. What do you think are benefits in having medical students and residents come to learn in your hospital?

a. Probe: Has it created any opportunities for you?

b. Probe: Has it improved the hospital in any way?

7. What do you think are harms in having medical students and residents come to learn in your hospital?

a. Probe: are there any negative things in allowing visiting medical trainees to come learn in your hospital?

b. Probe: are visiting medical trainees an obligation?

c. Probe: Some research suggests language and cultural barriers during global health electives may harm patient care. Do you find this to be an issue? (can you give an example from your own experiences)

d. Probe: Another question identified in some research on global health electives is that visiting medical trainees may take learning opportunities away from local learners. What are your thoughts and experiences with this?

e. Probe: Are visiting medical trainees disruptive in any way?

8. What can the University of Calgary do to improve your experience and this hospital's experience when our medical trainees come to learn here?

a. What can our medical trainees do when they come to learn here to improve your experience and this hospital's experience?

b. Does it make any difference to your institution to have visiting medical trainees come with a foreign preceptor? Does that provide any positive or negative effect?

9. How can the University of Calgary make our global health electives more fair for everyone involved?

10. Is there anything you would like to do to change the process of visiting medical trainees coming to your hospital?

\section{Closing Questions}

We are now approaching the end of our discussion. Is there anything else anyone would like to add about visiting medical trainees coming here to learn that we have not yet talked about?

If you had to summarize what we talked about today, what is the most important message that you would like me to learn from our discussion today?
$\checkmark$ Summarize
$\checkmark$ Thank participant
$\checkmark$ Provide extra information and contacts to participants
$\checkmark$ Collect participant demographic details-role/occupation, place of training 


\section{Appendix C3: Interview Guide for Community Liaison Member}

Opening Question 1: Can you begin by describing your role or position?

Opening Question 2: How often have you encountered visiting medical trainees?

Opening Question 3: In what ways have you encountered visiting medical trainees?

Guiding Questions:

1. What do you think about visiting medical trainees coming to this hospital and to this community to learn?

a. Probe: Describe an experience.

2. What are your expectations of visiting medical trainees?

a. probe: are these expectations met/not met?

b. How do you think these visiting medical trainees should be prepared before coming to your community?

3. What do you think are benefits in allowing visiting medical trainees to come learn in this hospital?

a. Probe: Has it improved the community in any way?

4. What do you think are harms in having visiting medical trainees come to learn in this community?

a. Probe: Are visiting medical trainees disruptive to the community in any way?

b. Probe: Some research suggests language and cultural barriers during global health electives may harm patient care. What are your thoughts on this? (can you give an example from your own experiences)

5. What can the University of Calgary do to improve your experience and this community's experience when our medical trainees come to learn here?

a. What can our medical trainees do when they come to learn here to improve the community's experience?

6. How can the University of Calgary make our global health electives more fair for everyone involved?

Closing Question: Is there anything else that you would like to share or discuss regarding global health electives?

If you had to summarize what we talked about today, what is the most important message that you would like me to learn from our discussion today? 


\section{Appendix C4: Interview Guide for Nurses}

Opening Question 1: Can you begin by describing your role or position?

Opening Question 2: How often have you encountered visiting medical trainees?

Opening Question 3: In what ways have you encountered visiting medical trainees?

\section{Guiding Questions:}

1. Can you begin by describing your experiences with visiting medical trainees from foreign countries/international settings at your hospital?

2. Tell me about your experiences working with visiting medical trainees?

Probes:

a. How do visiting medical trainees affect your clinical duties?

b. Probe: Are visiting medical trainees a pleasure to work with? How?

c. Probe: Are visiting medical trainees difficult to work with? How?

3. What are your expectations of visiting medical trainees?

a. Probe: How are these expectations met?

b. Probe: How are these expectations not met?

c. Are visiting medical trainees well-prepared when they come here to learn?

4. How do visiting medical trainees interact with other staff in the hospital/clinic?

a. Probe: Have you observed difficulties? Can you give an example.

b. Probe:Have you observed positive interactions? Can you give an example.

c. Probe: Have other nurses discussed visiting medical trainees with you? (what do they tell you about these visiting medical trainees?)

5. Do visiting medical trainees interact with local trainees?

a. Probe: Have you observed difficulties? Can you give an example.

b. Probe:Have you observed positive interactions? Can you give an example.

6. How do visiting medical trainees interact with patients or their families?

a. Probe: Have you observed difficulties? Can you give an example.

b. Probe:Have you observed positive interactions? Can you give an example.

7. What do you think are benefits in allowing medical students and residents to come learn in your hospital?

a. Probe: Has it created any opportunities for you?

b. Probe: Has it improved your own learning in any way?

8. What do you think are harms in allowing medical students and residents to come learn in your hospital? 
a. Probe: are there any negative things in allowing visiting medical trainees to come learn in your hospital?

b. Probe: Some research suggests language and cultural barriers during global health electives may harm patient care. Do you find this to be an issue? (can you give an example from your own experiences)

c. Probe: Another question identified in some research on global health electives is that visiting medical trainees may use limited resources available in the hospital and thereby drain local resources though other research suggests visiting medical trainees may be helpful in assisting with patient care. What are your thoughts and experiences with this?

d. Another question identified in some research on global health electives is that visiting medical trainees may take learning opportunities away from local learners. What are your thoughts and experiences with this?

e. Probe: Are visiting medical trainees disruptive in any way?

9. What can the University of Calgary do to improve your experience and this hospital's experience when our visiting medical trainees come to learn here?

a. What can our medical trainees do when they come to learn here to improve your experience and this hospital's experience?

10. How can the University of Calgary make our global health electives more fair for everyone involved?

11. Is there anything you would like to do to change the process of visiting medical trainees coming to your hospital?

\section{Closing Questions}

We are now approaching the end of our discussion. Is there anything else you would like to add about visiting medical trainees coming here to learn that we have not yet talked about?

If you had to summarize what we talked about today, what is the most important message that you would like me to learn from our discussion today?
$\checkmark$ Summarize
$\checkmark$ Thank participant
$\checkmark$ Provide extra information and contacts to participants
$\checkmark$ Collect participant demographic details-role/occupation, years in practice 


\title{
Appendix D: Focus Group Guide Generic Introduction/Ground Rules
}

\author{
FGD IDNO $\left|\_\right| \_\mid \_$Facilitator Initials $\left|\_\right| \_$Note-taker Initials $\left|\_\right|$
}

Assistant Initials

Participant sub-group: (circle): Medical Officers/Medical students/Residents/Patients/Family Members

Audio file:

Community number:

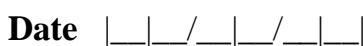

\section{Introduction}

My name is [Moderator] and my colleagues [Note-Taker] and [Assistant]. Adriena De Visser is a research student from the University of Calgary in Canada working together with [institution name] to better understand your viewpoints on and experiences with medical students and residents (doctors-in-training) coming from foreign countries, including from the University of Calgary, for several weeks or months to learn in your hospital. This is known as a global health elective or rotation. The information gathered will be used to further develop the University of Calgary's global health elective program so that they are benefiting all of the people involved including the doctors, nurses, students, patients, family members, and community members here.

[Rules of participation in the FGD] Participation in this group discussion is voluntary and you can leave at any time. There is no right or wrong answer, all viewpoints are important. One person speaks at a time and each one of you should be respectful of diverse viewpoints of other people during this discussion. We will keep the identity of participants confidential and anonymous outside of this focus group. If you all accept to participate in this discussion, we will start the audio recording now.

\section{OBTAIN CONSENT-Use consent form}

I would like to ask you some questions about your experiences with medical students and residents (doctors-intraining) who travel from international settings (from outside of Africa) to learn medicine and/or surgery in your hospital. In our conversation, I will refer to them as visiting medical trainees [check for understanding]. I am interested to know what your experiences have been and what your thoughts are on this topic area. 


\section{Appendix D1: Focus Group Discussion Guide for Medical Officers/Registrars}

Opening Question 1: Can you begin by describing your role here in the hospital?

Opening Question 2: How often have you encountered visiting medical trainees?

Opening Question 3: In what ways have you encountered visiting medical trainees?

Guiding Questions:

1. Can you begin by describing your experiences with visiting medical trainees from foreign countries/international settings at your hospital?Tell me about your experiences working with visiting medical trainees?

Probes:

a.Tell me how you became involved in this?

2. What are your expectations of visiting medical trainees?

a. Probe: How are these expectations met?

b. Probe: How are these expectations not met?

c. Probe: Are visiting medical trainees a pleasure to work with? How?

d. Probe: Are visiting medical trainees difficult to work with? How?

e. Probe: Are visiting medical trainees well-prepared when they come to learn here?

3. How do visiting medical trainees interact with other staff in the hospital/clinic?

a. Probe: Have you observed difficulties? Can you give an example.

b. Probe:Have you observed positive interactions? Can you give an example.

c. Probe: Have other staff, for example, nurses or other medical officers, discussed visiting medical trainees with you? (what do they tell you about these visiting medical trainees?)

4. Do visiting medical trainees interact with local trainees?

a. Probe: Have you observed difficulties? Can you give an example.

b. Probe:Have you observed positive interactions? Can you give an example.

5. How do visiting medical trainees interact with patients or their families?

a. Probe: Have you observed difficulties? Can you give an example.

b. Probe:Have you observed positive interactions? Can you give an example.

6. What do you think are benefits in allowing visting medical trainees to come learn in your hospital?

a. Probe: Has it created any opportunities for you?

b. Probe: Has it improved your own learning in any way?

7. What do you think are harms in allowing visiting medical trainees to come learn in your hospital?

a. Probe: are there any negative things in allowing visiting medical trainees to come learn in your hospital? 
b. Probe: Some research suggests language and cultural barriers during global health electives may harm patient care. Do you find this to be an issue? (can you give an example from your own experiences)

c. Probe:Another question identified in some research on global health electives is that visiting medical trainees may take learning opportunities away from local learners. What are your thoughts and experiences with this?

d. Probe: Are visiting medical trainees disruptive in any way?

8. What can the University of Calgary do to improve your experience and this hospital's experience when our medical trainees come to learn here?

a. What can our medical trainees do when they come to learn here to improve your experience and this hospital's experience?

9. How can the University of Calgary make our global health electives more fair for everyone involved?

10. Is there anything you would like to do to change the process of visiting medical trainees coming to your hospital?

\section{Closing Questions}

We are now approaching the end of our discussion. Is there anything else anyone would like to add about visiting medical students and residents coming here to learn that we have not yet talked about?

If you had to summarize what we talked about today, what is the most important thing that you would like me to learn from our discussion today?

$\checkmark$ Summarize

$\checkmark$ Thank participant

$\checkmark$ Provide extra information and contacts to participants

Collect participant demographic details-role/occupation, years in practice 


\section{Appendix D2: Focus Group Interview Guide for Local Residents, Interns, and Medical Students}

Opening Question: Can you begin by describing your experiences with visiting medical trainees from foreign countries/international settings at your hospital?

Guiding Questions:

2. Tell me about your experiences working with visiting medical trainees?

Probes:

a. Can you give me an example?

b. Do visiting medical trainees change your own training in any way?

c. How much do you interact with visiting medical trainees?

d. How do you interact with visiting medical trainees?

3. What are your expectations of visiting medical trainees?

a. Probe: How are these expectations met?

b. Probe: How are these expectations not met?

c. Probe: Are visiting medical trainees a pleasure to work with? How?

d. Probe: Are visiting medical trainees difficult to work with? How?

e. Probe: Are visiting medical trainees well-prepared when they come to learn here?

4. How do visiting medical trainees interact with other staff in the hospital/clinic?

a. Probe: Have you observed difficulties? Can you give an example.

b. Probe:Have you observed positive interactions? Can you give an example.

c. Probe: Have other staff, for example, nurses or other medical officers, discussed visiting medical trainees with you? (what do they tell you about these visiting medical trainees?)

5. How do visiting medical trainees interact with patients or their families?

a. Probe: Have you observed difficulties? Can you give an example.

b. Probe:Have you observed positive interactions? Can you give an example.

6. What do you think are benefits in allowing visting medical trainees to come learn in your hospital?

a. Probe: Has it created any opportunities for you?

b. Probe: Has it improved your own learning in any way?

7. What do you think are harms in allowing visiting medical trainees to come learn in your hospital?

a. Probe: are there any negative things in allowing visiting medical trainees to come learn in your hospital?

b. Probe: Some research suggests language and cultural barriers during global health electives may harm patient care. Do you find this to be an issue? (can you give an example from your own experiences) 
c. Probe: Another question identified in some research on global health electives is that visiting medical trainees may take learning opportunities away from local learners. What are your thoughts and experiences with this?

d. Probe: Are visiting medical trainees disruptive in any way?

8. What can the University of Calgary do to improve your experience when our medical trainees come to learn here?

a. What can our medical trainees do when they come to learn here to improve your experience?

9. How can the University of Calgary make our global health electives more fair for everyone involved?

10. Is there anything you would like to do to change the process of visiting medical trainees coming to your hospital?

\section{Closing Questions}

We are now approaching the end of our discussion. Is there anything else anyone would like to add about visiting medical trainees coming here to learn that we have not yet talked about?

If you had to summarize what we talked about today, what is the most important message that you would like me to learn from our discussion today?

$\checkmark$ Summarize

$\checkmark$ Thank participant

$\checkmark$ Provide extra information and contacts to participants

Collect participant demographic details-role/occupation, year of training 


\section{Appendix D3: Focus Group Discussion Guide for Patients and Family Members}

Opening Question 1: As a patient or family member, have you met any visiting medical trainees? (make sure to clarify who they are talking about)

Guiding Questions:

1. What was that like?

2. Tell me about your experiences interacting with visiting medical trainees?

Probes:

a. How much do you talk with the visiting medical trainees?

b. Can you give me an example of how they interacted with you.

c. What have the visiting medical trainees done for you as a patient or family member?

3. What are your expectations of visiting medical student and residents?

a. Probe: Are these expectations met or not met?

4. What do you think are benefits in having visiting medical trainees coming to learn in this hospital?

a. Probe: Has it improved your experience while you are here in any way, either as a patient or a family member?

b. Can you describe how it has improved your experience?

5. What do you think are harms in having visiting medical trainees coming to learn in this hospital?

a. Probe: are there any negative things in allowing medical students and residents to come learn in this hospital?

b. Probe: Some research suggests language and cultural barriers during global health electives may harm patient care. Do you find this to be an issue? (can you give an example from your own experiences)

c. Probe: Another question identified in some research on global health electives is that visiting medical trainees may use limited resources available in the hospital and thereby drain local resources though other research suggests visiting medical trainees may be helpful in assisting with patient care. What are your thoughts and experiences with this?

6. How can the University of Calgary make our global health electives more fair for everyone involved?

7. What can University of Calgary medical trainees do to improve your experiences when they come to learn in this hospital?

8. Is there anything you would like to change when visiting medical trainees come to this hospital to learn and to help take care of you?

\section{Closing Question}

We are now approaching the end of our discussion. Is there anything else anyone would like to add about visiting medical students and residents coming here to learn that we have not yet talked about?

If you had to summarize what we talked about today, what is the most important message that you would 
like me to learn from our discussion today?

$\checkmark$ Summarize

$\checkmark$ Thank participants

$\checkmark$ Provide extra information and contacts to participants

Collect participant demographic details-role, length of stay in hospital (outpatient vs. inpatient) 